\title{
Mapping stellar content to dark matter halos using galaxy clustering and galaxy-galaxy lensing in the SDSS DR7
}

\author{
Ying $\mathrm{Zu}^{\star}$ and Rachel Mandelbaum \\ McWilliams Center for Cosmology, Department of Physics, Carnegie Mellon University, 5000 Forbes Avenue, Pittsburgh, PA 15213, USA
}

20 August 2018

\begin{abstract}
The mapping between the distributions of the observed galaxy stellar mass and the underlying dark matter halos provides the crucial link from theories of large-scale structure formation to interpreting the complex phenomena of galaxy formation and evolution. We develop a novel statistical method, based on the Halo Occupation Distribution model (HOD), to solve for this mapping by jointly fitting the galaxy clustering and the galaxy-galaxy lensing measured from the Sloan Digital Sky Survey (SDSS). The method, called the iHOD model, extracts maximum information from the survey by including $\sim 80 \%$ more galaxies than the traditional HOD methods, and takes into account the incompleteness of the stellar mass samples in a statistically consistent manner. The derived stellar-to-halo mass relation not only explains the clustering and lensing of SDSS galaxies over almost four decades in stellar mass, but also successfully predicts the stellar mass functions observed in SDSS. Due to its capability of modelling significantly more galaxies, the $i \mathrm{HOD}$ is able to break the degeneracy between the logarithmic scatter in the stellar mass at fixed halo mass and the slope of the stellar-to-halo mass relation at high mass end, without the need to assume a strong prior on the scatter and/or use the stellar mass function as an input. We detect a decline of the scatter with halo mass, from $0.22_{-0.01}^{+0.02}$ dex at below $10^{12} h^{-1} M_{\odot}$ to $0.18 \pm 0.01$ dex at $10^{14} h^{-1} M_{\odot}$. The model also enables stringent constraints on the satellite stellar mass functions at fixed halo mass, predicting a departure from the Schechter functional form in high mass halos. The $i \mathrm{HOD}$ model can be easily applied to existing and future spectroscopic datasets, greatly improving the statistical constraint on the stellar-to-halo mass relation compared to the traditional HOD methods within the same survey.
\end{abstract}

Key words: cosmology: observations - cosmology: large-scale structure of Universe galaxies: luminosity function, mass function — gravitational lensing: weak - methods: statistical

\section{INTRODUCTION}

The distribution of galaxy stellar mass in the present-day Universe provides important clues to answering fundamental questions concerning the cosmic assembly of baryons in the $\Lambda$-cold dark matter $(\Lambda \mathrm{CDM})$ paradigm (Fukugita et al. 1998, Kereš et al. 2005. Faucher-Giguère et al. 2011, Davé et al. 2012). In particular, what fraction of baryons are condensed into stars as opposed to spreading out in the form of gas and dust in and outside of galaxies (Cen \& Ostriker 1999, McGaugh et al. 2010, Putman et al. 2012, Shull et al.2012)? Among those locked in stars, how much stellar mass is stored within the central galaxies of the dark matter halos (De Lucia \& Blaizot 2007, von der Linden et al.2007), and how is the rest distributed among the satellite galaxies within their larger host halos (Hansen et al. 2009, Yang et al. 2009, Leauthaud et al.2012a)?

* E-mail: yzu@cmu.edu
In this paper we develop a novel statistical approach within the Halo Occupation Distribution (HOD; Jing et al. 1998, Ma \& Fry 2000, Peacock \& Smith 2000, Seljak 2000|, Yang et al. 2003; Scoccimarro et al. 2001, Cooray \& Sheth 2002; Berlind \& Weinberg 2002, Guzik \& Seljak 2002, Zheng et al. 2005. Mandelbaum et al. 2006, van den Bosch et al. 2007) framework, to solve for the mapping between the stellar mass content and the dark matter halos using the spatial clustering and the weak gravitational lensing of the Sloan Digital Sky Survey (SDSS; York et al. 2000) spectroscopic sample galaxies. The inferred mapping not only explains the galaxy auto-correlation function (i.e., clustering) and the galaxy-matter cross-correlation (i.e., lensing) successfully, but also correctly predicts the observed stellar mass function (SMF), placing strong constraints on the physics that governs the formation and distribution of galaxies within halos.

The most comprehensive way to link the galaxy properties to the dark matter halos is to directly model the expected phys- 
ical processes involved in the formation and evolution of stars and gas (along with metals and dust), by either running hydrodynamic simulations (Hernquist \& Katz 1989, Katz et al. 1996, Norman \& Bryan 1999, Teyssier 2002, O'Shea et al. 2004, Springel et al. 2005, Di Matteo et al. 2005. Oppenheimer \& Davé 2006. Booth \& Schaye 2009, Vogelsberger et al. 2013) or semi-analytical models (SAMs) along the halo merger trees of N-body simulations (Baugh 2006; Somerville et al. 2008; Kang et al. 2005, Bower et al. 2006, De Lucia et al. 2006). The primary advantage of the latter approach is that it is computationally much less expensive compared to the hydrodynamic simulations, albeit with a large number of free parameters. However, even within the hydrodynamic simulations some of the key processes, like the star formation from the collapse of molecular clouds, the stellar feedback from supernovae explosions and galactic winds, and the impact of the active galactic nuclei (i.e., the AGN feedback), are happening on scales well below the resolution limit, so the treatment of the physics is often at the "subgrid" level, i.e., put in by hand using empirical scaling relations (see Benson 2010 for a review). Both the hydrodynamic simulations and the SAMs nonetheless have enjoyed great success over the past decade, reproducing a wide range of the observed galaxy properties (see Oppenheimer \& Davé 2008 Khandai et al. 2014, and Vogelsberger et al. 2014 for the latest hydrodynamic simulations and see Guo et al. 2011 for the recent development in SAM), though still with several possible areas of improvement. Most importantly, because the simulated outputs are directly tied to the prescribed subgrid physics and its parameters, by confronting the predictions of this "forward modelling" approach with new observations, we can constantly furnish our understanding of the galaxy formation physics in a cosmological context, especially the baryonic feedback mechanisms that help sculpt the galaxy SMF (Fontanot et al. 2009). However, the computational complexities of hydrodynamic simulations and the SAM degeneracies make it difficult to come to definite conclusions about galaxy formation theories in certain cases.

Alternatively, one can focus on the statistical link between just the stellar content and the dark matter halos, assuming that the enormous diversity in the individual galaxy assembly histories inside halos of the same mass would reduce to a stochastic scatter about the mean stellar-to-halo mass relation (SHMR) by virtue of the central limit theorem. The great success of the HOD framework in explaining the clustering and lensing of galaxies, and their dependencies on galaxy properties like colour, luminosity, and stellar mass at different redshifts (e.g., Zehavi et al. 2011; Parejko et al. 2013 , Guo et al. 2014), further validates this assumption about the statistical simplicity of the SHMR. For a given cosmology, the HOD formalism describes the relationship between the stellar and dark matter in terms of $\left\langle N_{g}\left(M_{h}\right)\right\rangle$, the average number of galaxies of a given type (e.g., central or satellite) within a halo of virial mass $M_{h}$, along with the spatial and velocity distributions of galaxies within that halo (see Yang et al.2003 for a closely related approach, the conditional luminosity function). Furthermore, the HOD is potentially a powerful tool to constrain cosmology (Yoo et al. 2006. Zheng \& Weinberg 2007, van den Bosch et al. 2013, Cacciato et al. 2013. More et al. 2014), by exploiting the average bias vs. mass relation of the dark matter halos revealed by the galaxies they contain. The standard HOD modelling is restricted to individual volumelimited samples, each defined by a stringent combination of stellar mass and redshift cuts, which leaves many observed galaxies unused. Moreover, the corresponding HOD parameters are inferred separately for each sample, therefore the SHMR is constrained at a disjunct set of loci where the mean stellar masses of the samples landed.

Recently, Leauthaud et al. (2011; hereafter L11) proposed a new HOD framework by further parameterizing $\left\langle N_{g}\left(M_{h}\right)\right\rangle$ as continuous functions of the threshold stellar mass $M_{*}$, i.e., $\left\langle N_{g}\left(>M_{*} \mid M_{h}\right)\right\rangle$. In particular, the mean SHMR of central galaxies and its scatter fully specify the expected number of central galaxies of any stellar mass at fixed halo mass, whereas the number of satellite galaxies above a certain stellar mass scales with halo mass in a way that is also halo mass-dependent. The advantage of this new framework lies in its unique capability to derive the connection between galaxies and halos using multiple probes simultaneously within a single global HOD model. Leauthaud et al. (2012b, hereafter L12) demonstrated the efficacy of the L11 model by inferring the SHMR across the entire observed stellar mass range, using the combination of the SMF, angular galaxy clustering, and galaxy-galaxy ( $\mathrm{g}$-g) lensing of samples above some critical stellar mass limit (corresponding to high completeness) in the COSMOS survey. However, similar to the standard HOD technique, the L11 model requires volume completeness in the stellar mass samples, thereby losing a lot of data in an intrinsically flux-limited survey. Our approach is based on the L11 global HOD framework, and (like in L11) can jointly fit the galaxy clustering and g-g lensing signals of galaxy samples above some critical stellar mass limit, but (unlike in L11) can take into account the incompleteness in a statistically self-consistent way. As a result of the added flexibility of our model, we are able to include significantly more SDSS galaxies at both lower stellar mass and higher redshift, equivalent to almost doubling the survey volume.

Meanwhile, Moster et al. (2010, hereafter M10) and other groups (e.g., Kravtsov et al. 2004, Vale \& Ostriker 2004, Conroy et al. 2006, Shankar et al. 2006, Behroozi et al. 2010, Guo et al. 2010) adopted the so-called "sub-halo abundance matching" (SHAM) technique to assign stellar masses or luminosities to individual dark matter halos (including both main and subhalos) in the N-body simulations. In its simplest form, the SHAM method assumes a monotonic relation between the SMF estimated from galaxy surveys and the halo mass function predicted by the $\Lambda \mathrm{CDM}$. Instead of using the subhalo mass at the current epoch, most SHAM studies employed the "infall mass", i.e., the mass of the subhalo before its accretion onto the main halo, and instead of a monotonic relation they either assumed some fixed scatter or drew the scatter from external priors. Using the "infall mass" as a proxy for the stellar mass, they found surprisingly good agreement between the predicted and the observed clustering and lensing statistics. The underlying assumption behind SHAM is that the satellite galaxies that live in subhalos were central galaxies of their own halos before accretion. After becoming satellites, the dark matter masses of their subhalos were reduced due to the combined effect of tidal stripping and dynamical friction, whereas their stellar masses in the centre of those subhalos were much less affected and are thus more closely tied to the infall masses (Reddick et al. 2013. van den Bosch et al. 2005). Hearin \& Watson (2013) later extended this assumption to further relate the halo formation time to the colour of each galaxy to interpret the colour dependence of the clustering and lensing statistics (also see Kulier \& Ostriker 2015. for an interesting proposal of a two-parameter abundance matching scheme). The key distinction between the SHAM and the HOD models is that, by using the infall mass the SHAM method evades the need to parameterize the HOD of the satellite galaxies (but see Guo et al.2011. on potential numerical issues in tracking subhalos), which is usually regarded as a nuance in traditional HOD mod- 
elling, at the expense of assuming the same SHMRs for the central and the satellite galaxies. However, as we will demonstrate in this study, the capability of our modified HOD model to maximally extract the information from data enables us to place tight constraint on the satellite HOD as well, thus shedding new insight into the formation and evolution of the satellite galaxies without imposing the central SHMR on the satellites. Moreover, the model breaks the degeneracy between the scatter and the slope of the SHMR, selfconsistently deriving the scatter without the need to assume fixed values or external priors.

The paper is organised as follows. Section 2 describes the SDSS data, including the large-scale structure galaxy catalogue and the matched stellar mass catalogue, and the two sets of galaxy samples selected for our analyses. In Section 3, we describe the methods we use to measure galaxy clustering and g-g lensing for these samples. Section 4 introduces our new variant of the HOD model that we use to interpret the clustering and lensing signals, and Section 5 describes the method to theoretically predict these signals using our HOD model. In Section 6 we present the results of our constraint from a Bayesian framework via the new HOD modelling technique. We examine the SMF, the SHMR of the central galaxies, and the conditional SMFs of the satellites predicted by our best-fit model in Section 7 We summarise our main findings and discuss future applications of the new HOD model in Section 8

Throughout this paper, we assume a $\Lambda \mathrm{CDM}$ cosmology with $\left(\Omega_{m}, \Omega_{\Lambda}, \sigma_{8}, h\right)=(0.26,0.74,0.77,0.72)$. All the length and mass units in this paper are scaled as if the Hubble constant were $100 \mathrm{~km} \mathrm{~s}^{-1} \mathrm{Mpc}^{-1}$. In particular, all the separations are co-moving distances in units of either $h^{-1} \mathrm{kpc}$ or $h^{-1} \mathrm{Mpc}$, and the stellar mass and halo mass are in units of $h^{-2} M_{\odot}$ and $h^{-1} M_{\odot}$, respectively. The halo mass is defined by $M_{h} \equiv M_{200 m}=200 \bar{\rho}_{m}(4 \pi / 3) r_{200 m}^{3}$, where $r_{200 m}$ is the corresponding halo radius within which the average density of the enclosed mass is 200 times the mean matter density of the Universe, $\bar{\rho}_{m}$. For the sake of simplicity, $\ln x=\log _{e} x$ is used for the natural logarithm, and $\lg x=\log _{10} x$ is used for the base-10 logarithm.

\section{DESCRIPTION OF THE DATA}

Here we describe the spectroscopic data used to define the clustering and the lens samples, and the imaging data used to define the source samples for $\mathrm{g}-\mathrm{g}$ lensing.

\subsection{SDSS Main Galaxy Sample and NYU-VAGC}

This study is based on the final data release of the SDSS (DR7; Abazajian et al. 2009), which contains the completed data set of the SDSS-I and the SDSS-II. The survey imaged a quarter of the sky using a drift-scan camera (Gunn et al. 1998) in five photometric bandpasses $(u, g, r, i, z$; Fukugita et al. 1996) to a limiting magnitude of $\simeq 22.5$ in the $r$ band. The imaging data were photometrically and astrometrically calibrated (Padmanabhan et al. 2008), and from this imaging data targets were selected for spectroscopic follow-ups with a fibre-fed double spectrograph (Gunn et al. 2006). One of the spectroscopic products is "the main galaxy sample" (MGS; Strauss et al. 2002) that we use in this study as both the tracers of stellar mass clustering and the lenses of background sources. In particular, we obtain the MGS data from the dr72 large-scale structure sample bright 0 of the "New York University Value Added Catalogue" (NYU-VAGC), constructed as described in Blanton et al. (2005). The bright 0 sample includes galaxies with $10<m_{r}<17.6$, where $m_{r}$ is the $r$-band Petrosian apparent magnitude, corrected for Galactic extinction. We choose a more relaxed bright limit than the commonly used safe 0 sample $\left(14.5<m_{r}<17.6\right)$ to allow higher completeness on the high stellar mass end, where constraint on the mapping between galaxies and halos is particularly lacking.

Due to the finite size of the fibre plugs, no two targets on the same plate can be closer than $55^{\prime \prime}$, resulting in $\sim 7 \%$ of the MGS galaxies with unknown redshifts. A simple remedy for these fibre collisions, as used in the bright 0 sample, is to assign these galaxies the redshifts of their nearest neighbours on the sky, thus exactly preserving the angular clustering signal. For our purpose of measuring the projected correlation $w_{p}$ at a given redshift $z$, it would overestimate the clustering signal below the projected physical scale corresponding to the fibre size at that redshift, $r_{p}^{\mathrm{fb}}(z)$, by including physically distant pairs of galaxies that happen to align within $55^{\prime \prime}$ on the sky. Above $r_{p}^{\mathrm{fb}}(z)$, however, this "nearest-neighbour" redshift assignment scheme recovers the underlying $w_{p}$ remarkably well (Zehavi et al. 2005). We thus limit our $w_{p}$ measurement to those scales above. We do not consider other more sophisticated corrections that would potentially recover the signals below the fibre radius (Guo et al.2012), as $r_{p}^{\mathrm{fb}}$ is always below $0.17 h^{-1} \mathrm{Mpc}$ across the redshift range of our samples $\left(z_{\max }=0.30\right)$. Furthermore, even at the highest redshifts where $r_{p}^{\mathrm{fb}}$ is relatively large, the samples are progressively dominated by more massive galaxies that have a larger correlation length, so the one-halo term is still well resolved in the clustering measurement above $r_{p}^{\mathrm{fb}}$. For the $\mathrm{g}$ $\mathrm{g}$ lensing measurement, this correction using the nearest neighbour slightly blurs the signals around those "collided" lenses in the transverse direction because of the inaccurate conversion from angles to physical separations. This blurring effect can be safely ignored in our analysis as it does not reduce the overall amplitude of the average lensing signal.

In addition to the angular positions and redshifts of the galaxies, the NYU-VAGC also calculated k-corrections for all the MGS galaxies using templates based on stellar population synthesis (SPS) models, while providing approximate stellar mass estimates and star formation histories for all the bright 0 galaxies (Blanton \& Berlind 2007). Comparison with the stellar mass estimates from Kauffmann et al. (2003) shows a scatter of only 0.1 dex and mean biases of less than 0.2 dex depending on stellar mass. This tight relationship, as we show later in Section 2.2. provides crucial information for estimating stellar masses for galaxies that do not have valid entries in the MPA/JHU stellar mass catalogue (an updated version of the Kauffmann et al. 2003 catalogue) that we adopted.

To minimise any potential artefacts in the measurements due to irregular survey geometry and areas with low spectroscopic coverage, we use data exclusively within the contiguous area in the North Galactic Cap and from sectors (each defined by a region covered by a unique set of plates) where the angular completeness is greater than 0.8 . The final sample used for the galaxy clustering analysis includes 513,150 galaxies over a sky area of 6395.49 $\mathrm{deg}^{2}$. A further 5 per cent of the area is eliminated for the lensing analysis, due to the absence of source galaxies in that area (for example, because of poor imaging quality or more conservative masking around bright stars). 


\subsection{Stellar Mass Estimate}

We employ the stellar mass estimates from the latest MPA/JHU value-added galaxy catalogue ${ }^{1}$ The stellar masses were estimated based on fits to the SDSS photometry following the philosophy of Kauffmann et al. (2003) and Salim et al. (2007), and assuming the Chabrier (Chabrier 2003) initial mass function (IMF) and the Bruzual \& Charlot (2003) SPS model. The estimation is very similar to the spectroscopic fits as described in Kauffmann et al. (2003), but instead of spectral features like the D4000 index and the $\mathrm{H} \delta$ absorption lines, the broad-band photometry (after correction for emission lines) is used for the fits. The key difference between the photometry-based estimates and the spectroscopic ones is that the stellar mass-to-light ratio $\left(M_{*} / L\right)$ estimated from spectroscopic features is only representative for the region sampled by the fibre, and a constant $M_{*} / L$ must then be assumed to extrapolate to a total stellar mass. To avoid extrapolation, we employ the total mass estimates obtained through direct fits to the SDSS total photometry, i.e., the cmodel magnitudes.

We then match the MPA/JHU stellar mass catalogue to the NYU-VAGC brighto sample and identify valid, unambiguous MPA/JHU stellar mass estimates for all but 32,327 (6.3\%) of the MGS galaxies. Although it is unclear what causes the failure in estimating stellar mass for these systems, they are statistically indistinguishable from the matched population in redshift, luminosity, flux, and colour. Therefore, given the tight scaling relationship between the NYU-VAGC stellar mass $M_{*}^{N}$ and the MPA/JHU mass $M_{*}^{M}$, we can recover the individual photometric stellar mass estimates for these unmatched galaxies in a way that is statistically consistent with the matched ones. We first divide the bright 0 galaxies into red and blue populations based on their $g-r$ colours $(>$ or $<0.8$; $\mathrm{k}$-corrected to $z=0.1$ ), because the scaling between the two types of estimates is colour-dependent and is much tighter within each single-colour population than the entire sample (Bell \& de Jong 2001). Within each colour, we then compute the probability distribution of $M_{*}^{M}$ at each fixed $M_{*}^{N}$ using the successfully matched galaxies, and assign each unmatched galaxy with $M_{*}^{N}$ a random stellar mass $M_{*}^{M}$ drawn from that distribution.

Stellar mass estimation is subject to various theoretical uncertainties in predicting the $M_{*} / L$, due to the choice of the SPS model, dust extinction law, stellar evolution model, and most importantly, the form of the IMF (see Conroy 2013 for a general review on these topics and L12 for a detailed discussion of the various systematic uncertainties in stellar mass estimation). Assuming no trend in IMF with galaxy type, environment, or redshif ${ }^{2}$ the range of different IMFs causes uncertainty in the absolute normalisation of the $M_{*} / L$ of factors of two to several depending on the passband (Bell \& de Jong 2001), thus shifting the inferred SMF horizontally while maintaining the same shape. The second major source of uncertainty lies in the difficulty in correctly measuring the total fluxes of individual galaxies from aperture photometry. The SDSS pipeline returns a fraction of the total light even when the cmodel magnitudes are used (Abazajian et al.2009), due to a combination of over-subtraction of the sky background and poor model fits to the light profiles (Bernardi et al. 2013). This issue is particularly acute for massive galaxies, where the measured number density of galaxies above $3 \times 10^{11} h^{-2} M_{\odot}$ may be underes-

\footnotetext{
1 http://home.strw.leidenuniv.nl/ jarle/SDSS/

2 For evidence suggesting non-universal IMFs, however, see van Dokkum \& Conroy 2010), Conroy \& van Dokkum 2012), Cappellari et al. (2012), and Ferreras et al. (2013).
}

timated by as much as a factor of five. Unfortunately, there is no well-calibrated correction that can be applied to the MPA/JHU catalogue. Therefore, we use the MPA/JHU catalogue as-is and focus on the particular mapping between the MPA/JHU stellar masses to dark matter halos. For the purpose of this mapping, both types of systematic errors on the stellar mass are rather benign, as long as the ranking order of galaxies in their stellar mass estimates is largely unperturbed. Unless otherwise noted, we will refer to the MPA/JHU stellar mass estimate simply as the "stellar mass" and denote it with $M_{*}$ for the rest of the paper.

\subsection{Stellar Mass Sample Selection}

The flux-limited nature of surveys like the SDSS makes it very difficult to select volume-limited galaxy samples thresholded or binned in stellar mass, due to the large spread in $M_{*} / L$ at fixed $M_{*}$. However, the $M_{*} / L$ distribution of the red, quiescent galaxies is much narrower than that of the blue, star-forming galaxies (Gallazzi \& Bell 2009), therefore at any given redshift the observed red population has a sharp cutoff at low stellar mass, where the observed galaxies are dominated by the blue population near the flux limit. This phenomenon is best illustrated in Figure 1a, where we show the average $g-r$ colour at each $\left(M_{*}, z\right)$ location, colourcoded by the colourbar on top. The map is well separated into two regimes based on the mixing of galaxy colours by the narrow streak of the $\langle g-r\rangle=0.8$ population, which we hereafter refer to as the "mixture" limit, analogous and related to the "flux" limit on a luminosity vs. $z$ diagram.

In Figure 19, above the mixture limit the variation of the average $g-r$ colour with $M_{*}$ is largely uniform across all redshifts below 0.1 , with a gradual and smooth transition at $M_{*} \sim$ $10^{10} h^{-2} M_{\odot}$ as a result of the galaxy evolution physics (a.k.a, downsizing; Cowie et al. [1996). The sharp colour transition across the mixture limit, however, is purely a manifestation of the fluxlimited nature of the sample, combined with the fact that quiescent galaxies produce much less light than their star-forming counterparts at the same $M_{*}$ due to their higher $M_{*} / L$ ratio. The sharpness of the transition corresponds to the narrow width of the $M_{*} / L$ distribution of these quiescent galaxies. Therefore, the mixture limit provides a simple empirical guideline for selecting stellar mass samples of relatively high volume-completeness, circumventing the problem of theoretically determining the maximum $M_{*} / L$ ratio for galaxies at the flux limit of each redshift.

Since our goal is to infer the stellar-to-halo mass mapping for the average galaxy population with a fair mix of both the quiescent and the star-forming galaxies, which occupy dark matter halos in different ways, we will restrict our analysis to the galaxies above the "mixture" limit (see Section 4.1 for an additional factor for making this choice of restriction). The functional form we adopt to describe the mixture limit $M_{*}^{\text {mix }}(z)$ is

$$
\lg \left(\frac{M_{*}^{\text {mix }}}{h^{-2} M_{\odot}}\right)=5.4 \times(z-0.025)^{0.33}+8.0,
$$

i.e., the white curves shown in Figure 1p and Figure 1, slightly more conservative than the $\langle g-r\rangle=0.8$ streak in Figure 1 . We then define different stellar mass samples above this mixture limit for the clustering and lensing measurements in Figure $1 \mathrm{p}$ and 1 , which show the distributions of galaxy number counts and number densities, respectively, on the $M_{*}-z$ diagram. The thick black boxes in Figure 1p represent a typical sample selection scheme adopted by the traditional HOD analysis. In order to predict the 

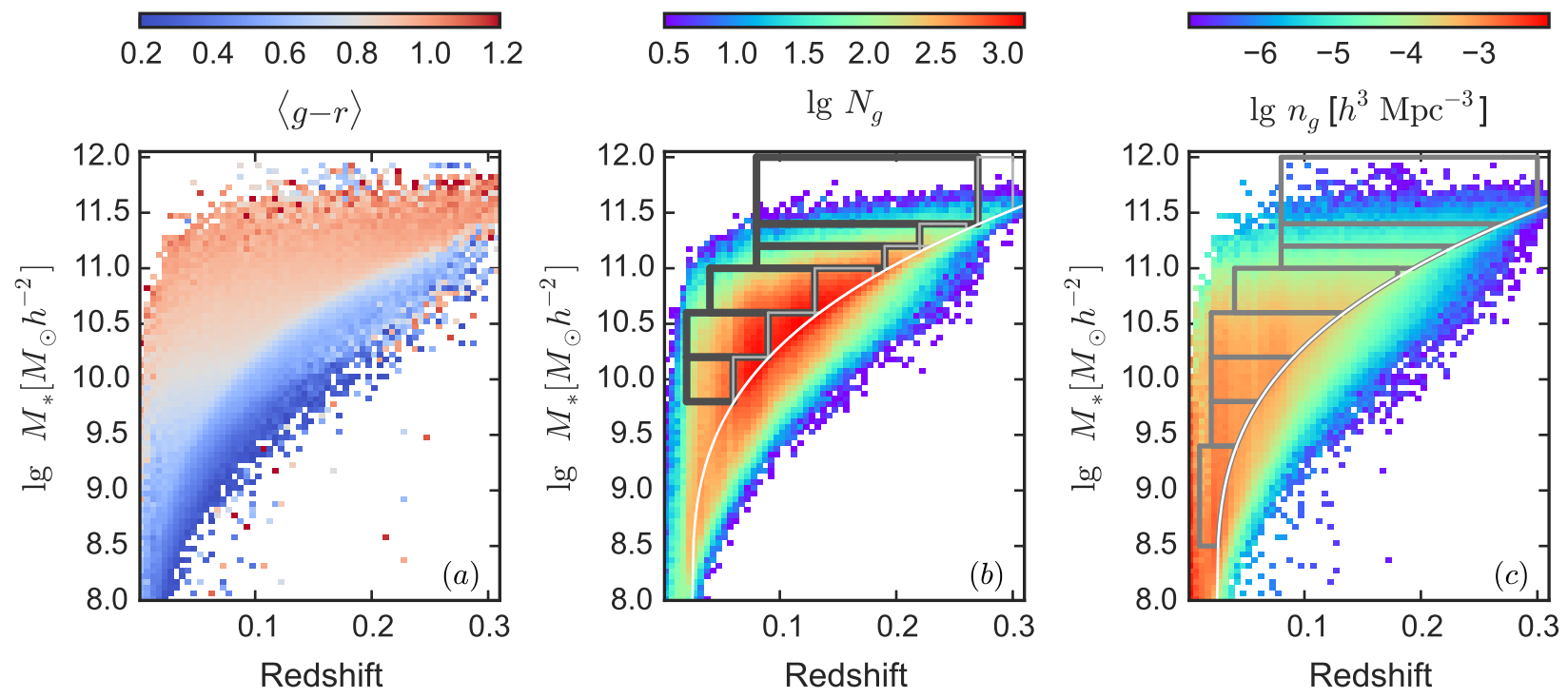

Figure 1. Distributions of the mean $g-r$ rest-frame colour (panel $a$ ), the observed number counts (panel $b$ ), and the co-moving number density (panel $c$ ) of SDSS DR7 galaxies on the stellar mass vs. redshift plane, with colourbars showing on the top. In the middle panel, the thick "boxes" indicate the galaxy samples selected for the traditional HOD analysis (CHOD), while the thin "wedges" represent the omitted region where galaxies are the most abundant. The gray regions in the right panel are the galaxy samples that were used for our fiducial i HOD analysis. The curved lower boundary of the selection is determined by the mixture limit below which the red galaxy population is severely underrepresented in the spectroscopic survey, and is derived from the distinctive white stripe $(\langle g-r\rangle=0.8)$ in the left panel (see Equation 1 .

Table 1. The two sets of stellar mass bins used for the iHOD and $\mathrm{CHOD}$ analyses, corresponding to the dark and gray thick selections in Figure $1 \mathrm{p}$ and 1 ; respectively. The $i \mathrm{HOD}$ analysis includes two extra stellar mass samples below $\lg M_{*}=9.8$, while the higher stellar mass samples have the same binning in $\lg M_{*}$ and the same minimum redshifts $z_{\text {min }}$ for the two analyses. However, all the i HOD samples have more extended redshift ranges, thresholded by the mixture limit defined in Equation [1] on the far side, so the maximum redshift $z_{\max }$ listed for each sample is the maximum redshift of any galaxies in that sample. For the $z_{\max }$ and $N_{g}$ we list the corresponding numbers for the CHOD analysis in parenthesis. The average large-scale bias for all, central, and satellite galaxies, the average logarithmic halo mass corresponding to the central galaxies, and the satellite fraction of each $i$ HOD sample (with $68 \%$ uncertainties) are derived from the fiducial $i$ HOD constraint for the corresponding i HOD samples.

\begin{tabular}{|c|c|c|c|c|c|c|c|c|}
\hline $\lg M_{*} / h^{-2} M_{\odot}$ & $z_{\min }$ & $z_{\max }$ & $N_{g}$ & $f_{\text {sat }}$ & $\left\langle b_{\text {all }}\right\rangle$ & $\left\langle b_{\text {sat }}\right\rangle$ & $\left\langle b_{\text {cen }}\right\rangle$ & $\left\langle\lg M_{h}^{\text {cen }} / h^{-1} M_{\odot}\right\rangle$ \\
\hline $8.5-9.4$ & 0.01 & 0.04 & 13,616 & $0.42_{-0.04}^{+0.04}$ & $1.12_{-0.02}^{+0.02}$ & $1.63_{-0.07}^{+0.07}$ & $0.74_{-0.02}^{+0.02}$ & $11.16_{-0.20}^{+0.16}$ \\
\hline $9.4-9.8$ & 0.02 & 0.06 & 16,247 & $0.42_{-0.04}^{+0.04}$ & $1.15_{-0.02}^{+0.01}$ & $1.66_{-0.06}^{+0.06}$ & $0.77_{-0.01}^{+0.01}$ & $11.44_{-0.09}^{+0.20}$ \\
\hline $9.8-10.2$ & 0.02 & $0.09(0.06)$ & $46,910(22,409)$ & $0.42_{-0.03}^{+0.04}$ & $1.19_{-0.01}^{+0.01}$ & $1.71_{-0.05}^{+0.05}$ & $0.82_{-0.01}^{+0.01}$ & $11.74_{-0.06}^{+0.05}$ \\
\hline $10.2-10.6$ & 0.02 & $0.13(0.09)$ & $96,946(58,209)$ & $0.37_{-0.02}^{+0.02}$ & $1.26_{-0.01}^{+0.01}$ & $1.83_{-0.04}^{+0.04}$ & $0.93_{-0.01}^{+0.01}$ & $12.15_{-0.04}^{+0.06}$ \\
\hline $10.6-11.0$ & 0.04 & $0.18(0.13)$ & $102,307(60,283)$ & $0.26_{-0.01}^{+0.01}$ & $1.40_{-0.01}^{+0.01}$ & $2.11_{-0.04}^{+0.04}$ & $1.15_{-0.01}^{+0.01}$ & $12.68_{-0.03}^{+0.02}$ \\
\hline $11.0-11.2$ & 0.08 & $0.22(0.19)$ & $24,908(19,506)$ & $0.17_{-0.01}^{+0.01}$ & $1.73_{-0.01}^{+0.01}$ & $2.65_{-0.06}^{+0.04}$ & $1.54_{-0.02}^{+0.01}$ & $13.21_{-0.02}^{+0.03}$ \\
\hline $11.2-11.4$ & 0.08 & $0.26(0.22)$ & $10,231(7,427)$ & $0.11_{-0.01}^{+0.02}$ & $2.13_{-0.02}^{+0.02}$ & $3.27_{-0.08}^{+0.06}$ & $1.99_{-0.03}^{+0.02}$ & $13.58_{-0.02}^{+0.02}$ \\
\hline $11.4-12.0$ & 0.08 & $0.30(0.27)$ & $3,137(2,649)$ & $0.05_{-0.01}^{+0.01}$ & $2.84_{-0.06}^{+0.06}$ & $4.28_{-0.13}^{+0.17}$ & $2.76_{-0.06}^{+0.06}$ & $13.96_{-0.03}^{+0.03}$ \\
\hline
\end{tabular}

clustering and lensing signals, the HOD model has to assume either volume-completeness of the sample within those boxes, or an ad hoc prescription describing the completeness as function of $M_{*}$ and/or $z$ (e.g., Miyatake et al. 2013). Both assumptions are less than ideal due to our ignorance about the $M_{*} / L$ ratio distribution of galaxies. Furthermore, the rectangular shape of the selections, imposed merely for the sake of modelling convenience, inevitably misses the regions where the galaxies are the most abundant (thin gray "wedges") on the 2D histogram because they occupy a much larger co-moving volume per unit redshift compared to the lower redshift "boxes". In principle the traditional HOD analysis can include more galaxies inside those "wedges" by adopting finer stellar mass bins with fewer galaxy per sample, but the measurement signal from each individual sample would be very noisy, rendering this scheme highly impractical. In this study we develop two novel improvements over the traditional HOD approach: 1) to statistically account for the sample incompleteness in a self-consistent way, and 2) to be able to predict the clustering and lensing signal for all the galaxies above the mixture limit (thick gray selections in Figure 1 ,). We hereafter refer to the traditional HOD approach as the $C H O D$ and our improved version the $i H O D$, where $c$ and $i$ are loosely tied to "completeness" and "incompleteness", respectively. Table 1 summarises the basic information of the two sets of sample selections used by the two modelling methods. In total, we select 314,302 ( $61 \%$ of the brighto sample) galaxies for the $i \mathrm{HOD}$ analysis, and from them 170,483 (54\% of the $i$ HOD galaxies) are used for the $C H O D$ analysis. 

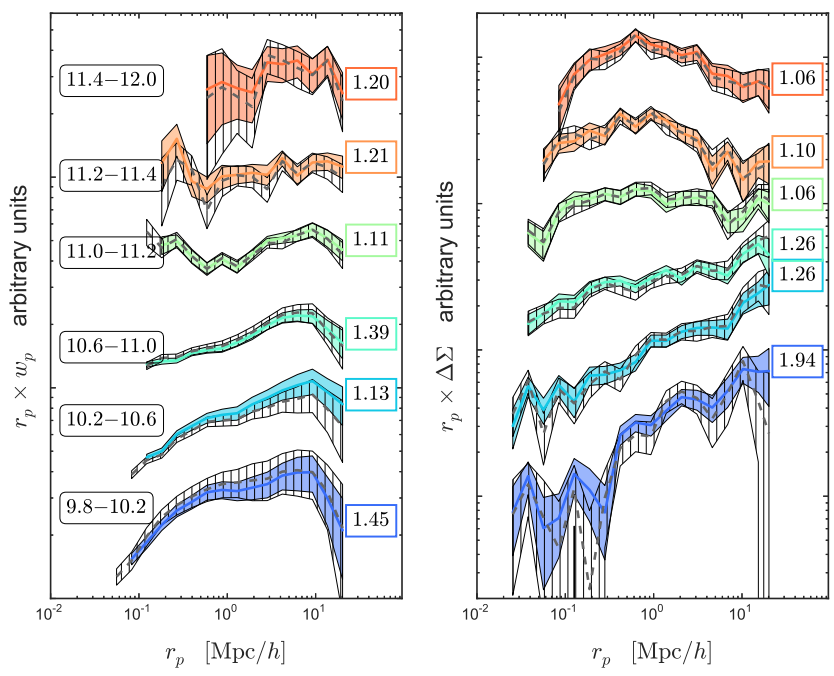

Figure 2. Comparison between the clustering (left) and lensing (right) measurements for the $i$ HOD (coloured solid) and CHOD (gray dashed) samples of the same stellar mass ranges, marked at the beginning of each pair of curves in the left panel. In each panel, the coloured and hatched bands represent the sizes of the uncertainties in the corresponding measurements, and the number marked at the end of each pair of bands is the ratio between the average signal-to-noise ratios of the $i$ HOD and the CHOD measurements, all $>1$ because of the extra galaxies included in the $i$ HOD samples. To avoid clutter, the units of the projected correlation function (left) and the g-g lensing signals (right) for each stellar mass bin are scaled arbitrarily.

\subsection{Source catalogue}

As sources for the g-g lensing measurement, we use a catalogue of background galaxies (Reyes et al. 2012) with a number density of $1.2 \operatorname{arcmin}^{-2}$ with weak lensing shears estimated using the re-Gaussianization method (Hirata \& Seljak 2003) and photometric redshifts from Zurich Extragalactic Bayesian Redshift Analyzer (ZEBRA, Feldmann et al.2006). The catalogue was characterised in several papers that describe the data, and use both the data and simulations to estimate systematic errors (see Reyes et al. 2012, Mandelbaum et al. 2012, Nakajima et al. 2012, Mandelbaum et al. 2013).

\section{MEASURING GALAXY CLUSTERING AND GALAXY-GALAXY LENSING}

Figure 2 compares the clustering and g-g lensing signals measured

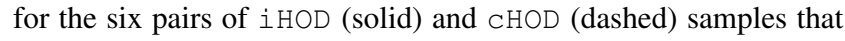
share the same stellar mass ranges (marked in the left panel). In each panel, the coloured and the hatched bands illustrate the sizes of the measurement uncertainties (i.e., the diagonal of the error matrices) in the 1 HOD and the CHOD cases, respectively. At the end of each pair of uncertainty bands, we mark the ratio between the average signal-to-noise ratios $(S / N)$ of the $i \mathrm{HOD}$ and the $\mathrm{CHOD}$ measurements. The improvement in the $S / N$ of the g-g lensing measurements (right panel) is consistent with the reduction in the Poisson errors by factors of $\sqrt{N_{g}^{i} / N_{g}^{c}}$, where $N_{g}^{i}$ and $N_{g}^{c}$ are the numbers of galaxies in the $i \mathrm{HOD}$ and the $\mathrm{CHOD}$ samples, respectively (the 4th column of Table 11. For the clustering measurements (left panel), the improvement for the three higher mass bins is consistent with the expected decrease in the Poisson error by $N_{g}^{i} / N_{g}^{c}$, while for the three lower mass bins the improvement is smaller than expected from assuming the errors are pure Poisson, due to the strong cosmic variance in these samples. These improvements in the $S / N$ of the measurements are crucial to the success of the $i$ HOD model in robustly solving the mapping between galaxies and halos without resorting to any external information (e.g., the SMF) or strong priors on the model parameters (e.g., the scatter in the SHMR).

We now describe how we measure the projected correlation function from galaxy pair counting and the surface density contrast from g-g lensing, and the reader who are familiar with the technical details can skip the remainder of this section.

\subsection{Projected Galaxy Correlation Function}

We measure the projected correlation function $w_{p}$ for each galaxy sample by integrating the $2 \mathrm{D}$ redshift-space correlation function $\xi^{s}$

$$
w_{p}\left(r_{p}\right)=\int_{-r_{\pi}^{\max }}^{+r_{\pi}^{\max }} \xi^{s}\left(r_{p}, r_{\pi}\right) d r_{\pi},
$$

where $r_{p}$ and $r_{\pi}$ are the projected and the line-of-sight (LOS) comoving distances between two galaxies. We measure the $w_{p}$ signal out to a maximum projected distance of $r_{p}^{\max }=20 h^{-1} \mathrm{Mpc}$, where the galaxy bias is approximately linear. For the integration limit, we adopt a maximum LOS distance of $r_{\pi}^{\max }=60 h^{-1} \mathrm{Mpc}$, where the average pairwise peculiar velocity is very small (Tinker et al. 2008). Based on a mildly modified linear Kaiser formalism, van den Bosch et al. (2013) showed that the $w_{p}$ measured with $r_{\pi}^{\max }=60 h^{-1} \mathrm{Mpc}$ should be boosted by a few to $8 \%$ depending on $r_{p}$, in order to correct for the residual redshift-space distortion (RRSD) effect due to the small but non-zero peculiar velocity beyond $r_{\pi}>60 h^{-1} \mathrm{Mpc}$ (see their figure 6). The boost was calibrated for a mock galaxy sample binned in luminosity with different cosmology, therefore it is not suitable to directly apply it to our measurement. However, the impact of the RRSD effect on our result, especially on the SHMR, can be roughly estimated as follows: in the most extreme case that all the constraining power comes from the clustering at large scales, based on the average bias vs. halo mass relation for the central galaxies listed in Table 1, we can infer that a $5 \%$ boost in $w_{p}$ translates to $2.5 \%$ in galaxy bias, which translates to to $\sim 0.015$ dex in halo mass, shifting the SHMR toward the lower mass end by $0.015 \mathrm{dex}$. This shift is much smaller than the scatter in the halo mass at fixed stellar mass and our constraint is not solely coming from $w_{p}$, therefore the impact of ignoring RRSD should be negligible.

We compute the 2D correlation using the Landy-Szalay estimator (Landy \& Szalay 1993),

$$
\xi^{s}\left(r_{p}, r_{\pi}\right)=\frac{D D-2 D R+R R}{R R},
$$

where $D D, D R$, and $R R$ represent the number counts of pairs of two data galaxies, one data and one random galaxies, and two random galaxies, respectively. The Landy-Szalay estimator has minimal variance and is insensitive to the number of random points (Kerscher et al. 2000). For each given galaxy, we find all the neighbouring galaxies within a cylinder of radius $r_{p}^{\max }$ and height $2 r_{\pi}^{\max }$ centered on that galaxy, using the libkd package within the Astrometry . net software (Lang et al. 2010). We then count all the pairs in each cell centered on $\left(r_{p}, r_{\pi}\right)$ and the three sets of pair counts thus directly give the value of $\xi^{s}\left(r_{p}, r_{\pi}\right)$ via Equation (3). 
The error covariance matrix for each $w_{p}$ measurement is estimated via the jackknife resampling technique (Norberg et al. 2009). We divide the entire footprint into 200 spatially contiguous, roughly equal-size patches on the sky and compute the $w_{p}$ for each of the 200 jackknife subsamples by leaving out one patch at a time. For each stellar mass sample, we adopt the sample mean of the 200 subsample measurements as our final estimate of $w_{p}$, and the sample covariance matrix as an approximate to the underlying error covariance.

The random galaxy catalogue was constructed following two steps. We first generate a sample of random positions on the sky with 10 times the size of the data catalogue, using the mangle software (Hamilton \& Tegmark|2004, Swanson et al.|2008) and the bright 0 angular selection function provided on the NYU-VAGC website. Secondly, we calculate the 2D joint distribution of stellar mass and redshift from the data catalogue, and draw 5131, 500 random pairs of $\left(M_{*}, z\right)$ values to assign to the stored random positions. In this way, we ensure the random galaxy catalogue has exactly the same angular, radial, and stellar mass joint selection functions as the data galaxies. We apply the same sample selection criterion to the random catalogue and the data sample. As mentioned in Section 2.1. we only use the $w_{p}$ values down to the physical distance that corresponds to the fibre radius at the maximum redshift of that sample.

The $w_{p}$ measurements for the eight iHOD stellar mass samples are shown as the solid circles with errorbars in the top subpanels in Figure 6 The errorbars reflect the diagonal components of the jackknife covariance matrices. Due to the strong cosmic variance effects in the low stellar mass samples $\left(\lg M_{*}<10.6\right)$, overall the off-diagonal components (not shown here) are strong and persist on both small and larges scales, while for the high stellar mass ones $\left(\lg M_{*}>11.0\right)$ they are only prominent on scales larger than $5 h^{-1} \mathrm{Mpc}$ and between two adjacent distance bins, i.e., along the diagonal blocks. We will refer back to Figure 6 and discuss in more detail the comparison between the measurements and the predictions from our best-fit model in Section 6

\subsection{Surface Density Contrast from Galaxy-Galaxy Lensing}

Here we describe how we measure the surface density contrast from g-g lensing. The lensing measurement begins with identification of background source galaxies around each lens (with photometric redshift larger than the lens spectroscopic redshift). Inverse variance weights are assigned to each lens-source pair, including both shape noise and measurement error terms in the variance:

$$
w_{l s}=\frac{1}{\Sigma_{\mathrm{crit}}^{2}\left(\sigma_{e}^{2}+\sigma_{\mathrm{SN}}^{2}\right)},
$$

where $\sigma_{e}^{2}$ is the shape measurement error due to pixel noise, and $\sigma_{\mathrm{SN}}^{2}$ is the RMS intrinsic ellipticity (both quantities are per component, rather than total; the latter is fixed to 0.365 following Reyes et al. 2012). $\Sigma_{\text {crit }}$ is the critical surface mass density defined by

$$
\Sigma_{\text {crit }}^{-1}\left(z_{l}, z_{s}\right) \equiv \frac{4 \pi G}{c^{2}} \frac{D_{l s} D_{l}\left(1+z_{l}\right)^{2}}{D_{s}},
$$

where $D_{l}$ and $D_{s}$ are the angular diameter distances to lens and source, and $D_{l s}$ is the distance between them. We use the estimated photometric redshift each source to compute $D_{s}$ and $D_{l s}$. The factor of $\left(1+z_{l}\right)^{2}$ comes from our use of co-moving coordinates.

The projected mass density in each radial bin can be computed via a summation over lens-source pairs " $l s$ " and random lens- source pairs " $r s$ ":

$$
\Delta \Sigma\left(r_{p}\right)=\frac{\sum_{l s} w_{l s} e_{t}^{(l s)} \Sigma_{\text {crit }}\left(z_{l}, z_{s}\right)}{2 \mathcal{R} \sum_{r s} w_{r s}},
$$

where $e_{t}$ is the tangential ellipticity component of the source galaxy with respect to the lens position, the factor of $2 \mathcal{R}$ converts our definition of ellipticity to the tangential shear $\gamma_{t}$, and $r_{p}$ is the comoving projected radius from the lens. The division by $\sum w_{r s}$ accounts for the fact that some of our 'sources' are physically associated with the lens, and therefore not lensed by it (see, e.g., Sheldon et al.2004). Finally, we subtract off a similar signal measured around random lenses, to subtract off any coherent systematic shear contributions (Mandelbaum et al. 2005); this signal is statistically consistent with zero for all scales used in this work.

To calculate the error bars, we also used the jackknife resampling method. As shown in Mandelbaum et al. (2005), internal estimators of error bars (in that case, bootstrap rather than jackknife) perform consistently with external estimators of errorbars for $\Delta \Sigma$ due to its being dominated by shape noise.

Use of photometric redshifts, which have nonzero bias and significant scatter, gives rise to a bias in the signals that can be easily corrected using the method from Nakajima et al. (2012). This bias is a function of lens redshift, and is properly calculated including all weight factors for each lens sample taking into account its redshift distribution. For typical lens samples in this work, the bias for which we apply a correction is of order 1 per cent, far below the statistical errors; the maximum is slightly below 10 per cent.

\section{MODEL FOR MAPPING THE STELLAR CONTENT TO HALOS}

\subsection{A Tale of Two HODs: cHOD vs. iHOD}

The key to statistically solving the mapping between stellar content and dark matter halos is $p\left(M_{*}, M_{h}\right)$, the $2 \mathrm{D}$ joint probability density distribution of a galaxy with stellar mass $M_{*}$ sitting in a halo of mass $M_{h}$, normalised so that $\iint p\left(M_{*}, M_{h}\right) \mathrm{d} M_{*} \mathrm{~d} M_{h}=1$. For analysing a galaxy sample with stellar mass range $\left[M_{*}^{0}, M_{*}^{1}\right]$ and redshift range $\left[z_{0}, z_{1}\right]$, it is common practice for HOD models to directly parameterize the occupation number as function of halo mass for the entire sample, $\left\langle N_{g}\left(M_{h}\right)\right\rangle$, which is related to $p\left(M_{*}, M_{h}\right)$ via

$$
\begin{aligned}
&\left\langle N_{g}\left(M_{h}\right)\right\rangle=\frac{n_{g}}{z_{1}-z_{0}}\left(\frac{\mathrm{d} n}{\mathrm{~d} M_{h}}\right)^{-1} \\
& \int_{z_{0}}^{z_{1}} \int_{M_{*}^{0}}^{M_{*}^{1}} p\left(M_{*}, M_{h}\right) f_{\text {obs }}\left(M_{*} \mid M_{h}, z\right) \mathrm{d} z \mathrm{~d} M_{*},
\end{aligned}
$$

where $\mathrm{d} n / \mathrm{d} M_{h}$ is the halo mass function, $n_{g}$ is the total galaxy number density in the Universe (both observed and unobserved), and $f_{\text {obs }}$ is the detection rate that varies between 0 and 1 . At any given redshift, the detection rate can be explicitly written as

$$
f_{\text {obs }}\left(M_{*} \mid M_{h}, z\right)=\int_{0}^{\Gamma_{\max } \equiv M_{*} / L_{\min }(z)} g\left(\Gamma \mid M_{*}, M_{h}\right) \mathrm{d} \Gamma,
$$

where $g\left(\Gamma \mid M_{*}, M_{h}\right)$ is the distribution of the stellar mass-to-light ratio $\Gamma \equiv M_{*} / L$ of galaxies at fixed $M_{*}$ within halos of mass $M_{h}$, and $L_{\min }(z)$ is the luminosity threshold corresponding to the flux limit at $z$. At low redshift, $L_{\min }(z)$ is low (large $\Gamma_{\max }$ ), so the integral extends over a wide range of $\Gamma$ values and gives a result that approaches 1 , while at higher redshift, $L_{\min }(z)$ is high (small 
$\Gamma_{\max }$ ), limiting the range of accessible $\Gamma$ values severely and thus lowering $f_{\text {obs }}$.

In order to describe the sample using a single HOD, $f_{\text {obs }}$ must be uniform across the redshift range, i.e., $f_{\text {obs }}\left(M_{*} \mid M_{h}, z\right) \equiv$ $f_{\mathrm{obs}}\left(M_{*} \mid M_{h}\right)$, which happens if and only if $f_{\mathrm{obs}}\left(M_{*} \mid M_{h}\right) \equiv 1$, so that

$$
\left\langle N_{g}\left(M_{h}\right)\right\rangle=n_{g}\left(\frac{\mathrm{d} n}{\mathrm{~d} M_{h}}\right)^{-1} \int_{M_{*}^{0}}^{M_{*}^{1}} p\left(M_{*}, M_{h}\right) \mathrm{d} M_{*} .
$$

Following the standard procedure in HOD modelling, we adopt Equation (9) for the CHOD model, writing separate contributions to $p\left(M_{*}, M_{h}\right)$ for central and satellite galaxies, and derive constraint by applying it to the samples defined in Figure 1 p. For the sake of comparison to the $i$ HOD constraint, we do not use the number density of sample galaxies as an input to the CHOD constraints.

However, due to the increase of the luminosity threshold with redshift for a flux-limited sample, the $M_{*}$-selected galaxy populations are strictly stratified in $z$ and the HODs at different redshifts must be treated separately. At fixed redshift $z$, the HOD is

$$
\begin{aligned}
& \left\langle N_{g}\left(M_{h} \mid z\right)\right\rangle= \\
& n_{g}\left(\frac{\mathrm{d} n}{\mathrm{~d} M_{h}}\right)^{-1} \int_{M_{*}^{0}}^{M_{*}^{1}} p\left(M_{*}, M_{h}\right) f_{\text {obs }}\left(M_{*} \mid M_{h}, z\right) \mathrm{d} M_{*} .
\end{aligned}
$$

Therefore, in order to predict $\left\langle N_{g}\left(M_{h} \mid z\right)\right\rangle$ from $p\left(M_{*}, M_{h}\right)$ we need to know $f_{\text {obs }}\left(M_{*} \mid M_{h}, z\right)$, which is inaccessible to us because of our ignorance of $g\left(\Gamma \mid M_{*}, M_{h}\right)$. However, we can rewrite the above Equation as

$$
\begin{aligned}
& \left\langle N_{g}\left(M_{h} \mid z\right)\right\rangle= \\
& \left(\frac{\mathrm{d} n}{\mathrm{~d} M_{h}}\right)^{-1} \int_{M_{*}^{0}}^{M_{*}^{1}} p\left(M_{h} \mid M_{*}\right)\left[\Phi\left(M_{*}\right) f_{\text {obs }}\left(M_{*} \mid M_{h}, z\right)\right] \mathrm{d} M_{*},
\end{aligned}
$$

by using Bayes' Theorem $p\left(M_{h} \mid M_{*}\right)=p\left(M_{*}, M_{h}\right) / p\left(M_{*}\right)$ and the definition of the parent $\operatorname{SMF} \Phi\left(M_{*}\right) \equiv n_{g} p\left(M_{*}\right)$. To make further progress in our predictions, we adopt the ansatz that above the mixture limit $M_{*}>M_{*}^{\mathrm{mix}}(z)$, the dependence of $f_{\mathrm{obs}}\left(M_{*} \mid M_{h}, z\right)$ on the halo mass is very weak, i.e., $f_{\text {obs }}\left(M_{*} \mid M_{h}, z\right) \sim f_{\text {obs }}\left(M_{*} \mid z\right)$. Applying this ansatz to Equation 11 we arrive at

$$
\left\langle N_{g}\left(M_{h} \mid z\right)\right\rangle=\left(\frac{\mathrm{d} n}{\mathrm{~d} M_{h}}\right)^{-1} \int_{M_{*}^{0}}^{M_{*}^{1}} p\left(M_{h} \mid M_{*}\right) \Phi_{\mathrm{obs}}\left(M_{*} \mid z\right) \mathrm{d} M_{*},
$$

where $\Phi_{\mathrm{obs}}\left(M_{*} \mid z\right)=\Phi\left(M_{*}\right) f_{\mathrm{obs}}\left(M_{*} \mid z\right)$ is the observed SMF at redshift $z$, directly accessible from the survey. For modelling the samples defined in Figure 1 1 for the $i \mathrm{HOD}$ analysis, we measure the observed galaxy SMF at each redshift, and then obtain the HOD for that redshift slice using Equation (12). In this way, we avoid the need to explicitly model the incompleteness as a function of $M_{*}$ and/or $M_{h}$. Although it appears from Equation (12) that both the amplitude and the shape of $\Phi_{\text {obs }}$ are used to derive $\left\langle N_{g}\left(M_{h} \mid z\right)\right\rangle$, in essence we only use the shape as an input to the iHOD constraint, because the normalisation of $\left\langle N_{g}\left(M_{h} \mid z\right)\right\rangle$ is irrelevant to the prediction of the clustering and lensing signals.

Before going any further, here we will lay out the theoretical arguments leading to the ansatz and defer the detailed discussion on its validation using consistency checks later in Section 6.2 and 7.1 Splitting the galaxies into red and blue populations explicitly in
Equation (8), we have

$$
\begin{array}{r}
f_{\text {obs }}\left(M_{*} \mid M_{h}, z\right)=\int_{0}^{\Gamma_{\max }(z)}\left\{f_{\text {red }}\left(M_{*} \mid M_{h}\right) g_{\text {red }}\left(\Gamma \mid M_{*}, M_{h}\right)\right. \\
\left.+\left[1-f_{\text {red }}\left(M_{*} \mid M_{h}\right)\right] g_{\text {blue }}\left(\Gamma \mid M_{*}, M_{h}\right)\right\} \mathrm{d} \Gamma, \quad(13)
\end{array}
$$

where $f_{\text {red }}\left(M_{*} \mid M_{h}\right)$ is the intrinsic fraction of red galaxies at given $M_{*}$ within halos of mass $M_{h}$. To understand the potential dependence of $f_{\text {obs }}\left(M_{*} \mid M_{h}, z\right)$ on the halo mass $M_{h}$, we first examine the variations of $f_{\text {red }}\left(M_{*} \mid M_{h}\right)$ and $g_{\text {red/blue }}\left(\Gamma \mid M_{*}, M_{h}\right)$ with $M_{h}$ separately and then combine them using the above Equation. Since the $M_{*} / L$ ratio $\Gamma$ is very tightly correlated with galaxy colour $c$ (Bell \& de Jong 2001), we can instead look at the colour distributions of the two populations, $g_{\text {red/blue }}\left(c \mid M_{*}, M_{h}\right)$, each with a centroid position $\bar{c}$ and a spread $\Delta c$. By analysing the variation in the galaxy colour bimodality with stellar mass and projected neighbour density using SDSS, Baldry et al. (2006) found that $\bar{c}$ and $\Delta c$ of the red and blue populations are stable across different environments of void, field, and groups and clusters (i.e., halo masses), while the red fraction $f_{\text {red }}$ increases continuously with $M_{*}$ and the local density of the environment, i.e., the so-called "mass" and "environmental" quenching, respectively (Peng et al. 2012). This stability in the colour distribution within each coloured population against the environment implies $g_{\text {blue }}\left(\Gamma \mid M_{*}, M_{h}\right) \equiv g_{\text {blue }}\left(\Gamma \mid M_{*}\right)$ and $g_{\text {red }}\left(\Gamma \mid M_{*}, M_{h}\right) \equiv g_{\text {red }}\left(\Gamma \mid M_{*}\right)$, so that Equation (13) can be simplified as

$$
\begin{aligned}
f_{\text {obs }}\left(M_{*} \mid M_{h}, z\right)= & f_{\text {red }}\left(M_{*} \mid M_{h}\right) G_{\text {red }}\left(M_{*} \mid z\right) \\
& +\left[1-f_{\text {red }}\left(M_{*} \mid M_{h}\right)\right] G_{\text {blue }}\left(M_{*} \mid z\right),
\end{aligned}
$$

where

$$
\begin{aligned}
G_{\text {red }}\left(M_{*} \mid z\right) & =\int_{0}^{\Gamma_{\max }(z)} g_{\text {red }}\left(\Gamma \mid M_{*}\right) \mathrm{d} \Gamma \\
G_{\text {blue }}\left(M_{*} \mid z\right) & =\int_{0}^{\Gamma_{\max }(z)} g_{\text {blue }}\left(\Gamma \mid M_{*}\right) \mathrm{d} \Gamma .
\end{aligned}
$$

are the fractions (from 0 to 1 ) of the red/blue galaxies with $M_{*}$ at $z$ that will be observed given the flux limit and the separate red/blue distributions of $M_{*} / L$ ratios. Above the mixture limit, the completeness of both the red and blue galaxies are relatively high, so that $G_{\text {red }}\left(M_{*} \mid z\right) \approx G_{\text {blue }}\left(M_{*} \mid z\right)=f_{\text {obs }}\left(M_{*} \mid z\right)$ and Equation (14) gives $f_{\text {obs }}\left(M_{*} \mid M_{h}, z\right)=f_{\text {obs }}\left(M_{*} \mid z\right)$. Below the mixture limit, however, the observed red galaxies are so scarce that $G_{\text {red }}\left(M_{*} \mid z\right) \sim 0$, yielding a halo mass-dependent $f_{\text {obs }}\left(M_{*} \mid M_{h}, z\right) \simeq\left[1-f_{\text {red }}\left(M_{*} \mid M_{h}\right)\right] G_{\text {blue }}\left(M_{*} \mid z\right) \quad$ because $f_{\text {red }}\left(M_{*} \mid M_{h}\right)$ is sensitive to $M_{h}$ (George et al. 2011).

We expect the ansatz to be largely valid except for the low mass samples $\left(M_{*}<10^{10} h^{-2} M_{\odot}\right)$ where the colour bimodality might shift with halo mass because of galaxy evolution (Taylor et al. 2015). We nonetheless proceed to apply this ansatz to the entire stellar mass range, relying on the fact that the statistical power of the low mass samples is quite low, and discuss the possible impact on our results later in Section 7.1 Finally, we emphasise that this ansatz assuming $f_{\text {obs }}\left(M_{*} \mid M_{h}, z\right) \sim f_{\text {obs }}\left(M_{*} \mid z\right)$, explicitly made in the $i$ HOD model to account for the fact that for any given $M_{*}$ we observed fewer intrinsically high- $M_{*} / L$ systems at higher redshifts, is a much weaker assumption than required by the traditional HOD models, which assume all the high $-M_{*} / L$ systems were detected in the sample, i.e., $f_{\text {obs }}\left(M_{*} \mid M_{h}, z\right)=1$. In other words, since $f_{\text {obs }}\left(M_{*} \mid M_{h}, z\right)=1$ is a sufficient condition for $f_{\mathrm{obs}}\left(M_{*} \mid M_{h}, z\right)=f_{\mathrm{obs}}\left(M_{*} \mid z\right)$, the i HOD model includes the CHOD model as a subset. 
It is important to point out that during the constraint, the $i \mathrm{HOD}$ model remains entirely agnostic of the overall amplitude of the SMF, whether it be the parent SMF (normalised by $n_{g}$ ) or the observed ones (normalised by the product of $n_{g}$ and $f_{\text {obs }}\left(M_{*} \mid z\right)$ ), because neither $n_{g}$ nor $f_{\text {obs }}\left(M_{*} \mid z\right)$ is known a priori. However, the $i$ HOD model is built on top of the halo mass function, which has a fixed normalisation in any given $\Lambda \mathrm{CDM}$ cosmology. Thus, once constrained by the clustering and lensing data, the best-fit iHOD model would give an explicit prediction for both the shape and the amplitude of the parent SMF. This prediction automatically gives an estimate of $f_{\text {obs }}\left(M_{*} \mid z\right)$ when compared to the observed SMF at redshift $z$. The estimated $f_{\text {obs }}\left(M_{*} \mid z\right)$ thus provides a useful consistency check of the iHOD model. Any significant departure of $f_{\text {obs }}$ from unity at stellar mass above the mixture limit would indicate failure of our model assumptions (e.g., the ansatz about the weak dependence of $f_{\text {obs }}\left(M_{*} \mid z\right)$ on $\left.M_{h}\right)$ and/or degeneracies in the model parameters (e.g., residual degeneracy between scatter and amplitude of the SHMR), while a successful model, as we will discover in Section 7.1 would reproduce $f_{\text {obs }}$ curves that are slightly below unity. Another consistency check is the comparison between the SHMR constraints from the CHOD and the $i \mathrm{HOD}$ models on the high mass end - in the regime of $M_{*} \gg M_{*}^{\operatorname{mix}}(z)$ where $f_{\text {obs }}\left(M_{*} \mid M_{h}, z\right) \rightarrow 1$, the iHOD model becomes identical to the $\mathrm{CHOD}$ model and the two set of constraints, as will be shown later in Section 6.2 should be consistent with each other.

In summary, both the $C H O D$ and $i$ HOD models rely on the prediction of $p\left(M_{*}, M_{h}\right)$. The primary difference is that the $\mathrm{CHOD}$ model employs a single HOD by assuming that the galaxy sample is volume-complete in stellar mass, while the iHOD model is able to self-consistently take into account the redshift-dependent selection function of the samples by working in narrow redshift slices and using information from the shape of the observed galaxy SMF at each slice. This improvement in $\mathrm{i}$ HOD enables us to include $84 \%$ more galaxies than used in $\mathrm{CHOD}$ by adding the low mass galaxies $\left(M_{*}<10^{9.8} h^{-2} M_{\odot}\right)$ in the local universe as well as more distant galaxies, hence the significant improvement in the $S / N$ of the measurements shown in Figure 2 This process cannot be extended indefinitely, since at lower stellar mass (below the mixture limit for a given redshift) the basic assumption behind $i$ HOD fails, but the range of stellar masses where it is applicable is still large enough for significant improvements.

\subsection{Deriving the Two HODs}

Here we describe the theoretical framework for predicting the HODs for both the CHOD and 1 HOD models. In particular, we first model the total number of galaxies (both observed and unobserved) per log-stellar mass within halos of some fixed mass, $\mathrm{d} N\left(M_{*} \mid M_{h}\right) / \mathrm{d} \lg M_{*}$, and then compute the joint probability as

$$
p\left(M_{*}, M_{h}\right)=\frac{\lg e}{M_{*} n_{g}} \frac{\mathrm{d} N\left(M_{*} \mid M_{h}\right)}{\mathrm{d} \lg M_{*}} \frac{\mathrm{d} n}{\mathrm{~d} M_{h}} .
$$

To conform to the traditional HOD notation, we hereafter refer to $\mathrm{d} N\left(M_{*} \mid M_{h}\right) / \mathrm{d} \lg M_{*}$ simply as $\left\langle N\left(M_{*} \mid M_{h}\right)\right\rangle$ by implicitly assuming $\Delta \lg M_{*} \equiv 0.02$ throughout the paper. Given $p\left(M_{*}, M_{h}\right)$, we can use Equation 9 to specify a single HOD for each of the CHOD samples. For the $i$ HOD analysis, however, we also need to compute the parent stellar mass function

$$
\Phi\left(M_{*}\right)=n_{g} \int_{0}^{+\infty} p\left(M_{*}, M_{h}\right) \mathrm{d} M_{h},
$$

and extract the observed stellar mass function $\Phi_{\text {obs }}\left(M_{*}\right)$ directly from the data. The distribution of host halo mass for galaxies at fixed stellar mass is simply

$$
p\left(M_{h} \mid M_{*}\right)=\frac{p\left(M_{*}, M_{h}\right)}{p\left(M_{*}\right)}=\frac{n_{g} p\left(M_{*}, M_{h}\right)}{\Phi\left(M_{*}\right)},
$$

and we can obtain the HODs for individual redshift slices within each $i$ HOD sample using Equation (12). Since the redshift range (0.02-0.30) spans $\sim 3$ billion years during which only $8 \%$ of the total stellar mass observed today formed (cf. equation 15 in Madau \& Dickinson 2014), we assume $p\left(M_{*} \mid M_{h}\right)$ to be constant with redshift. Therefore, all the redshift evolution in the theoretical model comes from the cosmic growth in the halo mass function. To speed up the calculation without loss of accuracy, we adopt the same halo mass function for all the redshift slices within each sample, calculated at the volume-averaged redshift of that sample. We have tested this approximation by comparing the predicted signals with those computed from integrating the halo mass functions over all the redshift slices and the difference is negligible.

\subsection{Parameterizing $\left\langle N\left(M_{*} \mid M_{h}\right)\right\rangle$}

Our analytic model for $\left\langle N\left(M_{*} \mid M_{h}\right)\right\rangle$ has two components: 1) the mean and the scatter of the SHMR for the central galaxies, the combination of which automatically specifies $\left\langle N_{\text {cen }}\left(M_{*} \mid M_{h}\right)\right\rangle$, and 2) the mean number of satellite galaxies with stellar mass $M_{*}$ inside halos of mass $M_{h},\left\langle N_{\text {sat }}\left(M_{*} \mid M_{h}\right)\right\rangle$. We adopt a similar parameterization for the two components as in L11, but allow more of them to vary during the constraint, and predict the signals differently. Here we will briefly describe the functional forms and the model parameterizations.

At fixed halo mass, we assume a log-normal probability distribution for the stellar mass of the central galaxies, with a log-normal scatter. The mean SHMR is then the sliding mean of the log-normal distribution as a function of the halo mass, $f_{\text {SHMR }} \equiv \exp \left\langle\ln M_{*}\left(M_{h}\right)\right\rangle$. The L11 functional form for $f_{\text {SHMR }}$ that we adopt for our analysis is defined by Behroozi et al. (2010) via its inverse function,

$$
M_{h}=M_{1} m^{\beta} \exp \left(\frac{m^{\delta}}{1+m^{-\gamma}}-\frac{1}{2}\right),
$$

where $m \equiv M_{*} / M_{*, 0}$. Among the five parameters that describe $f_{\mathrm{SHMR}}, M_{1}$ and $M_{*, 0}$ are the characteristic halo mass and stellar mass that separate the behaviours in the low and high mass ends $\left(f_{\operatorname{SHMR}}\left(M_{1}\right)=\ln M_{*, 0}\right)$. The inverse function starts with a low-mass end slope $\beta$, crosses a transitional regime around $\left(M_{*, 0}\right.$, $M_{1}$ ) dictated by $\gamma$, and reaches a high-mass end slope $\beta+\delta$. The Figure 1 in L11 illustrates the different responses of $f_{\text {SHMR }}$ to the changes in each of the five parameters.

The log-normal scatter at fixed halo mass is the quadratic sum of the intrinsic scatter and the measurement error. It is important to keep in mind that the intrinsic part of this scatter must be the same for all studies, while studies with different datasets or stellar mass determination methods may have differing measurement error contributions and thus a different total log-normal scatter. L12 considered two models for the scatter, one that is constant and another that includes an empirical stellar mass-dependence in the measurement error across the entire mass range. They found that since the constraint on the overall scatter is primarily driven by the high mass end where the slope of $f_{\mathrm{SHMR}}$ is much shallower, the mass-dependence of the scatter at the low mass end has little impact on their results. In light of this finding and to focus on the 


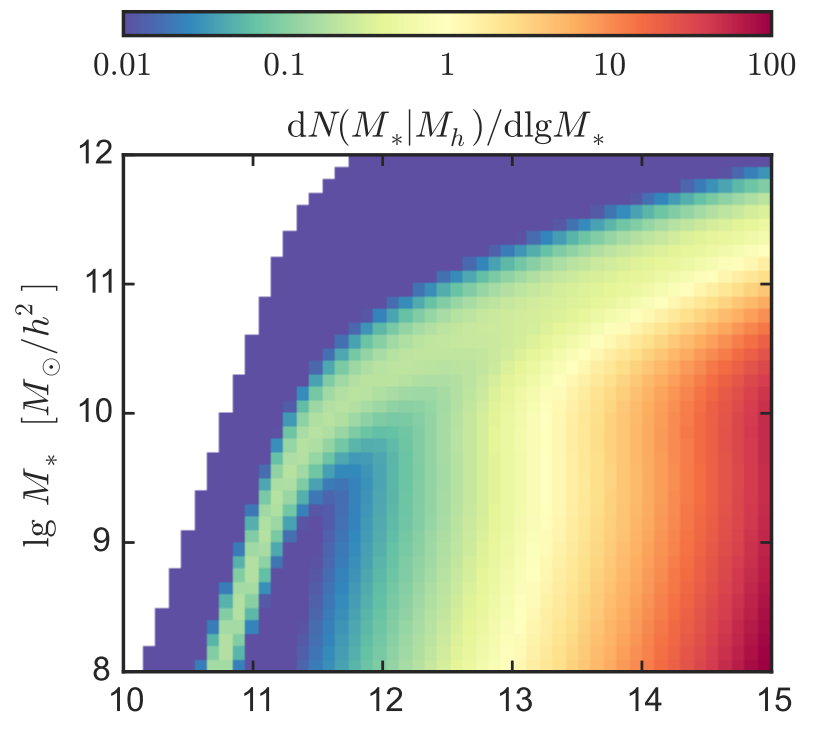

$\lg M_{h} \quad\left[M_{\odot} / h\right]$

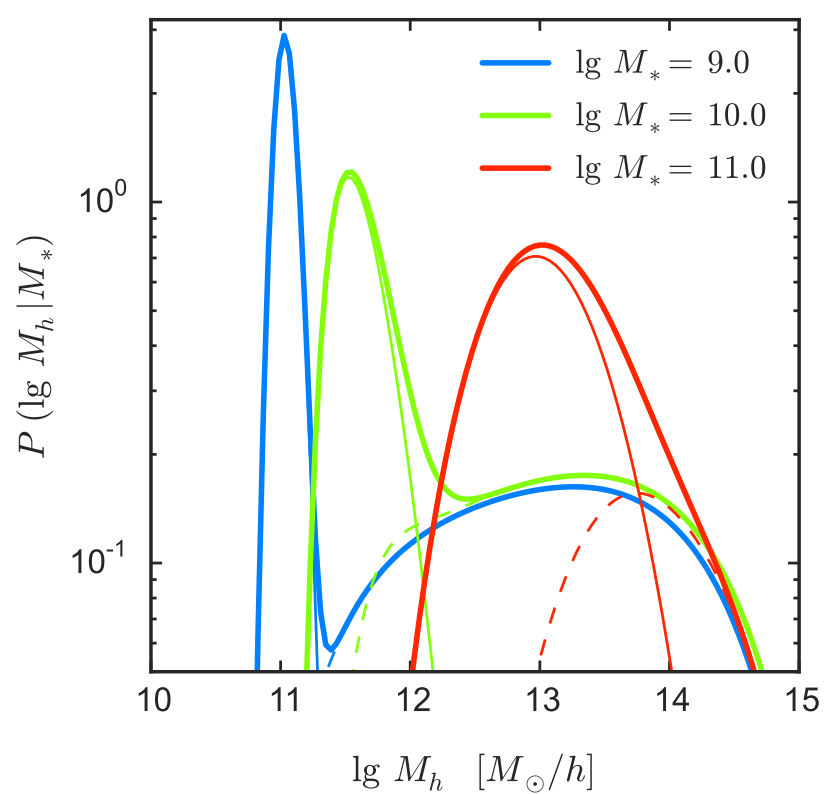

$\lg M_{h} \quad\left[M_{\odot} / h\right]$

Figure 3. Left Panel: The 2D HOD, i.e., the average number of galaxies per dex in stellar mass within halos at fixed mass, predicted by the best-fit i HOD model. Right Panel: The probability distributions of the host halo mass for galaxies at three fixed log-stellar mass listed on the top right. Thick solid, thin solid, and thin dashed lines represent contributions from all, central, and satellite galaxies, respectively. Galaxies of higher stellar mass are more likely to be central galaxies sitting in high mass halos, whereas low mass galaxies are as likely to be satellites of high mass halos as centrals of their own halos.

scatter at the high mass end, we keep the scatter independent of halo mass below $M_{1}$, but allow more freedom in the scatter above the characteristic mass scale, with an extra component that is linear in $\lg M_{h}$ :

$$
\sigma_{\ln M_{*}}\left(M_{h}\right)= \begin{cases}\sigma_{\ln M_{*}}, & M_{h}<M_{1} \\ \sigma_{\ln M_{*}}+\eta \lg \frac{M_{h}}{M_{1}}, & M_{h} \geqslant M_{1}\end{cases}
$$

The motivation behind this additional degree of freedom is two-fold: 1) the average measurement uncertainty of the stellar mass estimates decrease with $M_{*}$ (hence $M_{h}$ ) in the MPA/JHU catalogue, and 2) recently, there is evidence suggesting a smaller scatter at the high mass end of the SHMR (e.g., Shankar et al. 2014), although in Equation (20) the scatter is allowed to either increase or decrease with halo mass. The combination of the mean and the scatter fully specifies the HOD of central galaxies,

$$
\begin{aligned}
& \left\langle N_{\text {cen }}\left(M_{*} \mid M_{h}\right)\right\rangle= \\
& \frac{1}{\sigma_{\ln M_{*}}\left(M_{h}\right) \sqrt{2 \pi}} \exp \left[-\frac{\left[\ln M_{*}-\ln f_{\text {SHMR }}\left(M_{h}\right)\right]^{2}}{2 \sigma_{\ln M_{*}}^{2}\left(M_{h}\right)}\right] .
\end{aligned}
$$

Follow L11, we model $\left\langle N_{\text {sat }}\left(M_{*} \mid M_{h}\right)\right\rangle$ as the derivative of the satellite occupation number in stellar mass-thresholded samples, $\left\langle N_{\text {sat }}\left(>M_{*} \mid M_{h}\right)\right\rangle$, which is parameterized as a power of halo mass with an exponential cutoff and scaled to $\left\langle N_{\text {cen }}\left(>M_{*} \mid M_{h}\right)\right\rangle$ as follows,

$$
\begin{aligned}
& \left\langle N_{\text {sat }}\left(>M_{*} \mid M_{h}\right)\right\rangle= \\
& \quad\left\langle N_{\text {cen }}\left(>M_{*} \mid M_{h}\right)\right\rangle\left(\frac{M_{h}}{M_{\text {sat }}}\right)^{\alpha_{\text {sat }}} \exp \left(\frac{-M_{\text {cut }}}{M_{h}}\right) .
\end{aligned}
$$

Instead of fixing $\alpha_{\text {sat }}$ to be 1 as in L11, we allow it to vary during the fit. We parameterize both the characteristic mass of a single satellite-hosting halo, $M_{\text {sat }}$, and the cutoff mass scale, $M_{\text {cut }}$, as simple power law functions of the threshold stellar mass, so that

$$
\frac{M_{\mathrm{sat}}}{10^{12} h^{-2} M_{\odot}}=B_{\mathrm{sat}}\left(\frac{f_{\mathrm{SHMR}}^{-1}\left(M_{*}\right)}{10^{12} h^{-2} M_{\odot}}\right)^{\beta_{\mathrm{sat}}},
$$

and

$$
\frac{M_{\text {cut }}}{10^{12} h^{-2} M_{\odot}}=B_{\text {cut }}\left(\frac{f_{\text {SHMR }}^{-1}\left(M_{*}\right)}{10^{12} h^{-2} M_{\odot}}\right)^{\beta_{\text {cut }}},
$$

respectively. In practice, we choose a 0.02 dex bin size in stellar mass for the numerical differentiation of $\left\langle N_{\text {sat }}\left(>M_{*} \mid M_{h}\right)\right\rangle$.

The left panel of Figure 3 displays the $\left\langle N\left(M_{*} \mid M_{h}\right)\right\rangle$ map predicted by the best-fit $i$ HOD model in our analysis. The SHMR of the central galaxies is clearly seen as the "main sequence" enveloping the "cloud" occupied by the satellite galaxies. The right panel shows the probability distribution of host halo masses for galaxies at $M_{*}=10^{10} h^{-2} M_{\odot}$ (blue), $10^{11} h^{-2} M_{\odot}$ (green), and $10^{12} h^{-2} M_{\odot}$ (red), each computed from the map in the left panel using the combination of Equations [16, , 17), and (18). The thin solid and the dashed curves of each colour show the contributions to the total probability distribution from the central and the satellite galaxies, respectively. The central galaxy contribution shows an increasing logarithmic scatter in halo mass with stellar mass, mainly due to the change of slope in the SHMR when going to the high mass end (where $\sigma_{\ln M_{*}}$ shrinks slightly as well, i.e., $\eta<0$ ). The lower mass satellites, however, populate halos of much greater diversity than their high mass counterparts. Meanwhile, the satellite fraction increases toward lower stellar masses, but the total number of galaxies is dominated by central galaxies at all stellar masses.

\subsection{Spatial Distribution of Galaxies within Halos}

In addition to the parameterization of $\left\langle N\left(M_{*} \mid M_{h}\right)\right\rangle$, we also need to model the spatial distribution of galaxies within dark matter halos for the small-scale clustering and lensing predictions. We 
assume the isotropic Navarro-Frenk-White (NFW: Navarro et al. 1997) density profile for halos with a concentration-mass relation $c_{\mathrm{dm}}\left(M_{h}\right)$ calibrated from simulations (described further below). Although the NFW density profile is regarded as "universal" only in pure dark matter simulations where the effects of baryons are absen ${ }^{3}$ recent studies of nearby rich clusters found that, despite the fact that the baryons tend to flatten the dark matter distribution in the cluster centre, the total matter density (i.e., baryon and dark matter combined) still maintains a NFW shape from the scales of the central brightest cluster galaxy (BCG) out to the virial radius (Newman et al. 2013), probably due to the significant mixing between stars and dark matter as a result of frequent mergers (Laporte \& White 2014). For smaller systems like the group and galaxy-scale halos with $M_{h}<10^{14} h^{-1} M_{\odot}$, however, the sum of an NFW and a stellar mass component is required to explain the inner slope of the observed total matter density profiles (Mandelbaum et al. 2006. Newman et al. 2015). In light of these observational findings, we add a model-independent stellar mass component as a point source in the halo centre to the g-g lensing predictions for all the galaxy samples, so that

$$
\Delta \Sigma_{\text {stellar }}=\frac{\left\langle M_{*}\right\rangle}{\pi r_{p}^{2}}
$$

where $\left\langle M_{*}\right\rangle$ is the average stellar mass of each stellar mass sample. Although this extra stellar component is not necessary for the central galaxies of very rich clusters, i.e., some galaxies within the highest stellar mass sample, the minimum fitting scale for that sample is above $0.1 h^{-1} \mathrm{Mpc}$ (see Appendix A, where the stellar contribution to $\Delta \Sigma$ calculated via Equation 25] is negligible.

Central galaxies are placed at the centres of the NFW halos. For the modelling of $w_{p}$, we do not consider the miscentering effect, which is likely to be important only for around $30 \%$ of the BCGs (George et al. 2012). In our analysis the galaxy clustering for BCGs is measured down to $\sim 0.2 h^{-1} \mathrm{Mpc}$, therefore largely immune to the miscentering effect which has a kernel $\simeq 75 h^{-1} \mathrm{kpc}$ (George et al. 2012). It is worth noting that the miscentering issue in our case is more benign than in other recent papers that have explicitly modelled it (e.g., Miyatake et al. 2013, More et al. 2014). In those papers, the samples that were being modelled had strict colour and luminosity selection, such that the central galaxy in the halo might not be present in the sample. In our case, with a flux-limited sample that goes to a relatively low flux limit, the central galaxies in the vast majority of group- and clustersize halos should be present in the sample, so we only have to contend with small offsets of the central galaxy from the halo centre (rather than a complete misidentification of the central galaxy). For the g-g lensing, the miscentering effect is compounded and somewhat cancelled by the contribution from the subhalo of satellite galaxies (Yoo et al. 2006), which is more important for low stellar mass samples. We will discuss the modelling of the combined effects further in Section 5.3

We assume an NFW profile for the satellite distribution as well, but with a different amplitude of the concentration-mass relation than the dark matter. In particular, we set $c_{\text {sat }}\left(M_{h}\right) \equiv$ $f_{c} \times c_{\mathrm{dm}}\left(M_{h}\right)$, where $f_{c}$ characterises the spatial distribution of satellite galaxies relative to the dark matter within halos.

To summarise, we have in total 13 model parameters. Among them $\left\{\lg M_{h}^{1}, \lg M_{*}^{0}, \beta, \delta, \gamma\right\}$ describe the mean SHMR,

3 However, see Gao et al. 2008) and Dutton \& Macciò (2014) regarding potentially better "universality" when using the Einasto (1965) profile.
$\left\{B_{\text {sat }}, \beta_{\text {sat }}, B_{\text {cut }}, \beta_{\text {cut }}, \alpha_{\text {sat }}\right\}$ describe the parent HOD of satellite galaxies, $\left\{\sigma_{\ln M_{*}}, \eta\right\}$ describe the logarithmic scatter about the mean SHMR, and $f_{c}$ is the ratio between the concentrations of the satellite distribution and the dark matter profile.

\section{PREDICTING SIGNALS FROM THE HALO MODEL}

\subsection{Prerequisites and Approximations}

The goal of our analysis is to infer the SHMR of central galaxies and the HOD of satellite galaxies using the galaxy clustering and the g-g lensing. From the inferred $\left\langle N\left(M_{*} \mid M_{h}\right)\right\rangle$, we can predict the parent stellar mass function to compare to those empirically reconstructed from the $V_{\max }$ method. This route is exactly the reverse of the methodology employed in the SHAM studies (e.g., M10), which infer the SHMR by abundance matching to the $V_{\max }-$ estimated SMF and then predict the clustering and/or lensing as a cross-check.

To facilitate a direct comparison with the SHAM results, we adopt the same flat $\Lambda \mathrm{CDM}$ cosmology model as in M10 (listed in Section 11. For the linear matter power spectrum, we use the low-baryon transfer function of Eisenstein \& Hu (1998), which is a good approximation to the full transfer function on scales well below the BAO scale. To compute the non-linear matter correlation function $\xi_{m m}$, we use the prescription from Takahashi et al. (2012) (an updated version of the halofit prescription from Smith et al. 2003) to generate the non-linear power spectrum for Fourier transforming to $\xi_{m m}$. The halo mass function and the halo bias function are from Tinker et al. (2008) and Tinker et al. (2010), respectively. The halo mass-concentration relationship $c_{\mathrm{dm}}\left(M_{h}\right)$ is from the fitting formula of Zhao et al. (2009), which accurately recovers the flattening of halo concentration at high masses. (In DR7 we do not expect an upturn, which only shows up at redshifts beyond 1; see Prada et al. 2012).

For the $C H O D$ samples the signal prediction is relatively straightforward, as only one HOD is required to describe each sample. We describe the prediction of $w_{p}$ and $\Delta \Sigma$ from a single HOD in Section 5.2 Complexity arises when predicting the signals for the $i$ HOD samples, each of which contains multiple HODs that describe the stratification of galaxy populations due to the redshiftdependent selection function. We divide each i HOD sample into multiple redshift slices of $\Delta z=0.01$, which corresponds to a comoving width of $\sim 30 h^{-1} \mathrm{Mpc}$ across the redshift range of the MGS. This redshift bin size is chosen to make sure the slices are: 1) thin enough for the redshift selection function to be uniform, and 2) thick enough to include both the one and two-halo terms in the LOS direction internally. Within each redshift slice $i$ we predict the clustering and lensing signals, $w_{p}^{i}$ and $\Delta \Sigma^{i}$, in the same way as in the $C H O D$ analysis, and combine them to obtain the final predictions for the full sample,

$$
w_{p}=\left(\sum_{i} N_{i}^{2} w_{p}^{i}\right) /\left(\sum_{i} N_{i}^{2}\right),
$$

and

$$
\Delta \Sigma=\left(\sum_{i} N_{i} w_{i} \Delta \Sigma^{i}\right) /\left(\sum_{i} N_{i} w_{i}\right),
$$

where $N_{i}$ is the number of observed galaxies in slice $i$ and $w_{i}$ is the per-galaxy lensing weight at each redshift. $w_{i}$ is essentially related to the inverse variance weights $w_{l s}$ defined in Equation (4), but integrated over the source redshift distribution. It also includes the 

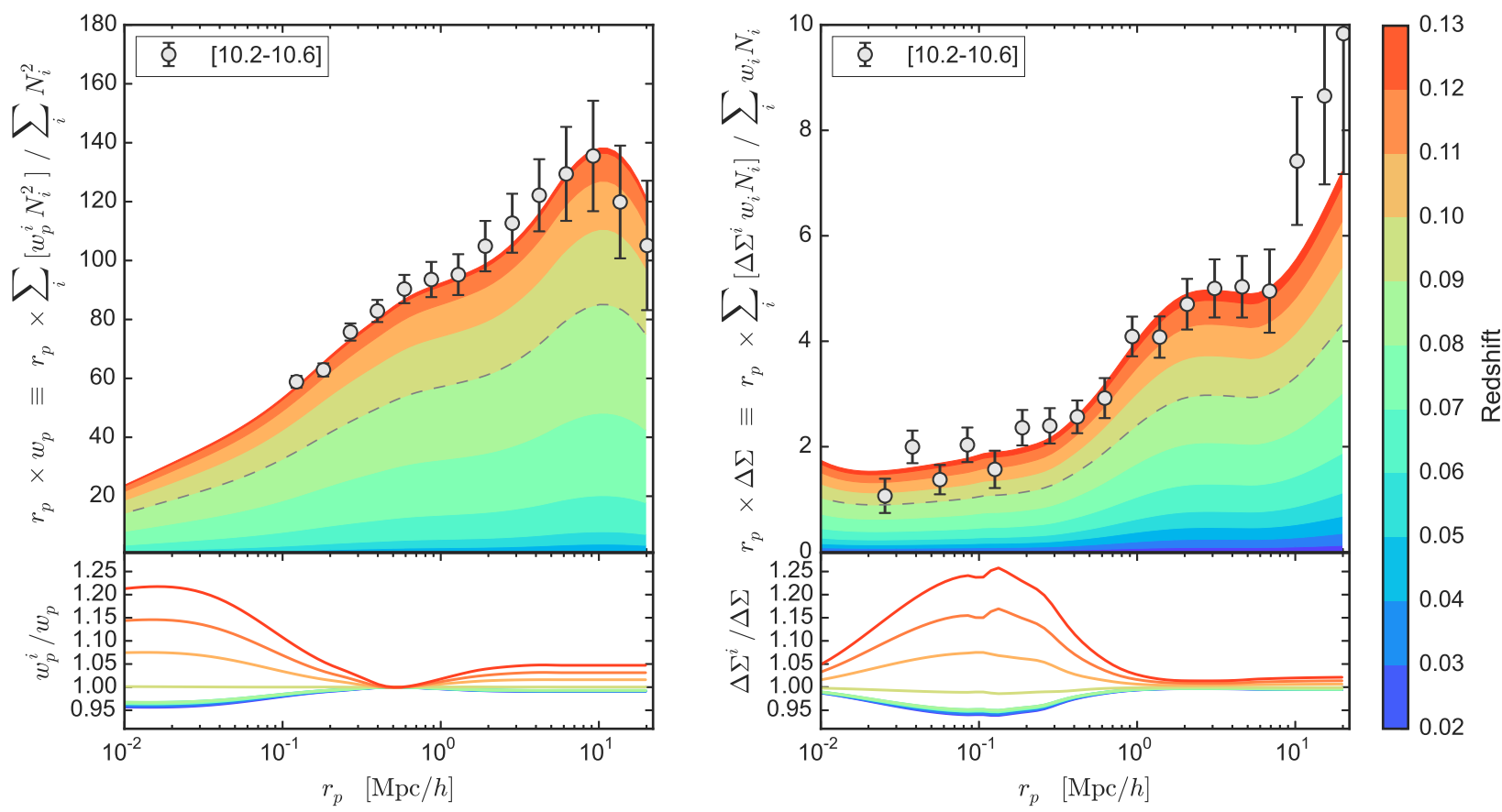

Figure 4. Contributions from individual redshift slices to the predicted clustering (left) and lensing (right) signals of the iHOD sample with $\lg M_{*}=[10.2,10.6]$. In each panel, the top sub-panel compares the measured signal (circles with errorbars) to the predicted total signal, decomposed into the contributions from each of the 11 redshift slices (colour-coded by the colourbar on the right). The dashed curve in each stack marks the maximum redshift (0.09) of the CHOD sample with the same stellar mass range, indicating a $\sim 40 \%$ gain in the total signal by switching from $\mathrm{CHOD}$ to $i$ HOD. Due to the difference in the weighting schemes, the fractional contributions of the same redshift slice to the total signals are different in the two cases. The bottom sub-panels show the ratio of the signal predicted for each slice over the signal for the whole sample. In both the clustering and lensing cases, the fractional variation is coherent with redshift and below $25 \%$ on all scales.

geometric factor $\left(1 / \Sigma_{c}^{2}\right)$ as well as the fact that an annular bin with a fixed centre value of $r_{p}$ and width $\Delta r_{p}$ contains more source galaxies at lower redshift, due to its larger area on the sky. Since the source catalogue is the same for the $\mathrm{g}$-g lensing in each redshift slice, the pair counting weight for $\Delta \Sigma^{i}$ is $\propto N_{i}$ instead of $\propto N_{i}^{2}$.

Figure 4 illustrates the fractional contributions of each individual redshift slice to the predicted total signal (calculated from the two equations above; top sub-panels), and compares the fractional variations among the predicted signals of all redshift slices (bottom sub-panels), using the $i$ HOD sample with $\lg M_{*}=[10.2,10.6]$ as an example. The dashed curve in each top sub-panel indicates the maximum redshift (0.09) of the corresponding $\mathrm{CHOD}$ sample with the same stellar mass range. Clearly, the three extra redshift slices above $z=0.09$ included by the iHOD analysis contribute $\sim 40 \%$ of the total signal from the 11 slices, because of the much higher weights associated with those higher redshift slices.

Since we have used a line-of-sight integration limit of $60 h^{-1} \mathrm{Mpc}$ (roughly twice the size of the slice width) to get $w_{p}$ from $\xi$, the measured $w_{p}$ signal will include galaxy pairs that straddle different slices. This effect is not directly reflected in Equation 26, so the cross-correlation between different slices might require a separate treatment. However, because the galaxy correlation functions vary very slowly and smoothly between adjacent slices (a few per cent on large scales; see the bottom sub-panels of Figure 4), the cross terms are most sensitive to the product of the numbers of galaxies in each pair of slices, which is correctly accounted for in Equation 26.

In particular, for two slices $i$ and $j$, Equation 26 makes the assumption that the $3 \mathrm{D}$ cross-correlation $\xi_{i j}$ is approximately
$\left(N_{i}^{2} \xi_{i}+N_{j}^{2} \xi_{j}\right) /\left(2 N_{i} N_{j}\right)$, while the more correct form should be $\sqrt{\xi_{i} \xi_{j}}$, as the cross-correlation is close to the geometric mean of the two auto-correlation functions $\xi_{i}$ and $\xi_{j}$ (Zehavi et al. 2011). When $\Delta \xi / \xi$ is small, the fractional difference between the two forms is of the order $\sim\left(N_{j}-N_{i}\right)^{2} /\left(N_{i} N_{j}\right) \equiv(\Delta N / N)^{2}$. Moreover, after integrating $\xi$ via Equation (2), the $w_{p}$ signal is always dominated by the $\xi^{s}$ values at $r_{\pi}<30 h^{-1} \mathrm{Mpc}$ at any fixed $r_{p}$. We also construct a mock galaxy sample with a similar redshift distribution and bias evolution as the stellar mass samples in the data, and compare the projected correlation functions measured from our approximation in Equation 26) to that directly from the pair counting described in Section 3.1. We find that the error induced by adopting Equation 26 is no more than $2 \%$ on all scales and is thus negligible in our analysis.

It is important to note that the theoretical predictions for $w_{p}$ and $\Delta \Sigma$ via Equation (26) and (27) are independent of the total number of observed galaxies in each sample, i.e., the normalisation of the observed stellar mass function - if we analyse only half of the galaxies randomly drawn from the entire bright 0 sample, the prediction from the same set of model parameters would not change, and the constraints on the model parameters would stay the same, albeit with larger uncertainties.

\subsection{Predicting $w_{p}$ and $\Delta \Sigma$ from a single HOD}

The analytic model for deriving the galaxy clustering and the g-g lensing signals from a single HOD is based on the prescriptions given in a series of papers, including Tinker et al. (2005), Zheng \& Weinberg (2007), and Yoo et al. (2006), with improved treatment 
of the scale-dependent bias, halo exclusion effect, over/underconcentrated satellite distributions from earlier works (e.g. Berlind \& Weinberg 2002, Guzik \& Seljak 2002, Mandelbaum et al. 2006). We describe the prescription briefly below and refer the interested readers to the aforementioned three papers for details.

The signals of $w_{p}$ and $\Delta \Sigma$ are obtained by projecting the $3 \mathrm{D}$ real-space galaxy auto-correlation function $\xi_{g g}$ and the galaxymatter cross-correlation function $\xi_{g m}$, respectively. The projection of $\xi_{g g}$ to $w_{p}$ is given by Equation (2), while for the g-g lensing it is via

$$
\Delta \Sigma\left(r_{p}\right)=\left\langle\Sigma\left(<r_{p}\right)\right\rangle-\Sigma\left(r_{p}\right)
$$

where

$$
\Sigma\left(r_{p}\right)=\bar{\rho}_{m} \int_{-\infty}^{+\infty}\left[1+\xi_{g m}\left(r_{p}, r_{\pi}\right)\right] \mathrm{d} r_{\pi}
$$

and

$$
\left\langle\Sigma\left(<r_{p}\right)\right\rangle=\frac{2}{r_{p}^{2}} \int_{0}^{r_{p}} r_{p}^{\prime} \Sigma\left(r_{p}^{\prime}\right) \mathrm{d} r_{p}^{\prime}
$$

We are ignoring the effects from the radial window, which is broad enough that it is not relevant at galaxy scales (Baldauf et al. 2010). The formalisms for predicting $\xi_{g m}$ and $\xi_{g g}$ are very similar, as the satellite distribution is merely a discrete realisation of NFW tracers with a different concentration than the dark matter. There is, however, one extra contribution to $\xi_{g m}$ and $\Delta \Sigma$ at small scales from the matter retained by the subhalos that host the satellite galaxies (beside the model-independent stellar mass component; see Equation 25). We will discuss this "subhalo" lensing term in more detail in Section 5.3 and focus on the similar terms shared by $\xi_{g g}$ and $\xi_{g m}$ here.

Let us consider a general scenario in which the correlation is between a primary galaxy catalogue and a secondary catalogue consisting of tracer particles $t$, whether it be the dark matter $\left(\xi_{g m}\right)$ or the same galaxies as the primaries $\left(\xi_{g g}\right)$. The correlation signal between the primary and the secondary can be decomposed into two components,

$$
\xi_{g t}(r)+1=\left[\xi_{g t}^{1 h}(r)+1\right]+\left[\xi_{g t}^{2 h}(r)+1\right],
$$

where " $1 \mathrm{~h}$ " and " $2 \mathrm{~h}$ " refer to the so-called "one-halo" and "twohalo" terms, respectively, and the +1 following each $\xi_{g t}$ term is to relate the correlation piece to the corresponding number counts. The " 1 h" term can be theoretically derived via the simple Peebles \& Hauser (1974) estimator,

$$
\xi_{g t}^{1 h}(r)+1=\frac{D D^{1 h}(r)}{R R(r)} .
$$

The $R R(r)$ term is the expected number of pairs consisting of randomly-distributed galaxies and $t$ particles separated by distance between $r$ and $r+\mathrm{d} r$,

$$
R R(r)=g\left(4 \pi r^{2} \mathrm{~d} r\right) \bar{n}_{g} \bar{\rho}_{t} V,
$$

where $\bar{n}_{g}$ and $\bar{\rho}_{t}$ are the mean densities of sample galaxies and $t$ particles, respectively, and $V$ is the survey volume. The prefactor $g$ is 1 if $t$ is a different species than the primary galaxies $\left(\xi_{g m}\right.$, with $\left.\bar{\rho}_{t} \equiv \bar{\rho}_{m}\right)$, and $1 / 2$ if the two are identical $\left(\xi_{g g}\right.$, with $\left.\bar{\rho}_{t} \equiv \bar{n}_{g}\right)$. The $D D(r)$ term requires separate treatment of central and satellite galaxies within each halo first, and then integration over the halo mass function,

$$
\begin{array}{r}
D D^{1 h}(r)=V \mathrm{~d} r \int \frac{\mathrm{d} n}{\mathrm{~d} M_{h}}\left[D D_{\text {cen }, t}\left(M_{h}\right) F_{\text {cen }, t}^{\prime}\left(r \mid c_{t}\right)\right. \\
\left.+D D_{\text {sat }, t}\left(M_{h}\right) F_{\text {sat }, t}^{\prime}\left(r \mid c_{g}, c_{t}\right)\right] \mathrm{d} M_{h},
\end{array}
$$

where $D D_{\text {cen }, t}$ and $D D_{\text {sat }, t}$ are the total numbers of the cen- $t$ and sat- $t$ type of pairs expected within a halo of mass $M_{h}$, respectively, and $F_{\text {cen }, t}(r)$ or $F_{\text {sat }, t}(r)$ is the cumulative probability distribution of the numbers of each of these two pair types within that halo (although Equation (34) requires their respective differential forms, described further below). For $\xi_{g g}$,

$$
D D_{\text {cen }, g}\left(M_{h}\right)=\left\langle N_{\text {cen }}\left(M_{h}\right) N_{\text {sat }}\left(M_{h}\right)\right\rangle,
$$

and

$$
D D_{\text {sat }, g}\left(M_{h}\right)=\left\langle N_{\text {sat }}\left(M_{h}\right)\left(N_{\text {sat }}\left(M_{h}\right)-1\right)\right\rangle / 2,
$$

while $F_{\text {cen }, g}^{\prime}(r)$ and $F_{\text {cen }, g}^{\prime}(r)$ are the galaxy NFW profile of concentration $c_{g}\left(M_{h}\right)$ and the convolution of that galaxy NFW profile with itself, respectively. Similarly for $\xi_{g m}$,

$$
D D_{\text {cen }, m}\left(M_{h}\right)=\left\langle N_{\text {cen }}\left(M_{h}\right)\right\rangle M_{h},
$$

and

$$
D D_{\text {sat }, m}\left(M_{h}\right)=\left\langle N_{\text {sat }}\left(M_{h}\right)\right\rangle M_{h},
$$

while $F_{\text {cen }, m}^{\prime}(r)$ and $F_{\text {sat, } m}^{\prime}(r)$ are the dark matter NFW profile of concentration $c_{d m}\left(M_{h}\right)$ and the convolution of that dark matter profile with the galaxy NFW profile of concentration $c_{g}\left(M_{h}\right)$, respectively. We use the analytic formula in Zheng \& Weinberg (2007) for the convolution of two NFW profiles, either with the same or different concentration parameters. All the $F(r)$ functions are normalised so that $F\left(r=2 r_{200 m}\right)$ is unity, i.e., we assume both the satellite galaxies and dark matter are contained within the virial radii of their host halos. Recently, the study by van Daalen \& Schaye (2015) suggested that it is important to account for the matter outside halos when estimating the small scale matter power spectrum using the halo model. However, this missing power problem does not exist for predicting galaxy clustering and g-g lensing, as the (sub)halos occupied by galaxies are generally massive enough to be fully accounted in the calculations.

The "two-halo" terms are relatively straightforward to calculate above the halo exclusion scales $\left(r_{\mathrm{ex}} \sim 3 h^{-1} \mathrm{Mpc}\right.$, described further below), via

$$
\xi_{g g}^{2 h}(r)=b_{g}^{2} \zeta^{2}(r) \xi_{m m}(r),
$$

and

$$
\xi_{g m}^{2 h}(r)=b_{g} \zeta(r) r_{\mathrm{cc}}(r) \xi_{m m}(r)
$$

where $b_{g}$ is the galaxy linear bias, $\zeta(r)$ characterises the fractional scale-dependence in the large scale halo bias, and $r_{\mathrm{cc}}(r)$ is the cross-correlation coefficient between the galaxies and the dark matter on relevant scales, defined by

$$
r_{c c}(r)=\frac{\xi_{g m}(r)}{\sqrt{\xi_{g g}(r) \xi_{m m}(r)}} .
$$

To a good approximation $r_{\mathrm{cc}}(r)$ is close to unity on scales above $r_{\text {ex }}$ (Guzik \& Seljak 2001; Weinberg et al. 2004, Baldauf et al. 2010), and so we set $r_{\mathrm{cc}}(r) \equiv 1$ and use the empirical fitting function of $\zeta(r)$ from Tinker et al. (2005). We computed $b_{g}$ as the galaxy occupation number-weighted halo bias,

$$
b_{g}=\bar{n}_{g}^{-1} \int b\left(M_{h}\right) \frac{\mathrm{d} n}{\mathrm{~d} M_{h}} \mathrm{~d} M_{h},
$$


where $b\left(M_{h}\right)$ is the halo bias function (see the sixth column of Table 1 for the average linear bias of each iHOD sample).

Within the halo exclusion regime (Cacciato et al. 2009), however, Equations (39) and (40) are no longer valid because the centre of one halo cannot sit within the virial radius of another halo, i.e., $r_{\text {ex }}<2 \times r_{200 \mathrm{~m}}^{\max }$, where $r_{200 \mathrm{~m}}^{\max }$ is the maximum halo radius in the calculation. Therefore, the "two-halo" terms at a given distance require explicit integration over pairs of halos that are too small to run into the centre of each other when separated by that distance. We follow the prescription described in Tinker et al. (2005) for the treatment of halo exclusion and adopt the method in Yoo et al. (2006) to circumvent the issue of unsatisfied integral constraints in the halo mass function and halo bias function (see Equation 16 of Yoo et al.2006 for details).

\subsection{Subhalo Contribution to $\Delta \Sigma$ in MassiveBlack-II}

The extra "subhalo" lensing term, as mentioned in Section 5.2 arises because the satellite galaxies are sitting at the local density peaks (i.e., their subhalos), rather than random positions within their main halos. From investigating a suite of N-body cosmological simulations, Mandelbaum et al. (2005) found that the fractional contribution of the subhalo term on scales below $0.1 h^{-1} \mathrm{Mpc}$ is roughly equal to the satellite fraction within the sample, but is cut off on slightly larger scales because of the tidal truncation of those subhalos inside larger halos. They discovered that a truncation of the subhalos at $\sim 0.4$ times the virial radii gives a good match to the lensing signal measured from the simulations. A subsequent exploration by Yoo et al. (2006), however, estimated that the error induced by ignoring the subhalo contribution is below $10 \%$ on the relevant scales using a suite of relatively small Smoothed Particle Hydrodynamic (SPH) simulations. It is still controversial as to whether the modelling of the subhalo lensing is necessary. While some studies (Velander et al. 2014, Hudson et al.|2015) adopted the "tidally stripped" subhalo lensing model proposed in Mandelbaum et al. (2005), other studies (e.g., Leauthaud et al. 2012b, Coupon et al.2015) ignored the lensing from subhalos, either based on the result of Yoo et al. (2006), and/or the fact that the measured total lensing signal is much less constraining than other probes used in their papers (SMF and galaxy clustering).

We revisit the subhalo lensing issue by employing the MassiveBlack-II ${ }^{4}$ SPH simulation (MB-II; Khandai et al. 2014), a P-GADGET cosmological hydrodynamic simulation evolved with a total of $2 \times 1792^{3}$ dark matter and gas particles (mass resolution $\sim$ several times $10^{6} h^{-1} M_{\odot}$ ) inside a cubic volume of $100^{3} h^{3} \mathrm{Mpc}^{-3}$. MB-II is one of the highest resolution simulations of this size which includes a self-consistent model for star formation, black hole accretion and associated feedback. Thanks to its exquisite capability of resolving subhalos down to $10^{9} h^{-1} M_{\odot}$ with realistic baryonic effects (for the details on the HOD of simulated galaxies in MB-II, see Tucker et al, in prep), we quantify the impact of the often-ignored subhalo lensing term, by comparing the $g$ - $g$ lensing signals measured from the MB-II galaxies with subhalos and from a sample of mock galaxies without. Following Yoo et al. (2006), in order to construct a subhalo-less mock galaxy sample, we identify the satellite galaxies in each main halo, and randomise their position angles relative to the halo centre while keeping their halo-centric distances fixed. In this way, we ensure that the only difference between the lensing signals measured from the

4 http://mbii.phys.cmu.edu/
MB-II galaxies and the mock galaxies on small scales is induced by the presence vs. absence of dark matter within the subhalos.

Figure 5 shows the results of this experiment in six different stellar mass bins at $z=0.06$, with $M_{*}$ increasing from left to right and top to bottom. In each top sub-panel, the satellite fraction and the stellar mass range are marked on the top. The open circles with errorbars indicate the measurement for the total g-g lensing signal for that stellar mass bin, decomposed into the central (red solid) and satellite (blue solid) terms. The errorbars are derived from jackknife resampling of the simulation volume. The cyan dashed line shows the satellite term measured from the mock galaxies, which decreases rapidly on small scales due to the lack of subhalos. The magenta dashed line is a crude estimate of the subhalo lensing term via re-scaling the amplitude and truncating the central term (red solid) at $0.4 r_{200 \mathrm{~m}}$. The amplitude is re-scaled by $f_{\text {sat }} /\left(1-f_{\text {sat }}\right)$, assuming the subhalos that host satellites share the same inner matter density profile as those host central galaxies of the same stellar mass, analogous to the ansatz employed in the SHAM technique. The black solid line is the sum of this estimated subhalo term, the directly measured central term, and the satellite term from the mock sample, serving as a rough estimate of the predicted total signal with the subhalo lensing contribution, whereas the black dashed line is the sum of central and mock satellite terms without any subhalo contribution. The difference between the open circles and the black dashed lines represents the magnitude of the error on g-g lensing due to ignoring the subhalo lensing piece, and the difference between the open circles and the black solid lines represents the deficiency of the overly-simplified "re-scaled central" subhalo lensing model in over-predicting or under-predicting the signal at various scales.

The effects of ignoring the subhalo lensing term are better illustrated by the bottom sub-panels of Figure 5 where the black solid and dashed lines shown above are divided by the measured total signals. The gray shaded region indicates the typical uncertainties on the ratios propagated from the measurement uncertainties on $\Delta \Sigma$. The ratio plots clearly demonstrate that, ignoring the subhalo lensing term causes $15 \%-35 \%$ systematic under-prediction of the total signal on scales below $\sim 0.2 h^{-1} \mathrm{Mpc}$, and the deviation is proportional to the fraction of satellite galaxies in the sample. This contradicts the result from Yoo et al. (2006) who found the effect generally below $10 \%$ at all radius. The opposite conclusions drawn from the two sets of simulations could be due to the drastic difference in the resolution and size - MBII has 2000 times more particles in 8 times the volume of the simulation used by Yoo et al. (2006), and/or the average mass of the subhalos used in the two experiments (i.e., Yoo et al. 2006 could only go to subhalos above $10^{12} h^{-1} M_{\odot}$, hence much lower satellite fraction). Once the simple "re-scaled central" subhalo lensing term is added, the predicted signal agrees with the direct measurement within the uncertainties for the three high stellar mass bins in the bottom row, but overpredict the total signal by $15 \%-35 \%$ on the relevant scales for the three low stellar mass bins in the top row. This discrepancy between the simple remedy and the simulation in the low mass samples is caused by the enhanced tidal attenuation effect on the low mass subhalos from their host halos, besides the usual tidal truncation effect seen for subhalos of all masses. This attenuation effect is consistent with the findings in Li et al. (2014), who measured the subhalo lensing signal using the satellite lens galaxies selected from the SDSS group catalogue constructed from a redshift-space Friends-of-Friends algorithm (Yang et al. 2007). They found significant $\Delta \Sigma$ signal below projected radius $\sim 0.1 h^{-1} \mathrm{Mpc}$ for satellite lenses located $0.1-0.5 h^{-1} \mathrm{Mpc}$ away from their respective group 

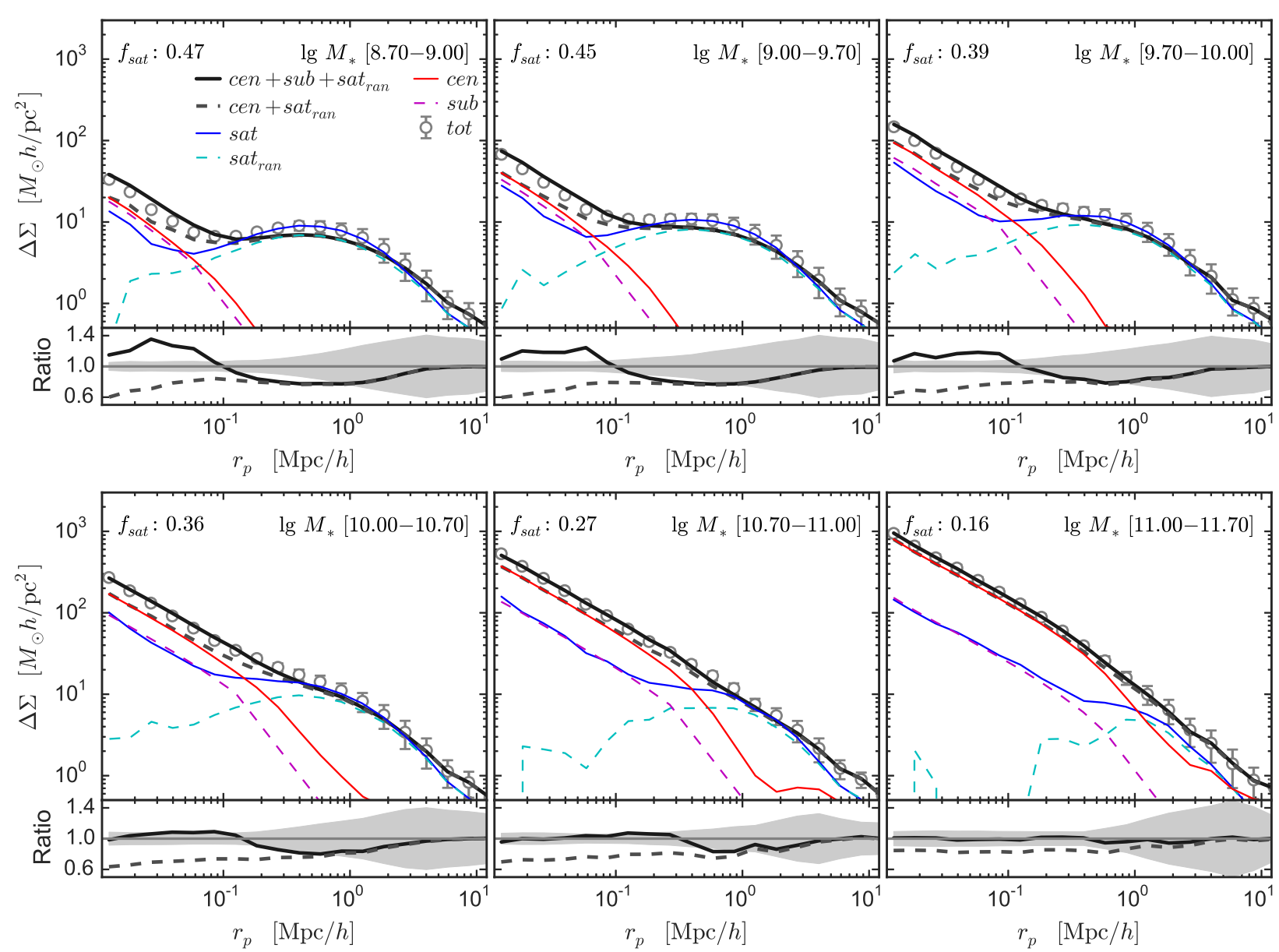

Figure 5. The g-g lensing signal of six different stellar mass samples in the MB-II simulation at $z=0.06$, highlighting the importance of including the subhalo contribution in the modelling of the surface density contrast $\Delta \Sigma$. In each upper sub-panel, open circles are the total $\Delta \Sigma$ signal measured directly from the simulation, which is the sum of the red and the blue solid curves, indicating the contributions from the centrals and the satellites, respectively. The cyan dashed curve represents the satellite contribution measured after randomising the position angles of the simulated satellites (distances to the halo centre kept fixed). The magenta dashed curve accounts for the subhalo contribution by re-scaling the central contribution according to the satellite fraction listed on the top left and truncating the 3D density profile at $0.4 r_{200 \mathrm{~m}}$. The black solid curve is the sum of the subhalo (magenta), the central (red), and the randomised satellite (cyan) curves, and the black dashed curve the sum of only the latter two. The ratio of the black solid and the black dashed curves to the open circles is shown on each lower sub-panel. Ignoring the subhalo contribution results in an underestimate of the total signal by $15 \%-35 \%$ on small scales, roughly proportional to the satellite fraction in each sample. For the three low mass samples, using the re-scaled and truncated central curve as a rough estimate for the subhalo contribution overestimates the total signal by $10 \%-35 \%$ on small scales, and by $\sim 15 \%$ in the transitional regime, albeit within the measurement uncertainties indicated by the gray band. For the high mass samples the black curves agree with the direct measurements within the uncertainties across all scales.

centres, and the amplitude of the subhalo lensing signal can be explained by a truncated and attenuated version of those NFW halos that host central galaxies of the same stellar masses.

Drawing from these findings in the MB-II simulation and the SDSS groups, we model the subhalo contribution to the galaxymatter cross-correlation function $\xi_{g m}^{\text {sub }}$ as an attenuated and truncated version of the central term $\xi_{g m}^{\mathrm{cen}}$,

$$
\xi_{g m}^{\mathrm{sub}}\left(r \mid M_{*}\right)= \begin{cases}f_{t} \times\left[\frac{f_{\mathrm{sat}}}{1-f_{\mathrm{sat}}} \xi_{g m}^{\mathrm{cen}}\left(r \mid M_{*}\right)\right], & r<r_{t} \\ 0, & r \geqslant r_{t},\end{cases}
$$

where $f_{t}$ and $r_{t}$ is the attenuation factor and the truncation radius, respectively. We adopt a truncation radius of $r_{t}=0.4 r_{200 m}\left(M_{*}\right)$, according to the findings in Mandelbaum et al. (2006) and Figure 5 The value of $f_{t}$ should be stellar mass dependent - we adopt $f_{t}$ values of 0.5 and 1.0 for stellar mass samples below and above $10^{10} h^{-2} M_{\odot}$, respectively, informed by the results in Figure 5
However, as mentioned in Section 4.4 , the small-scale lensing modelling is further complicated by the miscentering effect, which is absent from Figure 5 As pointed out in Yoo et al. (2006), the miscentering effect smooths the overall $\Delta \Sigma$ signal on small scales with a kernel of length $\sim 0.1 h^{-1} \mathrm{Mpc}$, and is likely to have a large impact for the higher stellar mass bins, i.e., within groups and clusters. The smoothing effect will effectively reduce the predicted signal for the central galaxies at the $10 \%-15 \%$ level on scales below $0.1 h^{-1} \mathrm{Mpc}$, where the subhalo lensing effect operates in the opposite direction. Since the satellite fraction at the high mass bins is $\sim 20 \%$, this reduction can be effectively absorbed by a low $f_{t}$ value of $\sim 0.5$ in Equation 43 for the high mass bins. Therefore, to simultaneously capture both the effects of subhalo lensing at low $M_{*}$ and miscentering at high $M_{*}$ on scales below $0.1 h^{-1} \mathrm{Mpc}$, we adopt a constant attenuation factor of $f_{t}=0.5$ in our analysis, regardless of the stellar mass of the sample. We have tried different 
values of $f_{t}=0.5 \pm 0.2$ and found that our results are robust to those changes, due to the relatively large statistical uncertainties of the g-g lensing measurements on small scales (see Figure 6).

For each redshift slice within the $i$ HOD samples and for each individual $\mathrm{CHOD}$ sample, the predictions for the $w_{p}$ and $\Delta \Sigma$ signals are obtained by projecting $\xi_{g g}$ and $\xi_{g m}$ to $2 \mathrm{D}$, according to Equation (2) and (28), respectively. We adopt the same $r_{\pi}^{\max }=$ $60 h^{-1} \mathrm{Mpc}$ for the theoretical predictions of $w_{p}$ as for the measurements. Finally, for each $i$ HOD sample we combine the predictions from all its redshift slices to obtain the predictions for that entire sample via Equations (26) and 27).

Figure 6 compares the predictions from our best-fit model (thick lines) to the measurements from data for the eight $i$ HOD stellar mass samples. The galaxy clustering and g-g lensing results are shown in each pair of the top and bottom subpanels, respectively. Each best-fit curve is decomposed into the two-halo (thin dot-dashed), the one-halo central (thin dashed), and the one-halo satellite (thin solid) contributions. For the best-fit gg lensing signal of each sample, we also show the contributions from the subhalo lensing term and the "point source" stellar mass as the thin dotted and dot-dot-dashed lines, respectively. The subhalo lensing term begins to dominate the one-halo satellite contribution at around half the virial radius of the average main halo corresponding to that sample, and then the stellar mass term takes over at the galactic scales below tens of $h^{-1} \mathrm{kpc}$. We will come back to Figure 6 for the detailed comparison between the model fits and the measurements in Section 6.2

\section{PARAMETER CONSTRAINTS}

\subsection{Likelihood Model and Bayesian Inference}

Armed with the capability of predicting the galaxy clustering and g-g lensing signals for any given $C H O D$ or $i$ HOD sample, we can infer the posterior probability distributions of the model parameters from the measurements within a Bayesian framework, assuming a Gaussian likelihood model and a set of uninformative priors on those parameters.

The observable vector in our likelihood model has two components:

1. $w_{p}^{i}\left(r_{p}^{j}\right): w_{p}$ profile of stellar mass sample $i$ measured at projected radius $r_{p}^{j}$ (from $r_{p}^{\mathrm{fb}}$ to $20.0 h^{-1} \mathrm{Mpc}$ ), for $i \in$ $\{1 \cdots 8\}$ (iHOD) or $\in\{1 \cdots 6\}$ (CHOD), and $j \in\{1 \cdots n\}$, where $n$ is the number of data points used for each stellar mass samples. Ranked by ascending order in the average stellar mass, the samples have $n \in\{17,16,15,14,14,13,13,10\}$ (i HOD) or $n \in\{16,15,14,14,13,10\}$ (CHOD), due to the different fibrecollision induced cutoffs on small scales. There are 112 (82) data points in total for the $i$ HOD (CHOD) analysis.

2. $\Delta \Sigma^{i}\left(r_{p}^{k}\right): \Delta \Sigma$ profile of stellar mass sample $i$ measured at radius $r_{p}^{k}$, ranging from $r_{p}^{\text {cut }}$ to $20.0 h^{-1} \mathrm{Mpc}$, where $r_{p}^{\text {cut }}$ is the small-scale lensing cutoff caused by the systematic uncertainties in estimating the "boost factor" for bright samples (see Appendix for more details). We use the same set of stellar mass samples as clustering in each analysis. The minimum fitting scale is $25 h^{-1} \mathrm{kpc}$ for the samples below $\lg M_{*}=10.6$, but increases as the sample gets brighter due to large $r_{p}^{\text {cut }}$, reaching $0.12 h^{-1} \mathrm{Mpc}$ for the highest stellar mass bin. Therefore, $k \in$ $\left\{1 \cdots n^{\prime}\right\}$, where $n^{\prime} \in\{18,18,18,18,17,17,16,15\}$ (i HOD) and $\in\{18,18,17,17,16,15\}$ (CHOD). There are 137 (101) $\Delta \Sigma$ data points in total for the $i \mathrm{HOD}$ (CHOD) analysis.
We model the combinatorial vector $\mathbf{x}$ of the $w_{p}$ and the $\Delta \Sigma$ components as a multivariate Gaussian $(N=256$ variables in total for $i$ HOD and $N=190$ for $C H O D$ ), which is fully specified by its mean vector $(\overline{\mathbf{x}})$ and covariance matrix $(C)$. The Gaussian likelihood is thus

$$
\mathcal{L}(\mathbf{x} \mid \boldsymbol{\theta})=|C|^{-1 / 2} \exp \left(-\frac{(\mathbf{x}-\overline{\mathbf{x}})^{T} C^{-1}(\mathbf{x}-\overline{\mathbf{x}})}{2}\right),
$$

where

$$
\begin{aligned}
\boldsymbol{\theta} \equiv\left\{\lg M_{h}^{1}, \lg M_{*}^{0}, \beta, \delta, \gamma, B_{\text {sat }},\right. \\
\left.\beta_{\text {sat }}, B_{\text {cut }}, \beta_{\text {cut }}, \alpha_{\text {sat }}, \sigma_{\ln M_{*}}, \eta, f_{c}\right\} .
\end{aligned}
$$

We adopt flat priors on the model parameters, with a uniform distribution over a broad interval that covers the entire possible range of each parameter (see the 3rd column of Table 2). The final covariance matrix $C$ is assembled by aligning the error matrices of $w_{p}$ and $\Delta \Sigma$ measured for individual samples along the diagonal blocks of the full $N \times N$ matrix. We ignore the weak covariance between $w_{p}$ and $\Delta \Sigma$ (with the covariance being weak due to the fact that $\Delta \Sigma$ is dominated by shape noise), and between any two measurements of the same type but for different stellar mass samples.

In the parameter inference stage, the posterior distribution is derived using the Markov Chain Monte Carlo (MCMC) algorithm emcee (Foreman-Mackey et al. 2013), where an affine-invariant ensemble sampler is utilised to fully explore the 13-D parameter space. For each MCMC chain of the iHOD and CHOD inferences, we perform 90,000 iterations, 30,000 of which belong to the burn-in period for adaptively tuning the steps. To eliminate the tiny amount of residual correlation between adjacent iterations, we further thin the chain by a factor of 10 to obtain our final results.

\subsection{Posterior Probability Distributions}

Figure 7 presents a summary of the inferences for both the iHOD (brown filled) and CHOD (gray open) analyses, showing the $1 \mathrm{D}$ posterior distribution for each of the 13 model parameters (diagonal panels), and the $95 \%$ and $68 \%$ confidence regions for all the parameter pairs (off-diagonal panels). In the panels of the lower triangle, we highlight the results from our fiducial model, i.e., the $i$ HOD model, employing the clustering and g-g lensing from the entire galaxy population above the mixture limit and self-consistently accounting for the survey incompleteness in stellar mass. In those panels we also show the $95 \%$ confidence regions from the $i \mathrm{HOD}$ constraints that employ the clustering and the lensing signals separately. The constraint from using the clustering alone (blue solid contours) is significantly tighter than that from the g-g lensing (red dashed contours), due to the much higher $\mathrm{S} / \mathrm{N}$ in the $w_{p}$ measurements. However, the g-g lensing is absolutely essential in the joint iHOD analysis, helping break the degeneracy between the scatter parameters $\left(\sigma_{\ln M_{*}}\right.$ and $\left.\eta\right)$ and the slope of the SHMR on the high mass end $(\delta)$. In each panel of the upper triangle, we compare the constraints from the fiducial model (filled contours) to that of the traditional approach, i.e., the CHOD model, which is limited to $54 \%$ of the iHOD galaxies and assumes the samples to be complete (open contours). While the two constraints are largely consistent with each other, the $I$ HOD analysis is able to obtain a much tighter constraint because of the larger span in stellar mass range and the higher number of galaxies (hence the higher $S / N$ of measurements illustrated in Figure 2). In particular, the iHOD analysis substantially improves the constraints on the pivot of the SHMR 

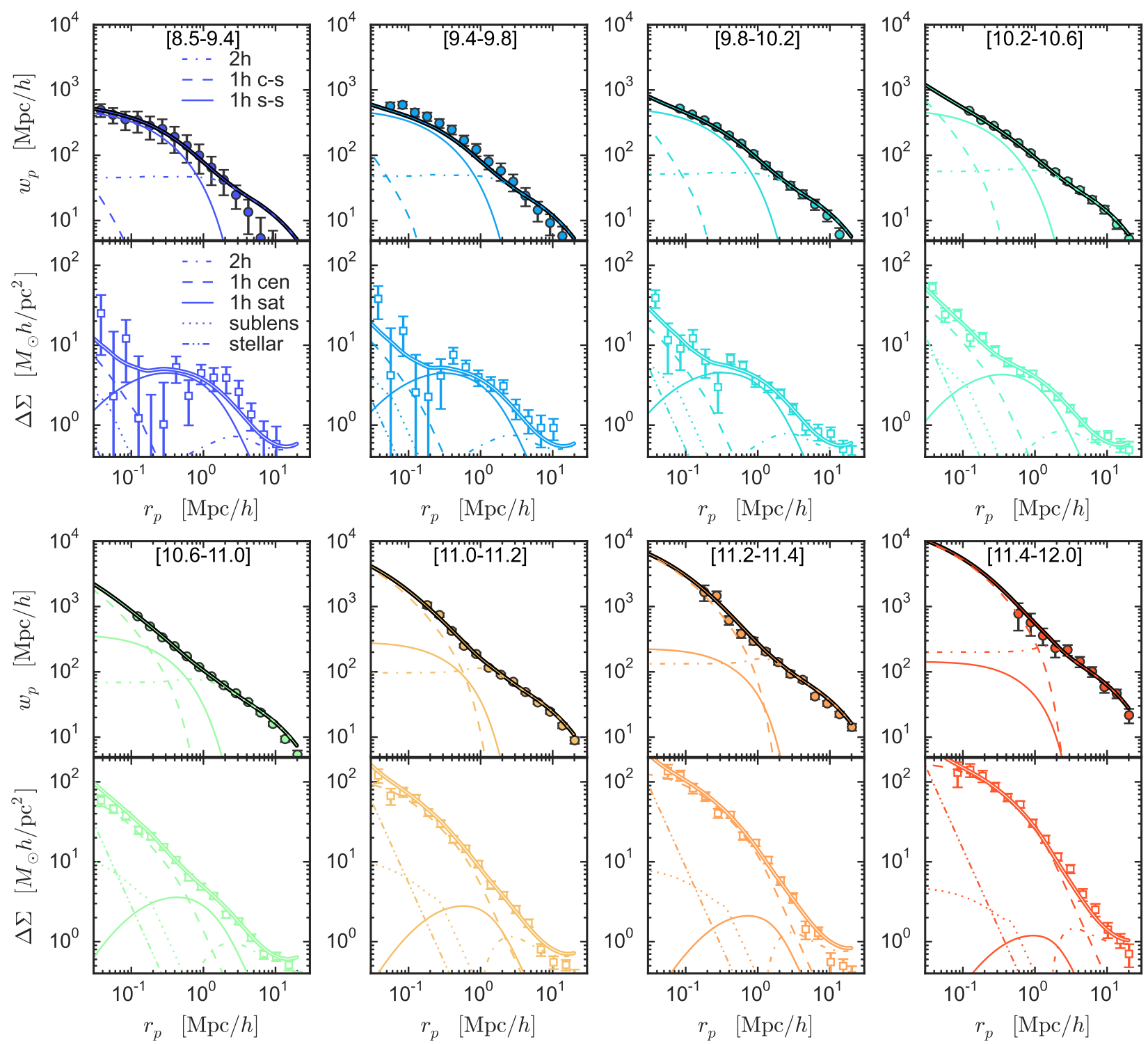

Figure 6. Comparison between the galaxy clustering and g-g lensing measurements from SDSS with the signals predicted by the best-fit i HOD model, for the eight stellar mass-selected samples. For each sample, the top and bottom panels show the projected correlation function $w_{p}$ and g-g lensing signal $\Delta \Sigma$, respectively. In each panel, the data points with errorbars are the measurements, and the thick line is the best-fit signal, which is decomposed into the 2-halo (thin solid), the 1-halo central (thin dashed), and the 1-halo satellite (thin dot-dashed) contributions shown underneath. The dotted and the dot-dotdashed lines in the lower sub-panels represent the lensing contributions from the subhalo dark matter and the galaxy stellar mass, respectively. The $\mathrm{x}$-/y-axis ranges are uniform across all panels. The model provides excellent fit to both the clustering and lensing signals of galaxies over four decades in stellar mass.

$\left(\lg M_{h}^{1}, \lg M_{*}^{0}\right)$ and its high-mass end slope $\delta$. The five parameters that describe the satellite HOD benefit the most from the inclusion of low stellar mass samples, with much stronger constraint in $i$ HOD than in CHOD. The $68 \%$ confidence regions of the $1 \mathrm{D}$ posterior constraints are listed in Table 2

The iHOD model favours a scatter of $\sigma_{\ln M_{*}}=0.50_{-0.03}^{+0.04}$ about the mean SHMR with a slightly negative slope of $\eta=$ $-0.04 \pm 0.02$, while the $\mathrm{CHOD}$ model infers a constant scatter of $\sigma_{\ln M_{*}}=0.42_{-0.08}^{+0.07}$. However, the two constraints converge to a similar scatter on the high mass end, where the $i$ HOD model shrinks its mass-dependent scatter to meet the lower constant value preferred by the $C H O D$ model. While the $C H O D$ does not constrain the concentration ratio $f_{c}$ well, the iHOD strongly favours the scenario where the satellite distributions are $\sim 15 \%$ less concentrated than the dark matter within the same halos $\left(f_{c}=0.86_{-0.11}^{+0.14}\right)$. This galaxy under-concentration agrees with observational findings in Wang et al. (2014) and Budzynski et al. (2012) (but see Watson et al.2012 for the degeneracy between $f_{c}$ and the inner slope of the cluster density profiles).

The improvement on the overall constraints from 1 HOD to CHOD is better illustrated in Figure 8, where we translate the uncertainties of the individual model parameters to that of the mean SHMR and the satellite HOD separately. In the left panel, the red solid and gray dashed curves are the mean SHMR inferred from the iHOD and CHOD analyses, respectively. The width of each shaded band, i.e., the $68 \%$ uncertainty in the mean log-stellar mass at fixed halo mass, directly reflects the joint posterior probability distribution of the five parameters $\left(\lg M_{h}^{1}, \lg M_{*}^{0}, \beta, \delta, \gamma\right)$ that determine 


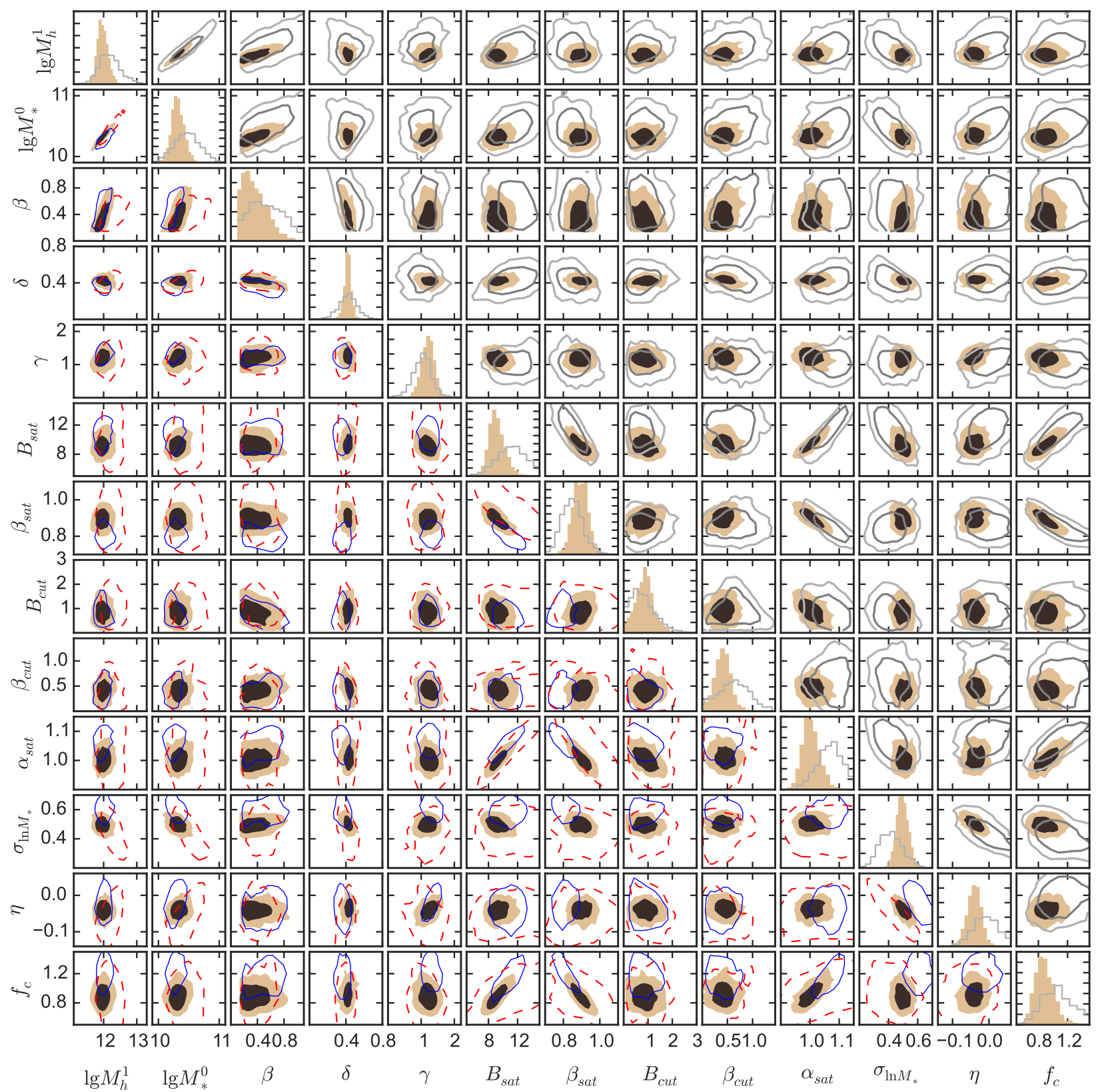

Figure 7. Confidence regions from our $i$ HOD analysis of the galaxy clustering and the g-g lensing data in the 2D planes that comprised of all the pair sets of model parameters. Histograms in the diagonal panels show 1D posterior distributions of individual parameters. For comparison, constraints from the $\mathrm{CHOD}$ analysis are only shown in the panels of the upper triangle. Contour levels run through confidence limits of $95 \%$ (light brown/gray) and $68 \%$ (dark brown/gray) inwards. The tighter $i$ HOD constraint compared to CHOD is due to the combination of improved overall statistics from larger samples and additional information from the low mass galaxies. In the panels of the lower triangle, blue solid and red dashed contours indicate the $95 \%$ confidence regions of the two separate iHOD constraints using the clustering and the lensing data, respectively.

the mean SHMR. The two constraints are consistent with each other, especially on the high mass end. This is very reassuring — as we mentioned in Section 4.1 the two models are identical to each other for the high stellar samples, because the completeness approaches unity and is independent of halo mass. As expected from Figure 7, the uncertainties in the mean SHMR are greatly reduced across all halo masses in the $i$ HOD constraint, with the most significant reduction happening above $M_{h}=10^{13} h^{-1} M_{\odot}$ (by factor of two in log-stellar mass). Furthermore, both of the mean SHMRs are best constrained at the intermediate mass range around the piv- otal point $\left(\lg M_{h}^{1}, \lg M_{*}^{0}\right)$, due to the highest model sensitivity and the highest $S / N$ at this stellar mass.

Similarly, in the right panel of Figure 8 we show the constraints on the satellite occupation number predicted for five of the stellar mass samples that were used in the $\mathrm{CHOD}$ analysis. The gray dashed lines show the median satellite occupation numbers as functions of halo mass inferred from the $\mathrm{CHOD}$ analysis, with their 1- $\sigma$ uncertainties shown as the corresponding gray bands. For comparison, we have predicted two types of satellite HODs for these five CHOD samples from the best-fit iHOD model, one is for the par- 

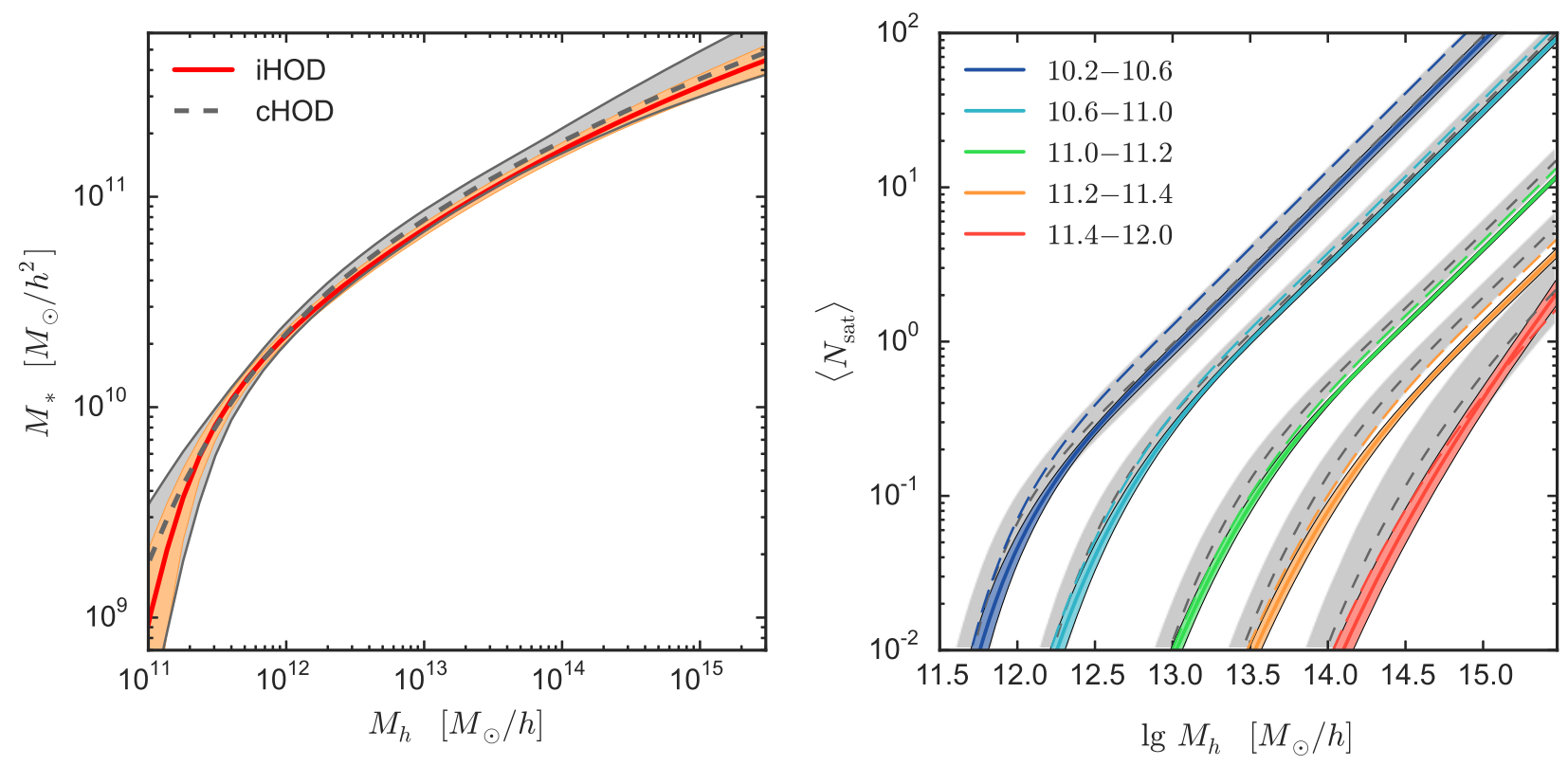

Figure 8. Constraints on the SHMR for the central galaxies (left) and the satellite HODs for four stellar mass bins (right), as a more visually appealing and scientifically informative presentation of the confidence regions shown in Figure 7 In the left panel, the red solid and the gray dashed lines show the inferred SHMR from the $i$ HOD and CHOD analysis, respectively, and the shaded regions represent the $68 \%$ confidence range at fixed halo mass. In the right panel, solid coloured and dashed gray lines show the expected HODs for the observed galaxies from the best-fit $\mathrm{H}$ HOD and CHOD model, respectively, with each shaded band indicating the $68 \%$ confidence range at fixed halo mass; long-dashed coloured lines show the parent HODs predicted from the best-fit $i$ HOD model. The difference between the solid and long-dashed coloured lines implies possible incompleteness in the stellar mass samples.

Table 2. Description, prior specifications, and posterior constraints of the parameters in the model. All the priors are uniform distributions running across the entire range of possible values for the parameters, and the uncertainties are the $68 \%$ confidence regions derived from the 1D posterior probability distributions.

\begin{tabular}{ccccc}
\hline \hline Parameter & Description & Uniform Prior Range & iHOD & CHOD \\
\hline $\lg M_{h}^{1}$ & Characteristic halo mass of the SHMR & {$[9.5,14.0]$} & $12.10_{-0.14}^{+0.17}$ & $12.32_{-0.29}^{+0.29}$ \\
$\lg M_{*}^{0}$ & Characteristic stellar mass of the SHMR & {$[9.0,13.0]$} & $10.31_{-0.09}^{+0.10}$ & $10.47_{-0.21}^{+0.18}$ \\
$\beta$ & Low-mass slope of the SHMR & {$[0.0,2.0]$} & $0.33_{-0.15}^{+0.21}$ & $0.54_{-0.26}^{+0.29}$ \\
$\delta$ & Controls high-mass slope of the SHMR & {$[0.0,1.5]$} & $0.42_{-0.04}^{+0.03}$ & $0.42_{-0.09}^{+0.08}$ \\
$\gamma$ & Controls intermediate-mass behaviour of the SHMR & {$[-0.1,4.9]$} & $1.21_{-0.20}^{+0.18}$ & $1.05_{-0.26}^{+0.24}$ \\
$B_{\text {sat }}$ & Normalises the scaling of $M_{\text {sat }}$ & {$[0.01,25.0]$} & $8.98_{-0.87}^{+1.18}$ & $11.22_{-1.99}^{+2.61}$ \\
$\beta_{\text {sat }}$ & Slope of the scaling of $M_{\text {sat }}$ & {$[0.1,1.8]$} & $0.90_{-0.05}^{+0.04}$ & $0.85_{-0.05}^{+0.06}$ \\
$B_{\text {cut }}$ & Normalises the scaling of $M_{\text {cut }}$ & {$[0.0,6.0]$} & $0.86_{-0.37}^{+0.32}$ & $0.73_{-0.44}^{+0.58}$ \\
$\beta_{\text {cut }}$ & Slope of the scaling of $M_{\text {cut }}$ & {$[-0.05,1.50]$} & $0.41_{-0.15}^{+0.16}$ & $0.63_{-0.33}^{+0.31}$ \\
$\alpha_{\text {sat }}$ & Power-law slope of the satellite HOD & {$[0.5,1.5]$} & $1.00_{-0.02}^{+0.03}$ & $1.07_{-0.05}^{+0.05}$ \\
$\sigma_{\ln M_{*}}$ & Low-mass scatter in the SHMR & {$[0.01,3.0]$} & $0.50_{-0.03}^{+0.04}$ & $0.42_{-0.08}^{+0.07}$ \\
$\eta$ & Slope of the scaling of high-mass scatter & {$[-0.4,0.4]$} & $-0.04_{-0.02}^{+0.02}$ & $-0.01_{-0.03}^{+0.04}$ \\
$f_{c}$ & Concentration ratio between satellites and dark matter & {$[0.1,3.0]$} & $0.86_{-0.11}^{+0.14}$ & $1.06_{-0.20}^{+0.32}$ \\
\hline
\end{tabular}

ent satellite galaxies (thin dashed lines), and the other for the $o b$ served galaxies (thick solid lines). To avoid clutter, we only show the uncertainty bands associated with the inferred observed satellite HODs, all of which are considerably narrower than the gray bands, especially for the two highest stellar mass samples. This improvement is very encouraging. As we will show later in Section 7.3 , the occupation statistics of the satellite galaxies, long being regarded as a mere nuance in the HOD modelling of galaxy clustering and HOD-based cosmological constraints (Yoo \& Seljak 2012), is constrained well enough in the $\mathrm{iHOD}$ analysis to provide important insights into the physical formation and evolution of the satellite galaxies.

It is important to point out that, when calculating the HODs for the observed galaxies in Figure 8 we have made use of the actual number of observed galaxies in each sample in order to normalise the amplitude of $\left\langle N_{\text {sat }}\left(M_{h}\right)\right\rangle$ using Equation (12), while the parent HODs are predicted directly from the best-fit model parameters using Equation (9). As mentioned in Section 5.1 the 1 HOD analysis constrains the host halo distribution of the average galaxy in each sample by matching to the observed clustering and g-g lensing signals, and is thus entirely agnostic of the observed amplitude of the stellar mass functions. Therefore, it is highly nontrivial to discover in the right panel of Figure 8 that, all the predicted parent HODs are lying right above their observed counterparts, consistent with our expectation that the galaxy samples above the mixture limit 
are subject to some mild level of incompleteness. We will inspect closely this consistency later in Section 7.1

Figure 6 compares the clustering and g-g lensing signals measured from data to that predicted from the set of best-fit parameters derived from the median values of their corresponding 1D posterior probability distributions. We refer to this set of "median" parameters as the "best-fit" parameters for simplicity, despite that it does not produce the maximum posterior probability or likelihood value. The lowest stellar mass sample is subject to severe cosmic variance due to small volume, resulting in a relatively poor $w_{p}$ fit on large scales. The model also under-predict the small scale clustering signal for the $\lg M_{*}=[9.4,9.8]$ sample, which is largely overrun by the CfA2 great wall (Geller \& Huchra 1989). Overall, the model provides an excellent fit to the data over almost four decades in stellar mass, across distance scales from the galactic disks to tens of Mega-parsecs.

\subsection{Systematic Uncertainties in the iHOD Model}

Observationally, the systematic errors in our fiducial i HOD analysis come from the uncertainties in the stellar mass estimates and in the measurements of the projected correlation functions and the g-g lensing signals. As mentioned in Section 2.2. the estimation of $M_{*}$ is subject to various photometric and model uncertainties that mostly affect the overall normalization of the derived stellar masses, with little perturbation in the ranking order of individual galaxies within the catalogue. Therefore, the inferred mapping between the stellar mass and the dark matter halos from our analysis can be straightforwardly re-calibrated for other stellar mass estimators.

The uncertainties in the $w_{p}$ and the $\Delta \Sigma$ measurements are estimated internally from the data via jackknife resampling. Compared to external estimates derived from multiple independent catalogues, the jackknife estimate somewhat overestimates the correlation function errors on scales below $r_{p} \sim 2 h^{-1} \mathrm{Mpc}$ (Norberg et al. 2009), while on large scales it likely underestimates the errors because it does not include the cosmic variance above the scales of individual subsamples. Therefore, the jackknife errors for the two lowest stellar mass/redshift samples are underestimated on large scales, because of the small physical size of the jackknife patches. The error budget of these two stellar mass samples, however, is still dominated by the uncertainties on small scales, so the fiducial constraint is insensitive to the underestimation in the error covariance on large scales, despite the inadequate fit to the $w_{p}$ measurements of the two lowest stellar samples on relevant scales.

The systematic errors in the $\Delta \Sigma$ measurements have additional contributions from the calibration biases mainly related to the shear estimation and the photo- $z$ errors, each at a few per cent level (see Appendix A for the scale-dependent lensing systematics). Mandelbaum et al. (2013) addressed these systematics by conducting a suite of ratio tests (Mandelbaum et al. 2005), i.e. comparisons of the signal computed using the same lens samples, but with different sub-samples of the source catalogue. After applying well-understood corrections, the systematic errors are subdominant compared to the statistical errors and compared to the systematic uncertainties due to modelling assumptions, which we will discuss next.

The theoretical systematic errors in our fiducial i HOD analysis have four main sources: 1) simplified model assumptions in the iHOD formalism, 2) uncertainties in the theoretical prediction of halo statistics like the halo mass function and the halo bias function, 3) our ansatz of the weak $M_{h}$-dependence of the detection rate at fixed $M_{*}$, and 4) uncertainties in the combined treatment of the mis-centering and subhalo lensing. We have discussed 3) in detail in Section 4.1 and will comment more on its potential impact in Section 7.1 and the impact from 4) has been discussed and addressed in Section 5.3 Therefore, here we focus on the first two issues and discuss their potential impacts on our model constraints in turn.

As a variant of the HOD formalism, the $i$ HOD model inherits the systematic uncertainties associated with the generic HOD models, which assume that the average galaxy content of halos depends solely on the halo mass, predicted by the basic excursion set theory of structure formation (Press \& Schechter 1974, Bardeen et al. 1986. Bond et al. 1991). However, since the halo assembly histories in cosmological simulations are affected by the large-scale environment (Wang et al. 2007, Dalal et al. 2008), some halo properties, including age, concentration, spin, richness, and most importantly clustering, exhibit systematic differences between low and high density environments at fixed halo mass (Sheth \& Tormen 2004, Gao et al.|2005, Wechsler et al.|2006, Harker et al. 2006: Zhu et al. 2006, Hahn et al. 2007, Jing et al. 2007, Li et al. 2008, Faltenbacher \& White 2010, Croft et al. 2012). This effect, broadly termed "halo assembly bias" (Gao \& White 2007), has likely left its imprint on galaxy formation histories as well, resulting in a "galaxy assembly bias". However, hydrodynamic simulations and SAMs predict only a small impact $(<10 \%)$ of the halo assembly bias on galaxy clustering statistics (Yoo et al. 2006, Croton et al. 2007; Zu et al. 2008), while observationally a smoking-gun detection of the galaxy assembly bias remains elusive (Berlind et al. 2006, Blanton \& Berlind 2007, Wang et al. 2013, Hearin et al. 2014). Additionally, the halo assembly bias becomes only significant for halos below the characteristic non-linear mass scale, where our error budget is dominated by statistical errors and cosmic variance. However, it is worth noting that halo assembly bias may have a much greater impact on the HOD modelling of colour-selected galaxy samples, and could dominate the error budget on all mass scales for both colours (Zentner et al. 2014).

The cause for the theoretical uncertainties in predictions of the halo mass and bias functions is three-fold: i) the Universe may have a non- $\Lambda \mathrm{CDM}$ cosmology (for halo statistics beyond $\Lambda \mathrm{CDM}$, see Bhattacharya et al. 2011, Cui et al. 2012a, Ichiki \& Takada 2012, Murray et al. 2013, Zhang et al. 2013, LoVerde 2014) or a different set of cosmological parameters (especially $\sigma_{8}$; see Planck Collaboration et al. 2015, for details on the tension between different probes) than the particular $\Lambda \mathrm{CDM}$ cosmology we adopt; ii) the predictions should be inaccurate due to the unaccounted-for baryonic effects (at 5 to 20 per cent level depending on the mass scale and the feedback models, see Cui et al. 2012b; Cusworth et al. 2014, Velliscig et al. 2014); and iii) the predictions may be poorly calibrated on the high mass end where the halos are rare (at the 5 per cent level for $M_{h}=10^{15} h^{-1} M_{\odot}$ at $z=0$, see Crocce et al. 2010. Watson et al. 2013; Bocquet et al. 2015). Among the three types of errors, the calibration errors affect few galaxies as the probability of any observed galaxies sitting in a halo with mass above $10^{15} h^{-1} M_{\odot}$ is extremely low, and are thus negligible in our analysis. Errors due to wrong cosmology, ignored baryonic effects, or the combination of both, change the halo mass and bias functions together in a coherent manner that can be roughly mimicked by changing $\Omega_{m}$ and/or $\sigma_{8}$ within the $\Lambda \mathrm{CDM}$ model. In particular, changing $\Omega_{m}$ at fixed $\sigma_{8}$ shifts the predictions along the halo mass axis almost uniformly (albeit with a minor change in the functional shapes due to the change in the power spectrum), while changing $\sigma_{8}$ at fixed $\Omega_{m}$ modifies the amplitude of the predictions more pro- 
gressively at higher halo mass (see figure 7 in $\mathrm{Zu}$ et al. 2014, for a pedagogical illustration). We explore the impact of i) and ii) by perturbing $\Omega_{m}$ and $\sigma_{8}$ using both theoretical arguments and mock tests below.

In our analysis on large scales, we are effectively using the halo bias vs. halo abundance relation compressed from the halo mass and bias functions, i.e., the bias of $M_{h}$-thresholded halo samples $b(\bar{n})$ as a function of the co-moving number density of that sample, $\bar{n}\left(>M_{h}\right)$. Due to the exponential decline of the abundance of massive halos, the theoretical uncertainties in this $b(\bar{n})-\bar{n}\left(>M_{h}\right)$ relation are only important at the high mass end, where the galaxy sample is primarily composed of massive BCGs with small satellite fraction. In the context of the peak background-split theory of biased clustering (Kaiser 1984, Cole \& Kaiser 1989. Mo et al. 1996. Sheth \& Tormen 1999), for very rare, very highly biased peaks, $b(\bar{n}) \propto \sigma_{8}^{-1}$, yielding $w_{p}(\bar{n}) \propto b^{2}(\bar{n}) \xi_{m m} \propto b^{2}(\bar{n}) \sigma_{8}^{2} \simeq$ constant, and $\Delta \Sigma \propto \Omega_{m} b(\bar{n}) \xi_{m m} \propto \Omega_{m} \sigma_{8}$, respectively. These two approximations hold even in the presence of baryons. Therefore, the clustering is barely affected by the changes in $\Omega_{m}$ or $\sigma_{8}$, while the lensing is linearly responsive to the change in the product of $\Omega_{m}$ and $\sigma_{8}$. Since the galaxy clustering measurements have a better overall $S / N$ than the g-g lensing measurements, a $10 \%$ change in $\Omega_{m} \sigma_{8}$ should produce a shift in the SHMR smaller than 0.06 dex in $M_{h}$ (if all of our constraints came from large scales). To confirm this, we re-run the analysis using two different combinations of $\left(\Omega_{m}, \sigma_{8}\right)=(0.26,0.80)$ and $(0.28,0.80)$. For the first case where we change $\sigma_{8}$ alone, the shift in the derived SHMR is indiscernible, whereas for the latter case where we increase the product of $\Omega_{m}$ and $\sigma_{8}$ by $12 \%$, the derived SHMR shifts to the lower halo mass by $\sim 0.08$ dex, corresponding to an increase in the stellar mass at $M_{h}=10^{13} h^{-1} M_{\odot}$ by only $\sim 0.03 \mathrm{dex}$, with little change in the parameters that control the satellite HOD.

The remaining sources of theoretical systematic errors, including the uncertainties in the mean halo mass-concentration relation $c_{\mathrm{dm}}\left(M_{h}\right)$ (we also ignore the dispersion in the halo concentration at fixed $M_{h}$ ), calibration in the scale-dependence of halo bias $\zeta(r)$, and the deviation of the cross-correlation coefficient $r_{\mathrm{cc}}(r)$ from unity, are expected to be much smaller than the four main errors discussed above. Summing all the systematic errors in quadrature, we would find the total systematic error comparable to or even dominate over the statistical uncertainties in the measurements. However, as pointed out in Coupon et al. (2015), each of these systematic errors affects the inference independently with different stellar mass and scale dependencies and we fit all of them jointly, the combined impact of these systematic errors should be sub-dominant compared to the statistical errors.

\section{STELLAR MASS CONTENT WITHIN HALOS}

In this section, we explore the implications of our inferred HOD parameters for the connection between stellar and halo mass.

\subsection{Stellar Mass Functions}

One of the most important consistency-checks for our fiducial iHOD model is to predict the parent SMFs at each redshift, and compare to the observed versions as well as the intrinsic SMFs estimated empirically, e.g., from the $V_{\max }$ method. As mentioned in Section 5.1 this check echoes the philosophy of the abundance matching technique, but in practice is carried out in reverse. In particular, the model uses the observed 2-point correlation function to infer the mapping from the stellar mass content, including the central and satellite galaxies, to the dark matter halos, and the mapping in turn gives an exact prediction (via Equation 17) for the parent SMF at each redshift - a "correlation" matching as opposed to "abundance" matching.

Figure 9 presents the results of this consistency check on SMFs at six different redshifts. For each redshift, the upper sub-panel compares the observed (brown histogram), $V_{\max }-$ estimated (thin black line), and our inferred parent (thick black line with 1- $\sigma$ uncertainty band) SMFs. The parent SMF, predicted from the best-fit $i$ HOD model at that redshift, is also decomposed into the central (red dashed) and satellite (blue dot-dashed) contributions, except for the $z=0.04$ panel where we instead show another measured SMF from Li \& White (2009) (shifted by 0.1 dex to remove the average bias between the two stellar mass estimators; see figure A1 in their paper). The central galaxies dominate the satellite galaxies in numbers across all stellar masses. The lower subpanel shows the ratios of the observed (brown line with Poisson errors) and estimated SMFs (thin black line) over the parent one, i.e., the observed fraction $f_{\text {obs }}$. The redshift evolution of the predicted parent SMFs is solely from the cosmic evolution of the halo mass function, and the Panter et al. (2007) SMF is the same in all panels. Although the stellar mass estimates from Panter et al. (2007) were independently derived from the SDSS DR3 spectroscopy, the SMF (also used for the abundance matching in M10) provides a good fit to the observed SMF using the MPA/JHU stellar masses, indicating little systematic offset between the two estimators. Overall, at $M_{*}>5 \times 10^{10} h^{-2} M_{\odot}$ where the completeness of SDSS is expected to be high, the predicted parent SMFs show remarkable consistency with the observed SMFs in both the shape and the amplitude at all redshifts. As mentioned in Section 5.1, the $i$ HOD analysis is completely agnostic to the total number density of the galaxy catalogue (i.e. the normalisation of the observed SMFs). Therefore, the agreement seen in Figure 9 demonstrates that, in order to match the clustering and lensing signal measured in SDSS, the placement of galaxies within the dark matter halos is uniquely determined so that the expected abundance of the galaxies at each stellar mass, translated from the halo mass function predicted by the $\Lambda \mathrm{CDM}$ Universe, automatically agrees with the observed galaxy SMFs in SDSS.

For the two lowest redshifts, the observed SMFs at the high mass end are known to be notably incomplete due to two photometric confusions: 1) to avoid saturation and excessive cross-talk in the spectrographs, some objects were rejected because they either have saturated centres (mostly bright active nuclei) or are blended with a saturated star; and 2) the image deblending software sometimes over-deblended bright galaxies with large angular extent (Strauss et al. 2002). A somewhat related issue is the over-subtraction of the sky background mentioned in Section 2.2. which results in suppressed flux estimates in bright galaxies. Therefore, limited to the $z<0.05$ galaxies, the SMF measured by Li \& White has a substantially lower amplitude at $M_{*}>3 \times 10^{10} h^{-2} M_{\odot}$ than the Panter et al's SMF, the observed SMFs beyond $z>0.08$, and the parent SMF predicted by our best-fit model.

We next consider the $f_{\text {obs }}$ inferred from the ratio of the $V_{\text {max }}$ estimated SMF to the predicted parent SMF in the lower sub-panels of Fig. 9. Starting with the low mass end, $f_{\text {obs }}$ is always above $60 \%$, and reaches $100 \%$ at above $6 \times 10^{10} h^{-2} M_{\odot}$ until $2 \times 10^{11} h^{-2} M_{\odot}$, beyond which point the $V_{\max }$ method is expected to underestimate the galaxy abundance due to the aforementioned photometric is- 


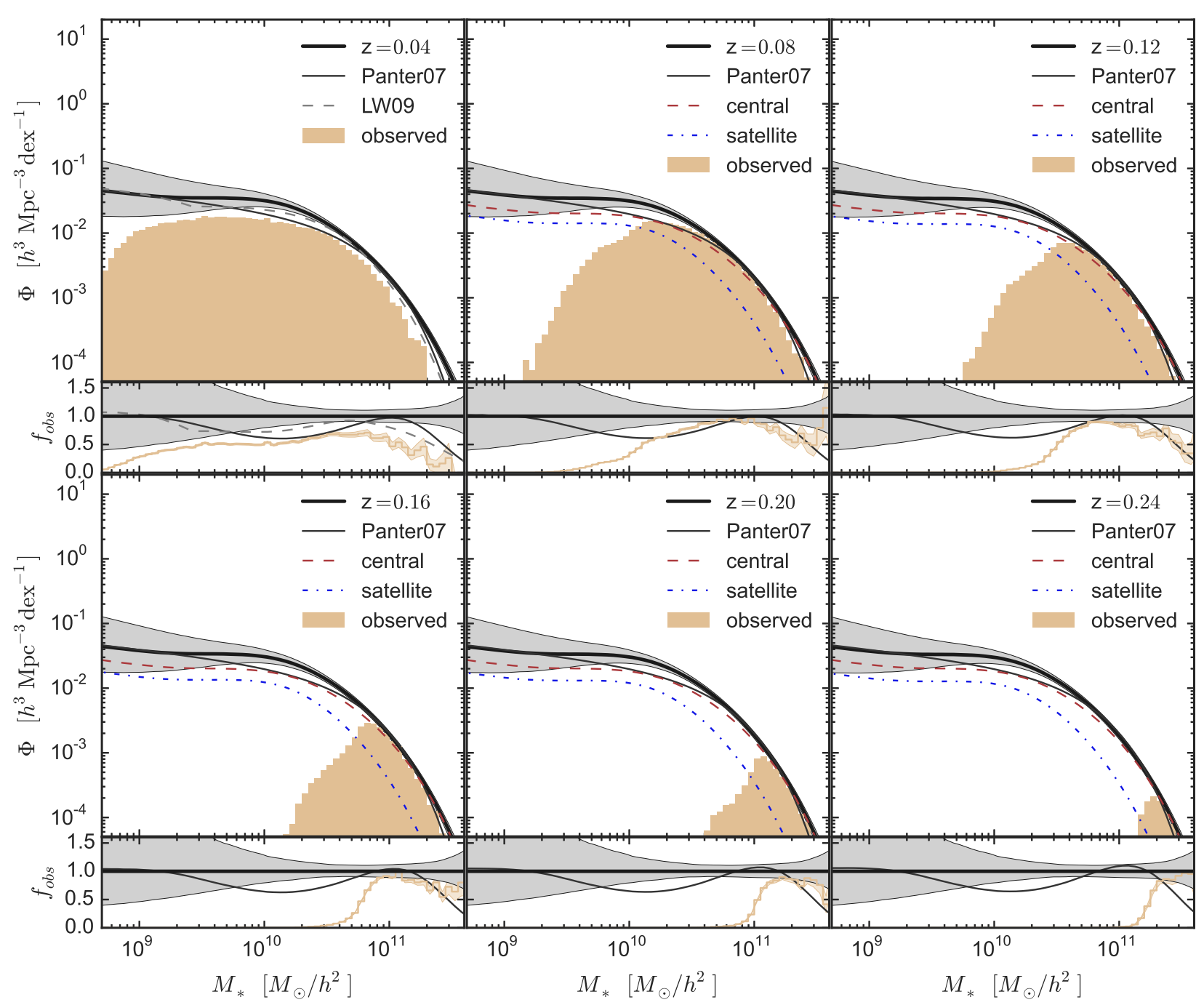

Figure 9. Comparison between the observed (brown histograms) and the parent (thick solid curves with $68 \%$ confidence range shown in gray bands) galaxy stellar mass functions predicted from the best-fit $i$ HOD model at six different redshifts. In each upper sub-panel, the thin solid curve is the estimated SMF of SDSS DR3 galaxies from Panter et al. (2007). For the $z=0.04$ panel, we show another SDSS SMF estimated by Li \& White (2009) from the $z<0.05$ galaxies as the thin dashed curve (after correction for the offset between the two stellar mass estimates); for the rest of the panels the predict parent SMF is decomposed into the contributions from the central and satellite galaxies, indicated by the red dashed and blue dot dashed lines, respectively. The lower sub-panels show the ratios of the Panter et al. (2007) (thin black) and the observed galaxy number density (brown) to the predicted SMF from our model. The redshift evolution of the predicted SMF in the model is coming solely from the cosmic evolution of the halo mass function.

sues. Meanwhile at the high mass end, the iHOD model predicts a much better match to the observed SMFs than the Panter et al's estimate does, reaching a constant $f_{\text {obs }}$ of $\sim 90 \%$, especially at the two highest redshifts where the photometric confusions that plague the lower redshifts are absent and the sample of very massive galaxies should be highly complete.

At the mass scale around $10^{10} h^{-2} M_{\odot}$, the $f_{\text {obs }}$ inferred from the $V_{\text {max }}$-estimated SMF exhibits a dip at $60 \%-70 \%$ level in the lower sub-panels of Figure 9 There are two main possible sources that could contribute to this dip, one theoretical and one observational. On the one hand, the dip could be a manifestation of the systematic uncertainty in the theoretical model. As seen from Figure 11 , this mass range marks the transition of galaxies from a predominantly blue, star-forming population below to a red, quiescent population above on the $M_{*}-z$ diagram. Given the strong correlation between galaxy colour and $M_{*} / L$, we expect that the ansatz we have made, i.e., assuming a weak dependence of $f_{\mathrm{obs}}\left(M_{*} \mid z\right)$ on the host halo mass, to be the most fragile at this transitional mass scale. For example, if the $M_{*} / L$ at fixed stellar mass is skewed higher in more massive halos (Taylor et al. 2015), the observed $10^{10} h^{-2} M_{\odot}$ galaxies would preferentially reside in host halos less massive than does a typical $10^{10} h^{-2} M_{\odot}$ galaxy in the Universe, and thus exhibit weaker clustering and g-g lensing signals than their stellar mass suggests. As a result of this masquerade effect, the $I$ HOD model would place them inside smaller halos, resulting the over-prediction of their abundances. It is worth noting that this theoretical systematic uncertainty is neither absent from the traditional HOD modelling, nor would it be eliminated by adding the SMF data as an extra constraint. On the other hand, the photometric issues that affect the targeting of bright galaxies would also impact this intermediate mass range, rendering the starforming galaxy population underrepresented in the MGS at the low 
redshift. The selection effect is easily discernible in Figure $1 \mathrm{a}$ : at $M_{*} \sim 10^{10} h^{-2} M_{\odot}$, the galaxies at redshifts lower than 0.04 have a redder average $g-r$ colour than those at higher redshifts. Since the majority of the detected galaxies with $M_{*} \sim 10^{10} h^{-2} M_{\odot}$ comes from $z<0.08$, they carry heavy weight (large values of $1 / V_{\max }$ ) in the Panter et al's and Li \& White's SMFs. Therefore, the underrepresentation of these galaxies at low redshift translates to potentially significant underestimate of their abundances. In reality it is more likely that both the theoretical and observational sources contribute to the dip at some level, and the true parent SMF should lie somewhere between the Panter et al's and our predicted curves.

\subsection{Centrals: Stellar Mass to Halo Mass Relation}

The most crucial piece for mapping the stellar content back to dark matter halos is the SHMR of the central galaxies. The top panel of Figure 10 compares the SHMR and its logarithmic scatter derived from our fiducial $i$ HOD constraints (black solid line with gray band) to that inferred from several methods, including abundance matching. To highlight the shrinkage in scatter at high halo mass predicted by our fiducial constraint, we also show two curves that delineate the boundaries of the constant scatter band with $\eta=0$. To avoid clutter, here we only show the scatter for the SHMR from our constraint.

Let us first compare to the constraint from Moster et al. (2010) (red dashed), which employed the same cosmology as our analysis and matches the halo abundance to the SDSS SMF measured by Panter et al. (2007), albeit with a different functional form for the SHMR and a fixed scatter. The two mean SHMRs show remarkable agreement over $\lg M_{h} \sim[12-14]$, or $M_{*} \sim 2 \times 10^{10}$ $2 \times 10^{11} h^{-2} M_{\odot}$, where the data are the most constraining and the completeness is the highest. Above this mass range, the Moster et al's relation predicts higher stellar mass for central galaxies than our mean SHMR for a given halo mass, i.e., a steeper SHMR. Considering the lower amplitude of the $V_{\max }$-estimated SMF employed by the Moster et al. (2010) analysis for abundance matching, naively one might expect that the observed massive galaxies have to be pushed to reside in progressively rarer and more massive halos, making their SHMR shallower instead. However, due to the exponential decline of the halo mass function in this regime, the SHMR is much more sensitive to the change in scatter than the small change in the detection rate. This discrepancy on the high mass end is mainly caused by the smaller scatter assumed in the Moster et al. (2010) analysis $\left(\sigma_{\ln M_{*}}=0.345\right.$, or 0.15 in dex) compared to that inferred by our model $\left(\sigma_{\ln M_{*}} \sim 0.40\right.$ at $\left.M_{h}=10^{15} h^{-1} M_{\odot}\right)$. Their smaller scatter allows much fewer low-mass halos to be considered as hosts for the massive galaxies, thus requiring a steeper slope in order to match the galaxy abundance.

The Behroozi et al. (2010) analysis derives the constraints on the SHMR by abundance matching to the SDSS SMF measured by $\mathrm{Li} \&$ White (2009) at $z<0.05$. They carefully accounted for the effect of scatter in the SHMR by varying both the scatters in the true stellar mass at fixed halo mass (intrinsic scatter) and in the observed stellar estimate at fixed true stellar mass (measurement scatter). For the intrinsic scatter, a log-normal prior centred at 0.16 dex with a width of 0.04 dex is placed (i.e., $0.37 \pm 0.09$ in natural $\log$ ), and for the measurement scatter a fixed value of $0.07 \mathrm{dex}$ is applied for the SDSS data. After abundance matching, the posterior constraint on the intrinsic scatter is $0.15_{-0.02}^{+0.04} \mathrm{dex}$, consistent with their input prior. However, as clearly shown by the top left panel of Figure 9 the Li \& White (2009) SMF is underestimated at the high mass end, thus shifting the mean SHMR horizontally toward higher
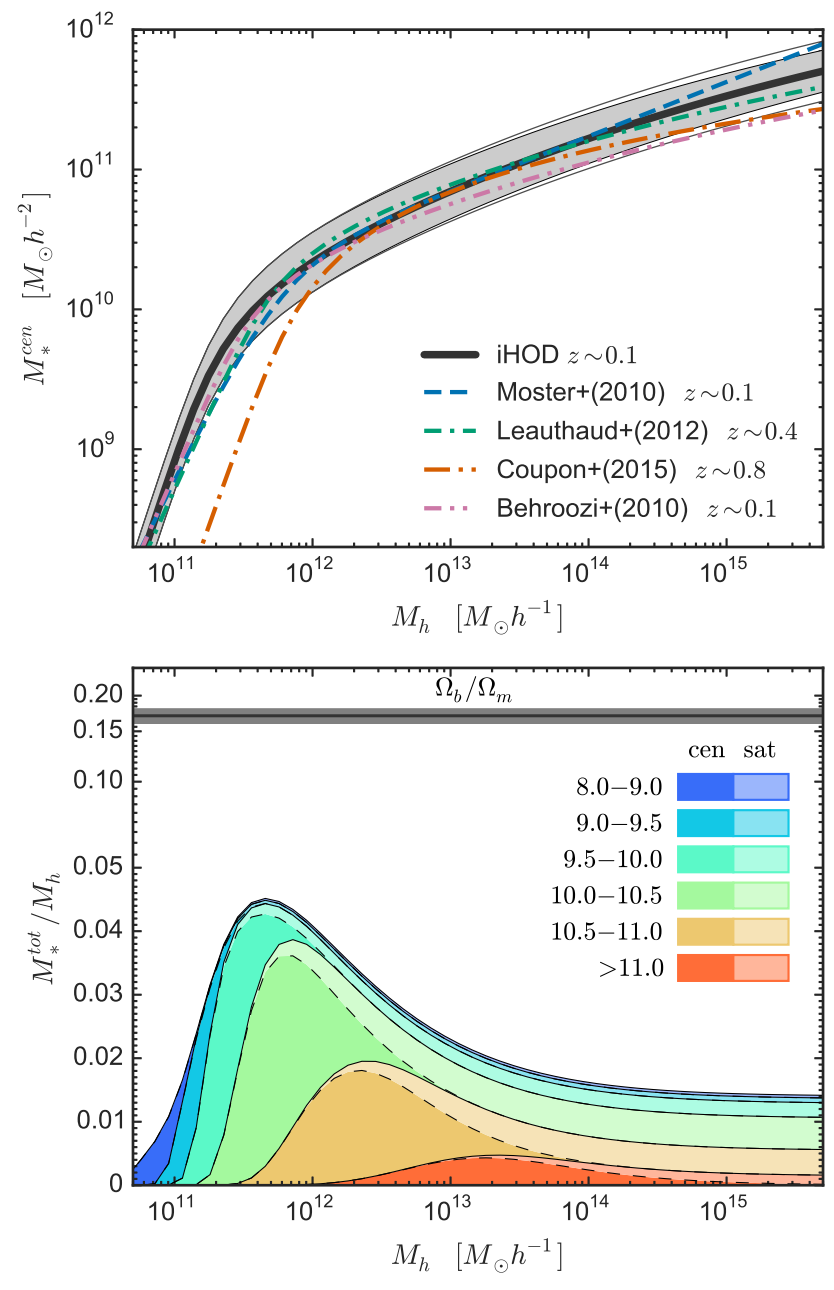

Figure 10. Top panel: Comparison between the SHMRs inferred from abundance-matching based methods and our best-fit iHOD model. The gray band shows the logarithmic scatter predicted by the best-fit model, while the two enveloping thin curves represent the range of constant scatter with $\eta=0$. Among the four external constraints used for comparison, the Moster et al. (2010) and the Behroozi et al. (2010) results are based on abundance-matching, while the other two are derived from fitting the clustering, lensing, and SMF jointly. Bottom panel: The total stellar mass to halo mass ratio as a function of halo mass. Coloured layers show the contribution from different stellar mass bins while separate portions of the same coloured layer indicate the relative contribution from centrals and satellites within each stellar mass bin. The horizontal band represents the cosmic baryon to dark matter ratio in the Universe. Note that the y-axis is logarithmic above 0.05 .

halo mass. Behroozi et al. (2010) also adopts a slightly higher $\sigma_{8}$ than M10 and our study, which predicts more massive halos and further pushes the slope of the mean SHMR shallower than our constraint.

Unlike abundance matching, our fiducial constraint is able to break the degeneracy between the scatter and the slope of the mean SHMR on the high mass end without assuming any external priors. The leverage comes from the unique constraining power of combining clustering and lensing. On large scales, the galaxy clustering signal is proportional to $b_{g}^{2} \xi_{m m}$, while the g-g lensing scales with $\Omega_{m} b_{g} \xi_{m m}$. On small scales, $w_{p}$ is less a probe of the SHMR than $\Delta \Sigma$, which directly measures the average halo mass of individual galaxy samples. For a fixed cosmology, simultaneous fit to 
$w_{p}$ and $\Delta \Sigma$ effectively constrains the clustering bias as a function of halo mass, for which $\Lambda \mathrm{CDM}$ predicts a relatively steep slope on the high mass end (see the seventh and eighth columns of Table 1 . However, as shown by the open contours in the $\delta-\sigma_{\ln M_{*}}$ panel in Figure 7, the $C H O D$ constraint stills shows substantial degeneracy between the slope parameter $\delta$ and the scatter, due to the inadequate $S / N$ in the measurements of $w_{p}$ and $\Delta \Sigma$. Thanks to the $84 \%$ more galaxies used in the $i$ HOD analysis, our fiducial analysis derives much tighter constraint with minimum residual degeneracy (filled contours).

The L12 result shown in Figure 10 was derived from a slightly higher redshift range $(0.22<z<0.48)$ than the MGS used by us. Since we have adopted a similar model framework and parameterization proposed in L12, the analysis of Leauthaud et al. (2012b) is very similar to the $\mathrm{CHOD}$ analysis in this paper. However, their analysis adds the measured SMF as part of the constraining data set beside angular clustering and g-g lensing. In this regard, although allowing the scatter to vary freely, the constraint in Leauthaud et al. (2012b) is somewhat more related to the AM-based methods, because the smaller uncertainties in SMF easily dominate the likelihood of the fit. A straightforward comparison with the mean SHMR of Leauthaud et al. (2012b) is complicated by the differences in the redshift range of the galaxy samples and the stellar mass estimators (although the COSMOS SMF below $z=0.48$ agrees very well with that from Panter et al. 2007; see figure 5 of L12), but the two constraints appear to be qualitatively consistent with each other above the characteristic halo mass. The Coupon et al. (2015) constraint is very similar to L12, derived from a joint lensing, clustering, and abundance analysis, but for galaxies at a much higher redshift $(z \sim 0.8)$ and more massive than $10^{10} h^{-2} M_{\odot}$. Thus, the deviation between the Coupon et al. (2015) SHMR and ours at the low mass end can be attributed to the evolution of galaxy population from $z=0.8$ to $z=0.1$. At the high end however, we do not see the steepening of the Coupon et al. (2015) SHMR at $z \sim 0.8$, as hinted by the L12 constraints from $z \sim 0.88$ (see figure 11 in L12). The L12 and the Coupon et al. (2015) analyses also constrained the scatter in the SHMR, yielding $\sigma_{\ln M_{*}} \simeq 0.474$ (i.e., $0.206 \mathrm{dex}$ ) and $\sigma_{\ln M_{*}} \simeq 0.506$ (i.e., $0.22 \mathrm{dex}$; at $M_{h} \simeq 10^{12} h^{-1} M_{\odot}$ ), respectively, in excellent agreement with our constraint of $0.50_{-0.03}^{+0.04}$. This level of agreement is not necessarily expected, given that the scatter includes both intrinsic scatter and measurement uncertainty, the latter of which could differ for the three different samples studied in these papers.

Below the characteristic halo mass, our mean SHMR has a slightly higher amplitude than all the other three SHMRs, implying higher stellar-to-halo mass ratios for the low stellar mass galaxies. This difference echoes the discrepancy seen in the comparison between the parent and measured SMFs at the intermediate and low stellar mass scales. However, all those curves are still consistent with one another within the statistical uncertainties in the inferred mean SHMRs below $M_{h} \sim 10^{12} h^{-1} M_{\odot}$ (compare the difference to, e.g., the uncertainty band in the left panel of Figure 8).

The efficiency of stellar mass assembly at the centre of halos, characterised by the central stellar-to-halo mass ratio $M_{*}^{\text {cen }} / M_{h}$, rises sharply as a function of halo mass at the low mass scale, probably due to the increasing difficulty for the stellar feedback to drive cold gas out of the steepening gravitational potential wells of halos (Hopkins et al. 2012). The growth of the central galaxies shifts down into a much lower gear beyond the characteristic mass $\sim 10^{12} h^{-1} M_{\odot}$, decreasing $M_{*}^{\text {cen }} / M_{h}$ from its peak $\sim 0.04$ at $M_{h} \sim 10^{12} h^{-1} M_{\odot}$ to below 0.001 at $M_{h} \sim 10^{15} h^{-1} M_{\odot}$. AGN feedback is believed to be one of the major culprits that quench the star formation in those central galaxies within high mass halos. However, as shown by Figure 3 the satellite "cloud" also begins to emerge within halos above $10^{12} h^{-1} M_{\odot}$, and a good indicator for the efficiency of stellar mass assembly has to account for the stellar mass of satellites. To this end, the bottom panel of Figure 10 presents a more complete picture by showing the total stellar mass assembly efficiency, $M_{*}^{\text {tot }} / M_{h}$ (assuming $h=0.72$ ), integrated over all the predicted parent galaxies above $10^{8} h^{-2} M_{\odot}$. We also divide the total efficiency into contributions from six different stellar mass bins marked in the legend. Within the layer of each stellar mass bin, we further split the contribution into two components, the central (shaded colour) and the satellite (tinted colour), separated by a dashed line. For comparison, the horizontal line on top indicates the universal baryon-to-matter mass ratio. As expected, the total efficiency is dominated by the central galaxies until its peak at around $M_{h} \sim 10^{12} h^{-1} M_{\odot}$, then it levels off and asymptote to a constant value $\sim 0.015$ for galaxy groups and clusters, where the satellite contribution dominates. This asymptotic value of the total stellar mass fraction is consistent with observational studies by stacking galaxy catalogues or images inside samples of optical and/or X-ray clusters (Budzynski et al. 2014, Bahcall \& Kulier 2014). Galaxies with $M_{*}$ around $10^{10} h^{-2} M_{\odot}$ dominate the total stellar mass content within halos of mass above $10^{12} h^{-1} M_{\odot}$.

The near-constant total stellar-to-halo mass ratio in group and cluster-size halos is very intriguing. By comparing the integrated stellar mass to the stacked weak lensing signal at different radii within the MaxBCG clusters, Bahcall \& Kulier (2014) found that the total mass of clusters can be largely accounted for by the total dark matter mass associated with all the subhalos that host the satellite galaxies. Therefore, when the fractional contribution from the central galaxy is negligible,

$\left.\frac{\sum M_{*}}{M_{h}}\right|_{M_{h}} \simeq\left[\int\left(\left.\frac{d N_{\mathrm{sat}}}{d \lg M_{*}}\right|_{M_{h}}\right) \frac{M_{*}}{M_{h}^{\mathrm{sub}}} d \lg M_{*}\right] / N_{\mathrm{sat}}\left(M_{h}\right)$,

where $\left.\left(d N_{\text {sat }} / d \lg M_{*}\right)\right|_{M_{h}}$ is the satellite stellar mass function conditioned at halo mass $M_{h}$, and $M_{h}^{\text {sub }}$ is the subhalo mass. If the satellite stellar-to-subhalo mass relation $M_{*} / M_{h}^{\text {sub }}$ were to roughly follow the SHMR of the centrals, as assumed by our subhalo lensing model and the SHAM methods, the aggregate of the satellite galaxy population inside each halo is likely to be a strong function of the halo mass when $d N_{\text {sat }} / d \lg M_{*}$ differs from halo to halo. In order for any two halos with different masses to share the same total stellar-to-halo mass ratio, the stellar mass functions within the two halos have to be somewhat self-similar - all $d N_{\text {sat }} / d \lg M_{*}$ have the same shape near the knee of the SMF where most of the stellar mass is stored, with their amplitudes $N_{\text {sat }}$ linearly proportional to the halo mass. To test this hypothesis, we explore the conditional SMFs of satellites below in Section 7.3

The SHMR, specifying the mean logarithmic stellar mass of central galaxies at fixed halo mass, is a convenient choice from a theoretical modelling point of view, but observationally what is often measured is the opposite, i.e., the mean log-halo mass or halo mass at given stellar mass. For instance, as the predecessor of the study in this paper, Mandelbaum et al. (2006) measured the average log-halo masses for a set of early and late-type galaxy stellar mass samples in the SDSS, via the traditional HOD modelling of their g$\mathrm{g}$ lensing signals. To compare to the result from Mandelbaum et al. (2006) and other similar studies in the literature, we compute the distribution of the halo mass at fixed stellar mass as

$$
p\left(M_{h} \mid M_{*}^{\text {cen }}\right)=\frac{p\left(M_{*}^{\text {cen }} \mid M_{h}\right) p\left(M_{h}\right)}{p\left(M_{*}^{\text {cen }}\right)},
$$




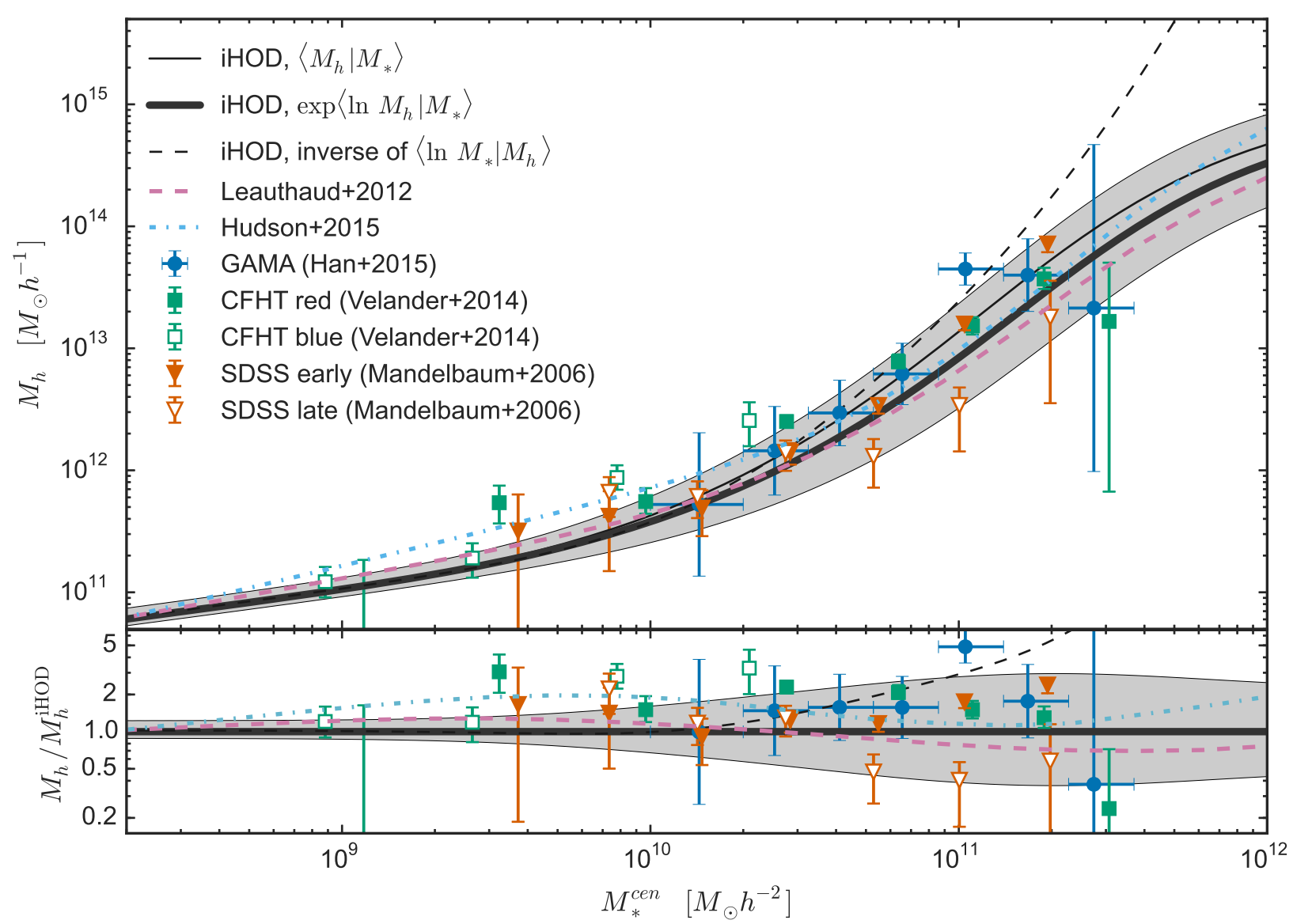

Figure 11. Comparison between the halo-to-stellar mass relations inferred from the best-fit iHOD model and that from various other weak lensing measurements. Top panel: the dash black curve is just the inverse function of the best-fit SHMR, i.e., the same black curve in the top panel of Figure 10 but with $\mathrm{x}$ - and $y$-axis swapped. The thick black curve with a gray band indicate the mean and scatter (not statistical uncertainty on the mean) of the distribution of logarithmic halo mass at fixed stellar mass, while the thin black curve shows the mean halo mass at fixed stellar mass. The magenta dashed and cyan dot-dashed curves are the results from L12 using COSMOS and Hudson et al. (2015) using CFHTLenS, while the blue circles are from the maximum-likelihood weak lensing analysis of GAMA galaxies. The green and the red squares are the average weak lensing masses measured for CFHTLenS and SDSS galaxies, respectively, and within each survey the relations are constrained for red/early-type and blue/late-type galaxies separately using nearly identical HOD prescriptions. All the uncertainties shown for the weak lensing mass measurements are 1- $\sigma$. Bottom panel: Similar to the top panel, but showing the ratio of each quantity over the prediction by the best-fit $i$ HOD model.

where $p\left(M_{*}^{\text {cen }} \mid M_{h}\right)$ is the SHMR of central galaxies (similar to Equation 21 and $p\left(M_{h}\right)$ is the halo mass function normalised by the total number density of halos, while $p\left(M_{*}^{\text {cen }}\right)$ is proportional to the SMF of central galaxies, calculated from

$$
p\left(M_{*}^{\mathrm{cen}}\right)=\int_{0}^{+\infty} p\left(M_{*}^{\mathrm{cen}} \mid M_{h}\right) p\left(M_{h}\right) \mathrm{d} M_{h} .
$$

The halo-to-stellar mass relation, $p\left(M_{h} \mid M_{*}^{\text {cen }}\right)$, computed from the best-fit SHMR and its scatter shown in the top panel of Figure 10 via Equations (47) and (48), is represented in Figure 11 in two forms, the mean log-halo mass at fixed stellar mass (thick black solid curve),

$$
\left\langle\ln M_{h} \mid M_{*}\right\rangle=\int p\left(M_{h} \mid M_{*}^{\mathrm{cen}}\right) \ln M_{h} \mathrm{~d} M_{h},
$$

and the mean halo mass at fixed stellar mass (thin black solid curve),

$$
\left\langle M_{h} \mid M_{*}\right\rangle=\int p\left(M_{h} \mid M_{*}^{\text {cen }}\right) M_{h} \mathrm{~d} M_{h},
$$

respectively. The difference between the two means are small at the low stellar mass end but grows to 0.1 dex at high masses, and the gray band indicates the 1- $\sigma$ logarithmic scatter about the mean relation. As seen in Figure 10 the original SHMR flattens on the high mass end, so the log-scatter in halo mass at fixed stellar mass is much larger than on the low stellar mass end. The $1-\sigma$ error on the mean relation (not shown here, but see Figure 8 ) is comparable to the scatter at the lower stellar mass end, but much smaller than the scatter above $M_{*} \sim 10^{10} h^{-2} M_{\odot}$. Also shown on Figure 11 is the black dashed curve from Equation (19) using the best-fit parameters, i.e., the same thick black curve shown in Figure 10 except that the $\mathrm{x}$ - and $\mathrm{y}$-axises are swapped. The difference between the black dashed and the two black solid curves can easily exceed 0.5 dex for high stellar mass samples, and reaches almost 1 dex for galaxies with $M_{*}>5 \times 10^{11} h^{-2} M_{\odot}$. Therefore, caution should be exercised when trying to compare the theoretical SHMR with the direct observational estimates of halo masses for any galaxy samples selected above $M_{*}=10^{10} h^{-2} M_{\odot}$. The magenta dashed and the cyan dot-dashed curves in Figure 11 are the halo-to-stellar mass relations inferred from the L12 (COSMOS) and the Hudson et al. (2015) (CFHTLenS) analyses, respectively, showing great consis- 

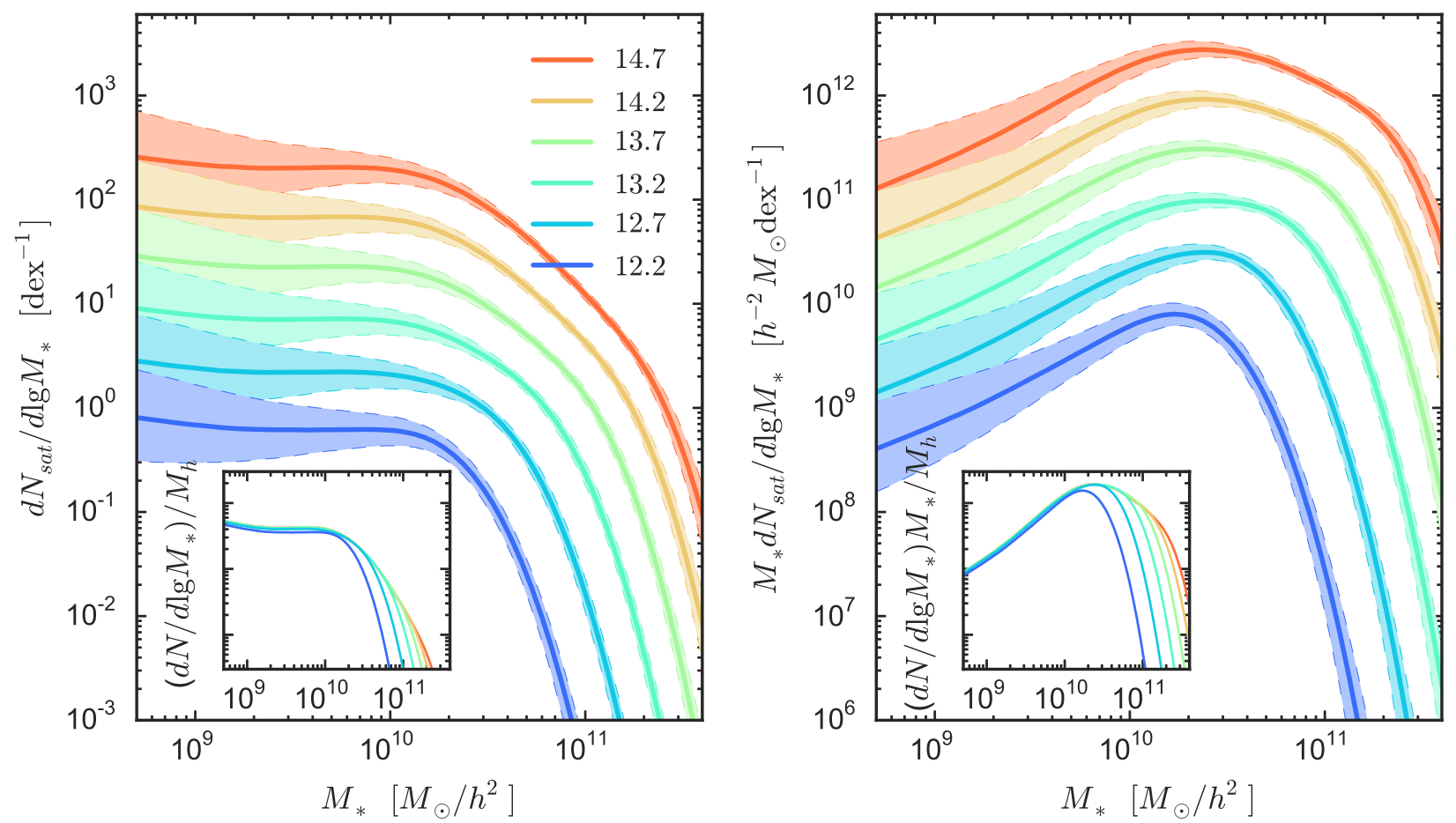

Figure 12. Left panel: Satellite stellar mass function $d N_{\text {sat }} / d M_{*}$ conditioned at six different halo masses marked on the top right. The shaded regions indicate the $68 \%$ uncertainties derived from the iHOD constraint. The inset panel shows the same set of curves, with each amplitude re-scaled by the corresponding halo mass, highlighting the departure of the conditional SMF from the Schechter functional form in high mass halos. Right panel: Similar to the left panel, but for $M_{*} d N_{\mathrm{sat}} / d M_{*}$, the fractional contribution to the total stellar-to-halo mass ratio per dex in $M_{*}$. The satellite galaxies with stellar mass around a few times $10^{10} h^{-2} M_{\odot}$ contribute the most to the total stellar mass content in each halo.

tency between independent constraints from different surveys. The circles and the squares indicate the average halo masses inferred from individual galaxy stellar mass samples using the weak lensing measurements in GAMA (Han et al. 2015) and CFHTLenS (Velander et al. 2014), respectively. The lenses used in the Han et al. (2015) analysis were central galaxies of groups and clusters that are almost volume-limited, while for the latter two, the Velander et al. (2014) constraint was separately derived for colour-segregated samples that are flux-limited. Despite the differences in the methods and data sets, our relation is largely consistent with both sets of measurements. The triangles are the measurements from the earlier SDSS DR4 galaxies (Mandelbaum et al.2006), also split into sub-samples of early- and late-type galaxies. Our analysis is a direct update from the Mandelbaum et al. (2006) study, using SDSS DR7 (vs. DR4) galaxies, updated MPA/JHU stellar mass catalogue, improved photometric redshifts for the source sample, additional measurements (galaxy clustering), and a more sophisticated HOD model, and the two constraints are fully consistent with each other.

Finally, to facilitate the comparison between our constraint and observational studies that measure the mean (log)halo mass of individual galaxy samples, we provide an analytic fitting formula to the best-fit halo-to-stellar mass relation shown in Figure 11 (computed from the fiducial i HOD MCMC chain via Equation 49,

$$
\begin{aligned}
\left\langle\lg M_{h} \mid M_{*}^{\text {cen }}\right\rangle & =4.41\left[1+\exp \left(-1.82\left(\lg M_{*}^{\text {cen }}-11.18\right)\right)\right]^{-1} \\
& +11.12 \sin \left(-0.12\left(\lg M_{*}^{\text {cen }}-23.37\right)\right)
\end{aligned}
$$

where $M_{h}$ and $M_{*}^{\text {cen }}$ are in units of $h^{-1} M_{\odot}$ and $h^{-2} M_{\odot}$, respectively. The above fitting formula is accurate to within $0.15 \%$ across the entire stellar mass range above $3 \times 10^{8} h^{-2} M_{\odot}$.

\subsection{Satellites: Conditional Stellar Mass Function}

Instead of imposing some fixed functional form (e.g., the Schechter function) for the conditional stellar mass function (CSMF), we compute the satellite CSMFs as the derivatives of the satellite HODs at fixed halo mass. This model flexibility allows for a more thorough exploration of the shapes of the satellite CSMFs, especially any potential mass-dependent deviations from the Schechter function at both the low and high stellar mass ends. The left panel of Figure 12 shows the satellite CSMFs inferred for halos of six different masses marked on the legend, with each shaded band indicating the $68 \%$ uncertainty from the $i$ HOD constraint. For low mass halos, the form of the CSMFs resemble the Schechter function with a flattening low $M_{*}$ portion and a sharp exponential cutoff at high $M_{*}$, but it starts to exhibit an extra power-law portion at intermediate $M_{*}$ for halos more massive than $10^{13} h^{-1} M_{\odot}$. The inset panel shows the six CSMFs scaled by their corresponding halo mass. All the scaled CSMFs would land on top of one another if the satellite population inside halos follows an exact homology sequence. Since $\alpha_{\text {sat }}$ is strongly constrained to be around unity $\left(\alpha_{\text {sat }}=\right.$ $\left.1.000_{-0.02}^{+0.03}\right)$, the number of satellite galaxies above any stellar mass threshold scales linearly with halo mass above $M_{\text {cut }}$ (see Equation (22), leading to a self-similar behaviour across the low $M_{*}$ range $\left(<10^{10} h^{-2} M_{\odot}\right)$. At high stellar mass range, however, the homology is broken and an excess population of galaxies of successively higher stellar mass begin to emerge as satellites - they used to be the central galaxies of small halos that were later accreted by their current-day, more massive host halos - a physical picture from the hierarchical structure formation paradigm of the $\Lambda \mathrm{CDM}$ Universe, recovered by the prediction of our fiducial 
model, a purely statistical framework that describes the clustering and lensing of galaxies.

To understand the asymptotic behaviour of the total stellarto-halo mass ratio mentioned in Section 7.2 we show the stellar mass-weighted CSMFs, $M_{*} d N_{\text {sat }} / d \lg M_{*}$, in the right panel of Figure 12 The peaks in those curves confirm the finding from the bottom panel of Figure 10 that galaxies of $M_{*} \sim 2 \times 10^{10} h^{-2} M_{\odot}$ contribute the most amount of stellar mass per dex in $M_{*}$ to each halo. Again, in the inset panel we scale each quantity by the corresponding halo mass, showing the fractional contribution to the total stellar-to-halo mass ratio per dex in $M_{*}$ for each halo mass. The asymptotic behaviour of the total stellar mass fraction can be mainly attributed to two factors: 1) the self-similarity of satellites galaxies below $10^{11} h^{-2} M_{\odot}$ ensures that the total ratio integrated up to $M_{*} \sim 10^{11} h^{-2} M_{\odot}$ is the same for all halos above $5 \times 10^{13} h^{-1} M_{\odot}$; and 2) the satellite galaxies above $10^{11} h^{-2} M_{\odot}$ contribute negligibly to the total ratio, so any increase in halo mass brings little change to the total stellar-to-halo mass ratio.

\section{CONCLUSIONS}

We have developed a novel extension to the HOD framework namely the IHOD model — to solve for the mapping between the observed stellar mass distribution and the underlying dark matter halos, via modelling the projected galaxy auto-correlation function $w_{p}$ and the g-g lensing $\Delta \Sigma$ signals. In particular, the iHOD model has two main features:

- It is able to predict $w_{p}$ and $\Delta \Sigma$ for all the galaxies above the mixture limit (i.e., the minimum stellar mass for the quiescent galaxies to be detected above the flux limit). This flexibility allows us to include $\simeq 84 \%$ more galaxies than the traditional HOD method, which substantially improves the $S / N$ of the $w_{p}$ and $\Delta \Sigma$ measurements (by $10 \%$ to almost a factor of two depending on stellar mass)

- It takes into account the volume incompleteness of galaxy stellar mass samples in a statistically consistent way, eliminating the need to assume completeness or parameterize the redshiftdependent selection functions.

The crucial input to the $I$ HOD model is the shape of the observed SMF at each redshift, which is employed by the $i$ HOD model to construct an HOD for the galaxies at that redshift slice using $p\left(M_{*}, M_{h}\right)$, the 2D joint probability density distribution of a galaxy with stellar mass $M_{*}$ sitting in a halo of mass $M_{h}$. The clustering and lensing signals of any galaxy sample can then be calculated by combining the signals predicted from the HODs of the individual redshift slices.

The 2D distribution $p\left(M_{*}, M_{h}\right)$ has two components, the mean and scatter of the SHMR for the central galaxies and the global HOD for the satellite galaxies. We adopt a similar parameterization for the two components as L11, but allow the powerlaw slope of the satellite HOD $(\alpha)$ and the concentration ratio between the satellite and dark matter profiles $\left(f_{c}\right)$ to vary freely during the constraint. Furthermore, we also re-calibrate the subhalo lensing model in our analysis against the state-of-the-art hydrodynamic simulation MassiveBlack-II. Thanks to the greatly improved $S / N$ in the $w_{p}$ and $\Delta \Sigma$ measurements, our fiducial iHOD analysis not only breaks the degeneracy between the slope and the scatter of the SHMR, therefore placing stringent constraint on the link between the dark halos and their central galaxies, but also derives the conditional SMF of satellite galaxies as a function of halo mass.
The inferred SHMR is in good agreement with constraints from the abundance matching methods and the joint clustering, lensing, and galaxy abundance analyses using the L11 framework. For the SMF of satellite galaxies at fixed halo mass, the best-fit model predicts a departure from the Schechter function in massive groups and clusters, probably a result of mergers and accretion that convert central galaxies of small halos into satellites of more massive systems. It will be interesting to compare the conditional SMFs of satellites statistically derived from the $i$ HOD model, which allows the satellites to have a different SHMR than the central galaxies, to that inferred from abundance matching, which directly assigns satellite stellar masses to subhalos in the simulations assuming the same SHMR as the central galaxies.

In principle, the $i$ HOD model uses only the shape of the observed galaxy SMF as input and is agnostic of the SMF amplitude - using half of the galaxies randomly drawn from the original catalogue would yield the same best-fit model parameters, albeit with larger uncertainties. However, the parent SMF predicted from the best-fit $i$ HOD model agrees remarkably well with the observed SMF in both the shape and the amplitude. Since the amplitude of the predicted SMF is translated from the normalisation of the halo mass function predicted by the $\Lambda \mathrm{CDM}$ cosmology, this agreement is highly non-trivial, demonstrating the efficacy of the i HOD model — in order to match the clustering and lensing signal measured in SDSS, the mapping between the galaxy stellar content and the dark matter halos is uniquely determined so that the expected galaxy abundance at each $M_{*}$, calculated by summing galaxies at that $M_{*}$ over all the dark matter halos, automatically gives the observed galaxy SMF in SDSS.

In future work, we will extend the $i \mathrm{HOD}$ model to provide constraints on the quenching mechanisms that transform galaxies from star-forming to quiescent. The most straightforward approach is to apply the same analysis to just the quenched population (i.e., the galaxies with $g-r>0.8$ above the mixture limit), and compare the inferred $p_{\text {red }}\left(M_{*}, M_{h}\right)$ to the best-fit $p\left(M_{*}, M_{h}\right)$ in this paper to predict the quenched fraction as a function of $M_{*}$ and $M_{h}$ (see e.g., Zehavi et al. 2005, Zehavi et al. 2011, for HOD studies of galaxies segregated by colour). Alternatively, one can do a joint fit for the red and blue galaxies simultaneously, assuming an ad hoc functional form for the quenched fraction (see, e.g., Tinker et al. 2013. Rodríguez-Puebla et al. 2015). However, as mentioned in Section 6.3 the galaxy assembly bias needs to be carefully treated or marginalised over to obtain a robust constraint when modelling colour-selected samples.

Going beyond the goal of understanding galaxy formation, HOD modelling of galaxy populations is likely to have a role in cosmological analyses of future large imaging surveys that seek to constrain dark energy using weak lensing, such as the Large Synoptic Survey Telescope (LSST; LSST Science Collaborations \& LSST Project 2009), Euclid (Laureijs et al.2011), and WFIRST (Spergel et al.2015). While cosmological weak lensing was originally identified as a very clean probe of dark energy due to its sensitivity to matter fluctuations, more recent work has identified two major theoretical uncertainties: the effect of baryons on the matter power spectrum, and the intrinsic alignments of galaxy shapes that violate the assumption that any coherent galaxy alignments are due to weak lensing. Leading proposals for the mitigation of these theoretical uncertainties include halo modelling of the galaxy-shear crosscorrelation (galaxy-galaxy lensing) in combination with galaxy clustering and cosmic shear, instead of using cosmic shear alone (e.g., Schneider \& Bridle 2010, Semboloni et al. 2011, 2013, Zentner et al. 2013). Thus, we anticipate that the iHOD model will be 
an important contribution to such efforts, given that it will enable the use of a larger galaxy sample in understanding these important contaminants to cosmic shear surveys, and thus enable the use of tighter priors on the nuisance parameters for baryonic effects and/or intrinsic alignments when extracting cosmological information from the cosmic shear signal.

As a general yet powerful statistical formalism, the $i \mathrm{HOD}$ model can be easily applied to galaxies at higher redshifts, such as the existing datasets from the COSMOS survey (Scoville et al. 2007) used by L12, CFHTLenS (Heymans et al.2012), the recently finished Baryon Oscillation Spectroscopic Survey (BOSS; Eisenstein et al. 2011; Dawson et al. 2013) and its near-term higher redshift successor eBOSS, and the deeper surveys planned for future facilities such as the Dark Energy Spectroscopic Instrument (DESI; Levi et al. 2013), the Subaru Prime Focus Spectrograph (Takada et al.2014), Euclid, and WFIRST. Galaxies at higher redshifts, such as luminous red galaxies (LRGs; Eisenstein et al. 2001; Anderson et al. 2012) and emission line galaxies (Comparat et al. 2015), are usually targeted with some complicated colour and flux cuts, so the galaxy samples are always volume-incomplete. For instance, Hoshino et al. (2015) found that the average number of LRG-type central galaxies in the massive halos does not asymptotically reach unity, contradictory to the assumption in traditional HOD models (Parejko et al. 2013). Therefore, the iHOD model would be an especially valuable tool in constraining the link between galaxies and dark matter halos at high redshifts.

\section{ACKNOWLEDGEMENTS}

We thank Neta Bahcall, David Weinberg, and Zheng Zheng for helpful discussions, and Jiaxin Han for providing his weak lensing mass measurements. We thank Alexie Leauthaud for carefully reading an earlier version of the manuscript and for giving detailed comments and suggestions that have greatly improved the manuscript. We also thank the MassiveBlack-II team for providing the simulated galaxy catalogue and the dark matter particle data. Y.Z. acknowledges the hospitality of the CCAPP at the Ohio State University where he enjoyed a fruitful discussion with participants of the "Assembly Bias and the Galaxy-Halo Relation" Workshop. R.M. and Y.Z. are supported by the Department of Energy Early Career Program, and the Sloan Fellowship program.

\section{REFERENCES}

Abazajian K. N. et al., 2009, ApJS, 182, 543

Anderson L. et al., 2012, MNRAS, 427, 3435

Bahcall N. A., Kulier A., 2014, MNRAS, 439, 2505

Baldauf T., Smith R. E., Seljak U., Mandelbaum R., 2010, Phys. Rev. D, 81,063531

Baldry I. K., Balogh M. L., Bower R. G., Glazebrook K., Nichol R. C., Bamford S. P., Budavari T., 2006, MNRAS, 373, 469

Bardeen J. M., Bond J. R., Kaiser N., Szalay A. S., 1986, ApJ, 304, 15

Baugh C. M., 2006, Reports on Progress in Physics, 69, 3101

Behroozi P. S., Conroy C., Wechsler R. H., 2010, ApJ, 717, 379

Bell E. F., de Jong R. S., 2001, ApJ, 550, 212

Benson A. J., 2010, Physics Reports, 495, 33

Berlind A. A., Kazin E., Blanton M. R., Pueblas S., Scoccimarro R., Hogg D. W., 2006, astro-ph/0610524

Berlind A. A., Weinberg D. H., 2002, ApJ, 575, 587

Bernardi M., Meert A., Sheth R. K., Vikram V., Huertas-Company M., Mei S., Shankar F., 2013, MNRAS, 436, 697
Bhattacharya S., Heitmann K., White M., Lukić Z., Wagner C., Habib S., 2011, ApJ, 732, 122

Blanton M. R., Berlind A. A., 2007, ApJ, 664, 791

Blanton M. R. et al., 2005, AJ, 129, 2562

Blazek J., Mandelbaum R., Seljak U., Nakajima R., 2012, J. Cosmology Astropart. Phys., 5, 41

Bocquet S., Saro A., Dolag K., Mohr J. J., 2015, arXiv:1502.07357

Bond J. R., Cole S., Efstathiou G., Kaiser N., 1991, ApJ, 379, 440

Booth C. M., Schaye J., 2009, MNRAS, 398, 53

Bower R. G., Benson A. J., Malbon R., Helly J. C., Frenk C. S., Baugh

C. M., Cole S., Lacey C. G., 2006, MNRAS, 370, 645

Bruzual G., Charlot S., 2003, MNRAS, 344, 1000

Budzynski J. M., Koposov S. E., McCarthy I. G., Belokurov V., 2014, MNRAS, 437, 1362

Budzynski J. M., Koposov S. E., McCarthy I. G., McGee S. L., Belokurov V., 2012, MNRAS, 423, 104

Cacciato M., van den Bosch F. C., More S., Li R., Mo H. J., Yang X., 2009, MNRAS, 394, 929

Cacciato M., van den Bosch F. C., More S., Mo H., Yang X., 2013, MNRAS, 430, 767

Cappellari M. et al., 2012, Nature, 484, 485

Cen R., Ostriker J. P., 1999, ApJ, 514, 1

Chabrier G., 2003, PASP, 115, 763

Cole S., Kaiser N., 1989, MNRAS, 237, 1127

Comparat J. et al., 2015, A\&A, 575, A40

Conroy C., 2013, ARA\&A, 51, 393

Conroy C., van Dokkum P. G., 2012, ApJ, 760, 71

Conroy C., Wechsler R. H., Kravtsov A. V., 2006, ApJ, 647, 201

Cooray A., Sheth R., 2002, Phys. Rep., 372, 1

Coupon J. et al., 2015, MNRAS, 449, 1352

Cowie L. L., Songaila A., Hu E. M., Cohen J. G., 1996, AJ, 112, 839

Crocce M., Fosalba P., Castander F. J., Gaztañaga E., 2010, MNRAS, 403, 1353

Croft R. A. C., Matteo T. D., Khandai N., Springel V., Jana A., Gardner J. P., 2012, MNRAS, 425, 2766

Croton D. J., Gao L., White S. D. M., 2007, MNRAS, 374, 1303

Cui W., Baldi M., Borgani S., 2012a, MNRAS, 424, 993

Cui W., Borgani S., Dolag K., Murante G., Tornatore L., 2012b, MNRAS, 423, 2279

Cusworth S. J., Kay S. T., Battye R. A., Thomas P. A., 2014, MNRAS, 439, 2485

Dalal N., White M., Bond J. R., Shirokov A., 2008, ApJ, 687, 12

Davé R., Finlator K., Oppenheimer B. D., 2012, MNRAS, 421, 98

Dawson K. S. et al., 2013, AJ, 145, 10

De Lucia G., Blaizot J., 2007, MNRAS, 375, 2

De Lucia G., Springel V., White S. D. M., Croton D., Kauffmann G., 2006, MNRAS, 366, 499

Di Matteo T., Springel V., Hernquist L., 2005, Nature, 433, 604

Dutton A. A., Macciò A. V., 2014, MNRAS, 441, 3359

Einasto J., 1965, Trudy Astrofizicheskogo Instituta Alma-Ata, 5, 87

Eisenstein D. J. et al., 2001, AJ, 122, 2267

Eisenstein D. J. et al., 2011, AJ, 142, 72

Faltenbacher A., White S. D. M., 2010, ApJ, 708, 469

Faucher-Giguère C.-A., Kereš D., Ma C.-P., 2011, MNRAS, 417, 2982

Feldmann R. et al., 2006, MNRAS, 372, 565

Ferreras I., La Barbera F., de la Rosa I. G., Vazdekis A., de Carvalho R. R.,

Falcón-Barroso J., Ricciardelli E., 2013, MNRAS, 429, L15

Fontanot F., De Lucia G., Monaco P., Somerville R. S., Santini P., 2009, MNRAS, 397, 1776

Foreman-Mackey D., Hogg D. W., Lang D., Goodman J., 2013, PASP, 125,306

Fukugita M., Hogan C. J., Peebles P. J. E., 1998, ApJ, 503, 518

Fukugita M., Ichikawa T., Gunn J. E., Doi M., Shimasaku K., Schneider D. P., 1996, AJ, 111, 1748

Gallazzi A., Bell E. F., 2009, ApJS, 185, 253

Gao, L., Navarro, J. F., Cole, S., et al. 2008, MNRAS, 387, 536

Gao L., Springel V., White S. D. M., 2005, MNRAS, 363, L66

Gao L., White S. D. M., 2007, MNRAS, 377, L5 
Geller M. J., Huchra J. P., 1989, Science, 246, 897

George M. R. et al., 2012, ApJ, 757, 2

George M. R. et al., 2011, ApJ, 742, 125

Gunn J. E. et al., 1998, AJ, 116, 3040

Gunn J. E. et al., 2006, AJ, 131, 2332

Guo H., Zehavi I., Zheng Z., 2012, ApJ, 756, 127

Guo H. et al., 2014, MNRAS, 441, 2398

Guo Q. et al., 2011, MNRAS, 413, 101

Guo Q., White S., Li C., Boylan-Kolchin M., 2010, MNRAS, 404, 1111

Guzik J., Seljak U., 2001, MNRAS, 321, 439

Guzik J., Seljak U., 2002, MNRAS, 335, 311

Hahn O., Porciani C., Carollo C. M., Dekel A., 2007, MNRAS, 375, 489

Hamilton A. J. S., Tegmark M., 2004, MNRAS, 349, 115

Han J. et al., 2015, MNRAS, 446, 1356

Hansen S. M., Sheldon E. S., Wechsler R. H., Koester B. P., 2009, ApJ, 699, 1333

Harker G., Cole S., Helly J., Frenk C., Jenkins A., 2006, MNRAS, 367, 1039

Hearin A. P., Watson D. F., 2013, MNRAS, 435, 1313

Hearin A. P., Watson D. F., van den Bosch F. C., 2014, arXiv:1404.6524

Hernquist L., Katz N., 1989, ApJS, 70, 419

Heymans C. et al., 2012, MNRAS, 427, 146

Hirata C., Seljak U., 2003, MNRAS, 343, 459

Hopkins P. F., Quataert E., Murray N., 2012, MNRAS, 421, 3522

Hoshino H. et al., 2015, arXiv:1503.05200

Hudson M. J. et al., 2015, MNRAS, 447, 298

Ichiki K., Takada M., 2012, Phys. Rev. D, 85, 063521

Jing Y. P., Mo H. J., Börner G., 1998, ApJ, 494, 1

Jing Y. P., Suto Y., Mo H. J., 2007, ApJ, 657, 664

Kaiser N., 1984, ApJ, 284, L9

Kang X., Jing Y. P., Mo H. J., Börner G., 2005, ApJ, 631, 21

Katz N., Weinberg D. H., Hernquist L., 1996, ApJS, 105, 19

Kauffmann G. et al., 2003, MNRAS, 341, 33

Kereš D., Katz N., Weinberg D. H., Davé R., 2005, MNRAS, 363, 2

Kerscher M., Szapudi I., Szalay A. S., 2000, The Astrophysical Journal Letters, 535, L13

Khandai N., Di Matteo T., Croft R., Wilkins S. M., Feng Y., Tucker E., DeGraf C., Liu M., 2014, ArXiv e-prints, 1402, 888

Kravtsov A. V., Berlind A. A., Wechsler R. H., Klypin A. A., Gottlöber S., Allgood B., Primack J. R., 2004, ApJ, 609, 35

Kulier A., Ostriker J. P., 2015, arXiv:1503.07533

Landy S. D., Szalay A. S., 1993, ApJ, 412, 64

Lang D., Hogg D. W., Mierle K., Blanton M., Roweis S., 2010, AJ, 139, 1782

Laporte C. F. P., White S. D. M., 2014, arXiv:1409.1924

Laureijs R. et al., 2011, arXiv:1110.3193

Leauthaud A. et al., 2012a, ApJ, 746, 95

Leauthaud A., Tinker J., Behroozi P. S., Busha M. T., Wechsler R. H., 2011, ApJ, 738, 45

Leauthaud A. et al., 2012b, ApJ, 744, 159

Levi M. et al., 2013, arXiv:1308.0847

Li C., White S. D. M., 2009, MNRAS, 398, 2177

Li R. et al., 2014, MNRAS, 438, 2864

Li Y., Mo H. J., Gao L., 2008, MNRAS, 389, 1419

LoVerde M., 2014, Phys. Rev. D, 90, 083530

LSST Science Collaborations, LSST Project, 2009, ArXiv e-prints (0912.0201), http://www.lsst.org/lsst/scibook

Ma C.-P., Fry J. N., 2000, ApJ, 543, 503

Madau P., Dickinson M., 2014, ARA\&A, 52, 415

Mandelbaum R., Hirata C. M., Leauthaud A., Massey R. J., Rhodes J., 2012, MNRAS, 420, 1518

Mandelbaum R. et al., 2005, MNRAS, 361, 1287

Mandelbaum R., Seljak U., Cool R. J., Blanton M., Hirata C. M., Brinkmann J., 2006, MNRAS, 372, 758

Mandelbaum R., Seljak U., Kauffmann G., Hirata C. M., Brinkmann J., 2006, MNRAS, 368, 715

Mandelbaum R., Slosar A., Baldauf T., Seljak U., Hirata C. M., Nakajima

R., Reyes R., Smith R. E., 2013, MNRAS, 432, 1544
Mandelbaum R., Tasitsiomi A., Seljak U., Kravtsov A. V., Wechsler R. H., 2005, MNRAS, 362, 1451

McGaugh S. S., Schombert J. M., de Blok W. J. G., Zagursky M. J., 2010, ApJ, 708, L14

Miyatake H. et al., 2013, arXiv:1311.1480

Mo H. J., Jing Y. P., White S. D. M., 1996, MNRAS, 282, 1096

More S., Miyatake H., Mandelbaum R., Takada M., Spergel D., Brown-

stein J., Schneider D. P., 2014, arXiv:1407.1856

Moster B. P., Somerville R. S., Maulbetsch C., van den Bosch F. C., Macciò A. V., Naab T., Oser L., 2010, ApJ, 710, 903

Murray S. G., Power C., Robotham A. S. G., 2013, MNRAS, 434, L61

Nakajima R., Mandelbaum R., Seljak U., Cohn J. D., Reyes R., Cool R., 2012, MNRAS, 420, 3240

Navarro J. F., Frenk C. S., White S. D. M., 1997, ApJ, 490, 493

Newman A. B., Ellis R. S., Treu T., 2015, arXiv:1503.05282

Newman A. B., Treu T., Ellis R. S., Sand D. J., Nipoti C., Richard J., Jullo E., 2013, ApJ, 765, 24

Norberg P., Baugh C. M., Gaztañaga E., Croton D. J., 2009, MNRAS, 396, 19

Norman M. L., Bryan G. L., 1999, in Astrophysics and Space Science Library, Vol. 240, Numerical Astrophysics, Miyama S. M., Tomisaka K., Hanawa T., eds., p. 19

Oppenheimer B. D., Davé R., 2006, MNRAS, 373, 1265

Oppenheimer B. D., Davé R., 2008, MNRAS, 387, 577

O'Shea B. W., Bryan G., Bordner J., Norman M. L., Abel T., Harkness R., Kritsuk A., 2004, astro-ph/0403044

Padmanabhan N. et al., 2008, ApJ, 674, 1217

Panter B., Jimenez R., Heavens A. F., Charlot S., 2007, MNRAS, 378 , 1550

Parejko J. K. et al., 2013, MNRAS, 429, 98

Peacock J. A., Smith R. E., 2000, MNRAS, 318, 1144

Peebles P. J. E., Hauser M. G., 1974, ApJS, 28, 19

Peng Y.-j., Lilly S. J., Renzini A., Carollo M., 2012, ApJ, 757, 4

Planck Collaboration et al., 2015, arXiv:1502.01589

Prada F., Klypin A. A., Cuesta A. J., Betancort-Rijo J. E., Primack J., 2012, MNRAS, 3206

Press W. H., Schechter P., 1974, ApJ, 187, 425

Putman M. E., Peek J. E. G., Joung M. R., 2012, ARA\&A, 50, 491

Reddick R. M., Wechsler R. H., Tinker J. L., Behroozi P. S., 2013, ApJ, 771,30

Reyes R., Mandelbaum R., Gunn J. E., Nakajima R., Seljak U., Hirata C. M., 2012, MNRAS, 425, 2610

Rodríguez-Puebla A., Avila-Reese V., Yang X., Foucaud S., Drory N., Jing Y. P., 2015, ApJ, 799, 130

Salim S. et al., 2007, ApJS, 173, 267

Schmidt F., Rozo E., Dodelson S., Hui L., Sheldon E., 2009, Phys. Rev. Lett., 103, 051301

Schneider M. D., Bridle S., 2010, MNRAS, 402, 2127

Scoccimarro R., Sheth R. K., Hui L., Jain B., 2001, ApJ, 546, 20

Scoville N. et al., 2007, ApJS, 172, 1

Seljak U., 2000, MNRAS, 318, 203

Semboloni E., Hoekstra H., Schaye J., 2013, MNRAS, 434, 148

Semboloni E., Hoekstra H., Schaye J., van Daalen M. P., McCarthy I. G., 2011, MNRAS, 417, 2020

Shankar F. et al., 2014, The Astrophysical Journal Letters, 797, L27

Shankar F., Lapi A., Salucci P., De Zotti G., Danese L., 2006, ApJ, 643, 14

Sheldon E. S. et al., 2004, AJ, 127, 2544

Sheth R. K., Tormen G., 1999, MNRAS, 308, 119

Sheth R. K., Tormen G., 2004, MNRAS, 350, 1385

Shull J. M., Smith B. D., Danforth C. W., 2012, ApJ, 759, 23

Simet M., Mandelbaum R., 2015, MNRAS, 449, 1259

Singh S., Mandelbaum R., More S., 2014, arXiv:1411.1755

Smith R. E. et al., 2003, MNRAS, 341, 1311-1332

Somerville R. S., Hopkins P. F., Cox T. J., Robertson B. E., Hernquist L., 2008, MNRAS, 391, 481

Spergel D. et al., 2015, arXiv:1503.03757

Springel V. et al., 2005, Nature, 435, 629 
Strauss M. A. et al., 2002, AJ, 124, 1810

Swanson M. E. C., Tegmark M., Hamilton A. J. S., Hill J. C., 2008, MNRAS, 387, 1391

Takada M. et al., 2014, PASJ, 66, 1

Takahashi R., Sato M., Nishimichi T., Taruya A., Oguri M., 2012, ApJ, 761,152

Taylor E. N. et al., 2015, MNRAS, 446, 2144

Teyssier R., 2002, A\&A, 385, 337

Tinker J., Kravtsov A. V., Klypin A., Abazajian K., Warren M., Yepes G., Gottlöber S., Holz D. E., 2008, ApJ, 688, 709-728

Tinker J. L., Leauthaud A., Bundy K., George M. R., Behroozi P., Massey R., Rhodes J., Wechsler R. H., 2013, ApJ, 778, 93

Tinker J. L., Robertson B. E., Kravtsov A. V., Klypin A., Warren M. S., Yepes G., Gottlöber S., 2010, ApJ, 724, 878

Tinker J. L., Weinberg D. H., Zheng Z., Zehavi I., 2005, ApJ, 631, 41

Vale A., Ostriker J. P., 2004, MNRAS, 353, 189

van Daalen M. P., Schaye J., 2015, arXiv:1501.05950

van den Bosch F. C., More S., Cacciato M., Mo H., Yang X., 2013, MNRAS, 430, 725

van den Bosch F. C., Tormen G., Giocoli C., 2005, MNRAS, 359, 1029

van den Bosch F. C. et al., 2007, MNRAS, 376, 841

van Dokkum P. G., Conroy C., 2010, Nature, 468, 940

Velander M. et al., 2014, MNRAS, 437, 2111

Velliscig M., van Daalen M. P., Schaye J., McCarthy I. G., Cacciato M.,

Le Brun A. M. C., Dalla Vecchia C., 2014, MNRAS, 442, 2641

Vogelsberger M., Genel S., Sijacki D., Torrey P., Springel V., Hernquist L., 2013, MNRAS, 436, 3031

Vogelsberger M. et al., 2014, Nature, 509, 177

von der Linden A., Best P. N., Kauffmann G., White S. D. M., 2007, MNRAS, 379, 867

Wang H. Y., Mo H. J., Jing Y. P., 2007, MNRAS, 375, 633

Wang L., Weinmann S. M., De Lucia G., Yang X., 2013, MNRAS, 433, 515

Wang W., Sales L. V., Henriques B. M. B., White S. D. M., 2014, MNRAS, 442,1363

Watson D. F., Berlind A. A., McBride C. K., Hogg D. W., Jiang T., 2012, ApJ, 749, 83

Watson W. A., Iliev I. T., D’Aloisio A., Knebe A., Shapiro P. R., Yepes G., 2013, MNRAS, 433, 1230

Wechsler R. H., Zentner A. R., Bullock J. S., Kravtsov A. V., Allgood B., 2006, ApJ, 652, 71

Weinberg D. H., Davé R., Katz N., Hernquist L., 2004, ApJ, 601, 1

Yang X., Mo H. J., van den Bosch F. C., 2003, MNRAS, 339, 1057

Yang X., Mo H. J., van den Bosch F. C., 2009, ApJ, 695, 900

Yang X., Mo H. J., van den Bosch F. C., Pasquali A., Li C., Barden M., 2007, ApJ, 671, 153

Yoo J., Seljak U., 2012, Phys. Rev. D, 86, 083504

Yoo J., Tinker J. L., Weinberg D. H., Zheng Z., Katz N., Davé R., 2006, ApJ, 652, 26

York D. G. et al., 2000, AJ, 120, 1579

Zehavi I. et al., 2011, ApJ, 736, 59

Zehavi I. et al., 2005, ApJ, 630, 1

Zentner A. R., Hearin A. P., van den Bosch F. C., 2014, MNRAS, 443, 3044

Zentner A. R., Semboloni E., Dodelson S., Eifler T., Krause E., Hearin A. P., 2013, Phys. Rev. D, 87, 043509

Zhang Y., Zhang P., Yang X., Cui W., 2013, Phys. Rev. D, 87, 023521

Zhao D. H., Jing Y. P., Mo H. J., Börner G., 2009, ApJ, 707, 354-369

Zheng Z. et al., 2005, ApJ, 633, 791

Zheng Z., Weinberg D. H., 2007, ApJ, 659, 1

Zhu G., Zheng Z., Lin W. P., Jing Y. P., Kang X., Gao L., 2006, ApJ, 639, L5

Zu Y., Weinberg D. H., Rozo E., Sheldon E. S., Tinker J. L., Becker M. R., 2014, MNRAS, 439, 1628

Zu Y., Zheng Z., Zhu G., Jing Y. P., 2008, ApJ, 686, 41

\section{APPENDIX A: SMALL-SCALE LENSING CUTOFFS}

In this appendix, we describe our choice of minimum scale for modelling of the lensing signals. Our estimator for $\Delta \Sigma$, Eq. (6), is designed such that our signal includes a correction for the zero shear contributed by "source" galaxies that are at the lens redshift. This correction is often written separately as a "boost factor" (e.g., Sheldon et al. 2004, Mandelbaum et al. 2005),

$$
B\left(r_{p}\right)=\frac{\sum_{l s} w_{l s}\left(r_{p}\right)}{\sum_{r s} w_{r s}\left(r_{p}\right)},
$$

where the summed weights are over lens-source and random lenssource pairs. $B\left(r_{p}\right)=1$ if there are no galaxies associated with the lens that are included as sources and if lensing magnification is negligible. Generally, since both clustering and magnification are large at small $r_{p}, B\left(r_{p}\right)$ is a monotonically decreasing function.

As shown in Mandelbaum et al. (2005), small-scale systematics due to deblending and sky subtraction can appear as an increase in $B\left(r_{p}\right)$ on the smallest scales, followed by the expected decrease on scales where those systematics no longer operate. In the decreasing region, we know that source detection is no longer $100 \%$ effective, but what we do not know is the actual level of inefficiency, since $B\left(r_{p}\right)$ gives a single constraint on two completely generate quantities (amount of contamination by physically-associated galaxies, and inefficiency in the detection of both real sources and physically-associated ones). To properly correct for physicallyassociated sources, we would need to know both of these quantities. We would also have to know how the shear estimates and photo- $z$ estimates of the sources in those regions may have been modified due to the systematic that is causing difficulties with source detection. Due to the lack of sufficient information to model the signal on scales where $B\left(r_{p}\right)$ indicates small-scale systematics, we do not plot or attempt to model $\Delta \Sigma$ there. The quoted minimum scales used for the lensing modelling comes from this consideration.

There are two additional systematics that could, in principle, operate on small scales above our minimum cutoff. The first systematic is the intrinsic alignment of galaxy shapes, pointing coherently in the radial direction with respect to the lens. While intrinsic alignments of bright red galaxies are well-established in the literature (e.g., most recently, Singh et al. 2014), attempts to measure any radial alignments of faint galaxies in mixed blue and red source populations with respect to the positions of nearby bright galaxies have thus far only resulted in null detections (Blazek et al. 2012 with relatively tight upper limits. Hence we do not consider this to be an important systematic for this work.

Lensing magnification has also been considered as a systematic since, if present, it means that our normalization by $\sum_{r s} w_{r s}$ to correct for physically-associated sources is incorrect. As discussed in Mandelbaum et al. (2005) and Schmidt et al. (2009), the amount of magnification for a flux- and size-selected sample depends on both the slope of the number counts near the flux limit and the slope of the apparent size distribution near its limit. Moreover, it depends on the slopes of these distributions weighted by whatever per-object weights are used for the lensing analysis. Simet \& Mandelbaum (2015) calculated the appropriate slopes for the source catalogue used in this paper, and based on the numbers in their table 1 , the ratio of observed number counts with magnification to the un-magnified number counts is very near $1\left(n_{\mathrm{obs}} / n \approx 1-0.03 \kappa\right)$. Hence, even though the best-fitting model for our eight stellar mass samples have convergences that range from 0.0014 to 0.063 at $0.1 h^{-1} \mathrm{Mpc}$, the maximum effect of magnification on the observed counts and therefore the size of the systematic error in the boost 
factor and lensing calibration at that scale is -0.2 per cent. We therefore neglect this source of error. 